\title{
Extensões cindidas por ideais nilpotentes
}

\author{
Heily Wagner \\ DisSERTAÇÃo APRESENTADA \\ $\mathrm{AO}$ \\ Instituto de Matemática e Estatística \\ DA \\ Universidade DE SÃo PAUlO \\ PARA \\ OBTENÇÃO DO TÍTULO \\ $\mathrm{DE}$ \\ Mestre em CiênCIAS \\ Área de Concentração: Matemática \\ Orientador: Prof. Dr. Flávio Ulhoa Coelho
}

Durante o desenvolvimento deste trabalho o autor recebeu auxílio financeiro do CNPq

São Paulo, junho de 2008 


\section{Extensões cindidas por ideais nilpotentes}

Este exemplar corresponde à redação final da dissertação devidamente corrigida e defendida por Heily Wagner e aprovada pela Comissão Julgadora.

Banca Examinadora:

- Prof. Dr. Flávio Ulhoa Coelho (orientador) - IME-USP.

- Prof. Dr. Edson Ribeiro Alvares - UFPR.

- Prof. Dr. Clézio Aparecido Braga - UNIOESTE-PR. 


\section{Resumo}

Consideremos $A$ e $B$ duas álgebras de Artin tais que $B$ é uma extensão cindida de $A$ pelo ideal $Q$, onde $Q$ é um ideal nilpotente de $B$. Estudamos algumas propriedades homológicas das categorias $\bmod A$ e $\bmod B$, tais como dimensão projetiva e injetiva. A partir disso mostramos que se $B$ pertence a uma das seguintes classes: hereditária, laura, fracamente shod, shod, quase inclinada, colada à esquerda, colada à direita ou disfarçada; então $A$ pertence a mesma classe. Além disso, restringindo nosso estudo para álgebras de dimensão finita sobre um corpo algebricamente fechado, comparamos as respectivas aljavas ordinárias, bem como suas apresentações. Finalmente, após caracterizarmos o ideal $Q$, exibimos alguns exemplos de extensões no contexto de álgebras de caminhos com relações, que mostram que $A$ pode ser de uma das classes citadas sem que $B$ o seja.

Palavras-chave: extensões cindidas, representações de álgebras, dimensões homológicas. 


\begin{abstract}
Let $A$ and $B$ be two Artin algebras such that $B$ is a split-by-nilpotent extension of $A$ by $Q$, were $Q$ is a nilpotent ideal of $B$. We study some homological properties of the categories mod $A$ and $\bmod B$ such that the projetive and the injetive dimensions of their objects. Using this we show that if $B$ belongs to one of this classes: hereditary, laura, weakly shod, shod, quasi-tilted, left glued, right glued or concealed; then $A$ belongs to same class. Moreover restricting our study to finite dimensional algebras over algebraically closed fields, we compare the ordinary quivers and presentations of the corresponding algebras. Finally, after giving a characterization of ideal $Q$ as above, we exhibit some exemples of split extensions in the context of path algebras bounded by relations, which shows that $A$ can be one of the above cited algebras without $B$ so.
\end{abstract}

Keywords: split extensions, algebras representation, homological dimensions. 


\section{Sumário}

$\begin{array}{ll}\text { Introdução } & 1\end{array}$

1 Preliminares $\quad 5$

1.1 Categorias e Funtores . . . . . . . . . . . . . . . . . . . 5

1.2 Álgebras e Módulos . . . . . . . . . . . . . . . . . . . . . . . . 9

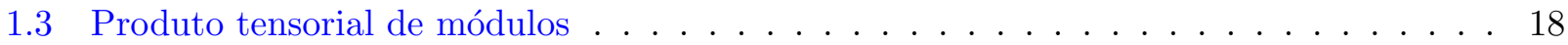

1.4 Álgebra de Caminhos . . . . . . . . . . . . . . . . . . . 21

2 Extensão cindida por nilpotente $\quad 27$

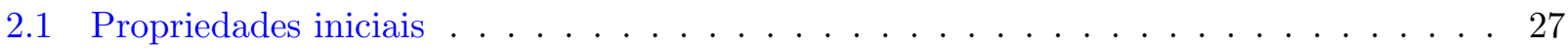

2.2 Categorias $\bmod A$ e $\bmod B \ldots \ldots \ldots \ldots \ldots \ldots$

2.3 Projetivos, injetivos e conexidade . . . . . . . . . . . . . . . . 34

3 Aljavas das extensões $\quad 39$

4 Propriedades homológicas herdadas $\quad 51$

4.1 Introdução . . . . . . . . . . . . . . . . . . . . . . . 51

4.2 Propriedades homológicas em $\bmod A$ e em $\bmod B \ldots \ldots$. . . . . . . . . . . 59

4.2.1 Álgebra hereditária e álgebra shod. . . . . . . . . . . . . . . . . 67 
$\begin{array}{lll}5 & \text { Parte direita e parte esquerda } & 69\end{array}$

5.1 Parte direita e parte esquerda da categoria de módulos . . . . . . . . . . . . . . . 69

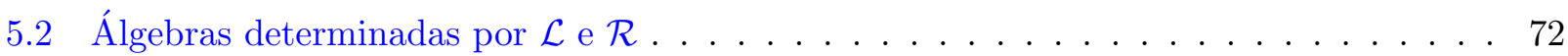

5.2 .1 Álgebra laura. . . . . . . . . . . . . . . . . . . . . 72

5.2 .2 Álgebras coladas à direita e à esquerda. . . . . . . . . . . . . . . . . . . 73

5.2 .3 Álgebra fracamente shod. . . . . . . . . . . . . . . . . . 74

5.2 .4 Álgebra shod . . . . . . . . . . . . . . . . . . . . . 75

5.2 .5 Álgebra quase inclinada. . . . . . . . . . . . . . . . . . . . . . . 75

5.2 .6 Álgebra disfarçada. . . . . . . . . . . . . . . . . . . . . 76

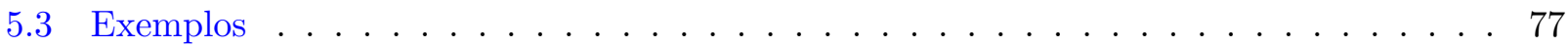

$\begin{array}{lr}\text { A Aljava de Auslander-Reiten } & 85\end{array}$

$\begin{array}{lr}\text { Referências Bibliográficas } & 89\end{array}$

$\begin{array}{ll}\text { Índice Remissivo } & 91\end{array}$ 


\section{Introdução}

A álgebra $B$ é dita uma extensão da álgebra $A$ se existir um epimorfismo de álgebras $\pi: B \rightarrow A$. Também podemos dizer que o par $(B, \pi)$ é uma extensão de $A$.

Este conceito aparece na literatura, por exemplo, em On the cohomology groups of an associative algebra (1945 - Annals of Mathematics 46, 58-67) de G. Hochschild, onde encontramos a chamada extensão singular, que é quando (Nuc $\pi)^{2}=0$ e a chamada extensão "segregate ", que é quando $B \cong A \oplus$ Nuc $\pi$. Neste trabalho, Hochschild trata (sem dar nome) de extensões "segregate "cujo núcleo (Nuc $\pi$ ) é um ideal nilpotente.

No livro Homological algebra (1956 - Princeton University Press) de H. Cartan e S. Eilenberg, uma extensão de álgebra $\pi: B \rightarrow A$ é dita inessencial quando $\pi$ tiver inverso à direita, isto é, se existir um morfismo de álgebras $\sigma: A \rightarrow B$ tal que $\pi \sigma=i d_{A}$. Atualmente dizemos que o epimorfismo cinde, ou ainda, que a extensão é cindida. Além disso podemos escrever $B \cong A \oplus$ Nuc $\pi$ (soma de A-módulos).

Nos trabalhos da última década, em especial [6], [7], [9] e [10] encontramos o termo que aqui será estudado: extensão cindida por ideal nilpotente.

Vamos estudar nesse trabalho características comuns entre duas álgebras de Artin $A$ e $B$ quando $B$ for uma extensão cindida de $A$ por um ideal nilpotente, isto é, quando existir um epimorfismo cindido de álgebras $\pi: B \rightarrow A$, cujo núcleo é um ideal nilpotente de $B$ (ver [6,7,9,10]). Quando isso acontece podemos considerar $A$ como uma subálgebra de $B$. Mais ainda, todo módulo sobre $A$ é um módulo sobre $B$ e vice-versa. Para fazer uma conexão entre as categorias dos módulos de tipo finito sobre $A$ e sobre $B$, utilizamos os funtores de mudança de anéis

$$
-\otimes_{A} B, \operatorname{Hom}_{A}\left(B_{A},-\right): \bmod A \rightarrow \bmod B \text { e }-\otimes_{B} A, \operatorname{Hom}_{B}\left(A_{B},-\right): \bmod B \rightarrow \bmod A
$$


(ver [12]). Com eles relacionamos os módulos indecomponíveis em $\bmod A$ e em $\bmod B$, em especial os projetivos e os injetivos. Na verdade, todo $B$-módulo projetivo é isomorfo a um módulo da forma $P \otimes_{A} B$, onde $P_{A}$ é uma $A$-módulo projetivo; e todo $B$-módulo injetivo é isomorfo a um módulo da forma $\operatorname{Hom}_{A}(B, I)$, onde $I$ é um $A$-módulo injetivo.

A partir disso relacionamos resoluções e apresentações projetivas (e injetivas) de um "mesmo" módulo visto como $A$-módulo e como $B$-módulo e, portanto, conseguimos algumas propriedades homológicas da categoria ind $A$ a partir de propriedades ind $B$ (aqui ind $C$ é a subcategoria plena de mod $C$ cujos objetos são representantes das classes de isomorfismo dos $C$-módulos indecomponíveis). Um resultado, envolvendo as dimensões projetivas (dp) e injetivas (di), é o seguinte:

$$
\begin{gathered}
\text { Para todo } A \text {-módulo } M \text { indecomponível, } \\
\text {. se dp } M_{B} \leq 1 \text { então dp } M_{A} \leq 1 \\
\text {. se di } M_{B} \leq 1 \text { então di } M_{A} \leq 1
\end{gathered}
$$

Tal resultado merece destaque pois nos garante duas propriedades de $B$ que são herdadas por $A$ : ser hereditária e ser shod.

Uma pergunta natural é se esse tipo de resultado também é válido para outras classes de álgebras. Estudamos então os conceitos de parte direita $(\mathcal{R})$ e parte esquerda $(\mathcal{L})$ da categoria de módulos [16] que podem ser definidas, para uma álgebra de Artin $C$, por

$$
\begin{aligned}
\mathcal{L} & :=\{X \in \text { ind } C \mid \text { se } Y \text { é predecessor de } X, \text { então dp } Y \leq 1\} \text { e } \\
\mathcal{R} & :=\{X \in \text { ind } C \mid \text { se } X \text { é sucessor de } Y, \text { então di } Y \leq 1\} .
\end{aligned}
$$

Essas subcategorias de ind $C$ nos permitem caracterizar algumas classes de álgebras que são frequentemente estudadas em teoria de representações [5]. Algumas dessas classes estão no seguinte Teorema A de [10]. Sejam $A$ e $B$ álgebras de Artin, tais que $B$ é uma extensão cindida por nilpotente de $A$. Então,

- se $B$ é laura então $A$ também é laura;

- se $B$ colada à esquerda então $A$ também é colada à esquerda;

- se $B$ colada à direita então $A$ também é colada à direita; 
- se $B$ é fracamente shod então $A$ também é fracamente shod;

- se $B$ é shod então $A$ também é shod;

- se $B$ é quase inclinada então $A$ também é quase inclinada.

Mostramos também um resultado análogo para álgebras disfarçadas, porém como estas álgebras são de tipo de representação infinito, precisamos supor que $A$ é de tipo infinito.

As recíprocas desses resultados não são válidas. Para construírmos alguns contra-exemplos restringimos nosso estudo para álgebras de dimensão finita sobre um corpo algebricamente fechado, mas ainda considerando $B$ uma extensão cindida de $A$ por um ideal nilpotente $Q$. Mostramos que a aljava ordinária de $A$ é uma subaljava da aljava ordinária de $B$, um resultado que já era esperado uma vez que $A$ pode ser vista como subálgebra de $B$. Há também uma forte ligação entre as apresentações dessas álgebras, o que nos permitiu caracterizar o ideal $Q$ [6]. Utilizamos tal caracterização para construir exemplos de extensões cindidas por nilpotente. Por fim, para justificar que uma álgebra pertence ou não a uma das classes de álgebras estudadas, fizemos uso da aljava de Auslander-Reiten [8,11] que permite uma "visualização" da categoria dos módulos indecomponíveis e das subcategorias $\mathcal{L}$ e $\mathcal{R}$.

\section{Organização do Trabalho}

No primeiro capítulo fazemos um apanhado de definições e resultados gerais envolvendo categorias e funtores, módulos sobre álgebras, produto tensorial e álgebras de caminhos (ver [1,8,11-13]), que são utilizados no decorrer do trabalho.

Para os demais capítulos foram estudadas as extensões cindidas por nilpotente, principalmente em $[6,7,9,10]$.

No Capítulo 2 definimos extensão cindida por um ideal nilpotente para álgebras de Artin. Estudamos as categorias mod $A$ e mod $B$ através dos funtores de mudanças de anéis, em especial, os módulos projetivos e injetivos. Mostramos ainda que se $A$ é uma álgebra conexa então $B$ também o é. Os resultados foram retirados em sua maioria de $[7,9,10]$.

Já no Capítulo 3 restringimos o estudo para álgebras de dimensão finita sobre um corpo algebricamente fechado e passamos a estudar as caracterizações, feitas em [6], das aljavas ordinárias e apresntações das álgebras $A$ e $B$, bem como do ideal nilpotente $Q$.

O Capítulo 4 é dedicado ao estudo das propriedades homológicas das categorias $\bmod A$ e mod $B$. 
Para isso, incluímos uma seção introdutória contendo definições e algumas propriedades de coberturas projetivas, envolventes injetivas, dimensões projetivas e injetivas, etc. A segunda seção compara as propriedades homológicas dos $A$-módulos e dos $B$-módulos. Ao final mostramos que se $B$ é uma álgebra hereditária (ou shod) então $A$ também é hereditária (ou shod). Tais resultados são em sua maioria de $[7,10]$.

No Capítulo 5 extendemos esse último resultado para outras classes de álgebras. Iniciamos com um estudo das partes direita e esquerda das categorias $\bmod A$ e $\bmod B$. As álgebras laura, colada à direita (à esquerda), fracamente shod, shod, quase inclinada e disfarçada podem ser determinadas utilizando esses conceitos, como em [5]. Provamos que se $B$ pertence a uma dessas classes então $A$ também pertence a mesma classe [10]. Finalmente, na última seção damos alguns exemplos de extensões cindidas, utilizando as técnicas do Capítulo 3 , que mostram que $A$ pode ser de uma dessas classes de álgebras sem que $B$ o seja.

Incluímos um apêndice com algumas características da aljava de Auslander-Reiten que são usadas nos exemplos do Capítulo 5. 


\section{Capítulo 1}

\section{Preliminares}

Começamos nosso trabalho incluindo as definições e os resultados básicos de álgebras artinianas que serão utilizados nos próximos capítulos. Muitos dos resultados terão suas demonstrações omitidas, as quais podem ser encontradas, por exemplo, em [1], [8], [11] e [13]. Na seção de álgebras de caminhos restringimos nosso estudo às álgebras de dimensão finita sobre corpos algebricamente fechados.

Em muitas partes do trabalho utilizamos propriedades de categorias e funtores, por isso incluímos inicialmente uma seção com algumas definições e resultados que serão utilizados. Estes podem ser encontrados por exemplo em [1], [8] e [12].

\subsection{Categorias e Funtores}

Uma categoria $\mathfrak{C}$ é definida por:

- uma classe de objetos de $\mathfrak{C}$, denotada por Ob $\mathfrak{C}$;

- para cada par $(X, Y)$ de objetos de $\mathfrak{C}$ associamos um conjunto chamado de conjunto de morfismos de $X$ para $Y$, denotado por $\operatorname{Hom}_{\mathfrak{C}}(X, Y)$, e tal que se $(X, Y) \neq\left(X^{\prime}, Y^{\prime}\right)$ então $\operatorname{Hom}_{\mathfrak{C}}(X, Y) \cap \operatorname{Hom}_{\mathfrak{C}}\left(X^{\prime}, Y^{\prime}\right)=\emptyset$;

- para cada tripla $(X, Y, Z)$ de objetos de $\mathfrak{C}$, há uma operação de composição de morfismos, denotada por $\circ: \operatorname{Hom}_{\mathfrak{C}}(Y, Z) \times \operatorname{Hom}_{\mathfrak{C}}(X, Y) \rightarrow \operatorname{Hom}_{\mathfrak{C}}(X, Z)$ tal que

$-h \circ(g \circ f)=(h \circ g) \circ f$ para todo $f \in \operatorname{Hom}_{\mathfrak{C}}(X, Y), g \in \operatorname{Hom}_{\mathfrak{C}}(Y, Z), h \in \operatorname{Hom}_{\mathfrak{C}}(Z, W)$;

- para todo objeto $X$ de $\mathfrak{C}$ existe um morfismo $i d_{X}$ em $\operatorname{Hom}_{\mathfrak{C}}(X, X)$, chamado de morfismo 
identidade de $X$, tal que $f \circ i d_{X}=f$ e $i d_{X} \circ g=g$, para todo morfismo $f \in \operatorname{Hom}_{\mathfrak{C}}(X, Y)$ e $g \in \operatorname{Hom}_{\mathfrak{C}}(Z, X)$.

Escreveremos, por abuso de notação, $X \in \mathrm{Ob} \mathfrak{C}$, ou ainda $X \in \mathfrak{C}$ para dizer que $X$ é um objeto da categoria $\mathfrak{C}$ e $f: X \rightarrow Y$ (ou $X \stackrel{f}{\rightarrow} Y$ ) denota que $f \in \operatorname{Hom}_{\mathfrak{C}}(X, Y)$. Além disso, em alguns casos também escreveremos $f g$ no lugar de $f \circ g$.

Dado um morfismo $f: X \rightarrow Y$, uma secção de $f$ é um morfismo $g: Y \rightarrow X$ tal que $f g=i d_{Y}$ e uma retração de $f$ é um morfismo $h: Y \rightarrow X$ tal que $h f=i d_{X}$. Diremos que o morfismo $f: X \rightarrow Y$ é um isomorfismo se existir $h: Y \rightarrow X$ que é uma secção e uma retração de $f$, ou seja, $h f=i d_{X}$ e $f h=i d_{Y}$. Nesse último caso, dizemos que os objetos $X$ e $Y$ são isomorfos $(X \cong Y)$.

Dados os objetos $X_{1}, \ldots, X_{n}$ de $\mathfrak{C}$, a soma direta é um objeto de $\mathfrak{C}$, denotado por $X_{1} \oplus \ldots \oplus X_{n}$, junto com um conjunto de morfismos $u_{i}: X_{i} \rightarrow X_{1} \oplus \ldots \oplus X_{n}$, com $i=1, \ldots, n$, tais que para cada objeto $Z \in \mathrm{Ob} \mathfrak{C}$ e cada conjunto de morfismos $f_{i}: X_{i} \rightarrow Z, i=1, \ldots, n$ em $\mathfrak{C}$, existe um único morfismo $f: X_{1} \oplus \ldots \oplus X_{n} \rightarrow Z$ tal que para cada $i$ vale $f_{i}=f \circ u_{i}$. Também escrevemos $\bigoplus_{i=1}^{n} X_{i}=X_{1} \oplus \ldots \oplus X_{n}$. Cada morfismo $u_{i}$ é chamado de $i$-ésima inclusão.

Uma categoria $\mathfrak{C}$ é dita aditiva se:

- para quaisquer objetos $X_{1}, \ldots, X_{n}$ de $\mathfrak{C}$ existe a soma direta $X_{1} \oplus \ldots \oplus X_{n}$ em $\mathfrak{C}$;

- o conjunto $\operatorname{Hom}_{\mathfrak{C}}(X, Y)$ tem estrutura de grupo abeliano, para cada $X, Y \in \mathfrak{C}$;

- existe um objeto zero, $0 \in \mathrm{Ob} \mathfrak{C}$, tal que o morfismo identidade $i d_{0}$ é o elemento nulo do grupo abeliano $\operatorname{Hom}_{\mathfrak{C}}(0,0)$;

- para $f, g, h$ morfismos em $\mathfrak{C}$, vale $(f+g) \circ h=f \circ h+g \circ h$ e $f \circ(g+h)=f \circ g+f \circ h$ (desde que estas operações estejam definidas).

Para uma categoria aditiva $\mathfrak{C}$, a categoria dual ou oposta, denotada por $\mathfrak{C}^{o p}$ é definida como a categoria cujos objetos são os mesmos de $\mathfrak{C}, \operatorname{Hom}_{\mathfrak{C} o p}(X, Y)=\operatorname{Hom}_{\mathfrak{C}}(Y, X)$ para $X, Y \in \mathrm{Ob} \mathfrak{C}$ e a composição de $f \in \operatorname{Hom}_{\mathfrak{C}^{o p}}(X, Y) \operatorname{com} g \in \operatorname{Hom}_{\mathfrak{C} o p}(Y, Z)$ é $f g \in \operatorname{Hom}_{\mathfrak{C}}(Z, X)=\operatorname{Hom}_{\mathfrak{C}^{o p}}(X, Z)$.

Exemplo 1.1 (Notação matricial) Seja $\bigoplus_{i=1}^{n} X_{i}$ junto com $\left\{u_{i}\right\}_{i}$ a soma direta dos objetos $X_{i}$ 
de uma categoria aditiva $\mathfrak{C}$. Existem morfismos $p_{j}: \bigoplus_{i=1}^{n} X_{i} \rightarrow X_{j}$ (j-ésima projeção) tais que $p_{j} \circ u_{j}=i d_{X_{j}}, p_{j} \circ u_{i}=0$ se $i \neq j$ e $\bigoplus_{i=1}^{n}\left(u_{i} \circ p_{i}\right)=i d_{\oplus X_{i}}$.

Dados os morfismos $f_{i}: X_{i} \rightarrow Y$ e $g_{j}: Y \rightarrow Z_{j}$ em $\mathfrak{C}$, denotamos por $f=\left[\begin{array}{lll}f_{1} & \cdots & f_{n}\end{array}\right]$ : $X_{1} \oplus \ldots \oplus X_{n} \rightarrow Y$ o morfismo tal que $f \circ u_{i}=f_{i}$ para cada $i=1, \ldots, n$ e $g=\left[\begin{array}{c}g_{1} \\ \vdots \\ g_{m}\end{array}\right]: Y \rightarrow Z_{1} \oplus \ldots \oplus Z_{m}$ o morfismo tal que $p_{i} \circ g=g_{i}$ para cada $i=1, \ldots, m$.

Se $X=X_{1} \oplus \ldots \oplus X_{n}$ e $Z=Z_{1} \oplus \ldots \oplus Z_{m}$ então um morfismo $h: X \rightarrow Z$ em $\mathfrak{C}$ é denotado pela matriz $h:\left[h_{i j}\right]=\left[\begin{array}{cccc}h_{11} & h_{12} & \cdots & h_{1 n} \\ h_{21} & h_{22} & \cdots & h_{2 n} \\ \vdots & \vdots & \ddots & \vdots \\ h_{m 1} & h_{m 2} & \cdots & h_{m n}\end{array}\right]$ onde $h_{i j}=p_{i} \circ h \circ u_{j}: X_{j} \rightarrow Z_{i}$.

Dizemos que uma categoria $\mathfrak{D}$ é uma subcategoria de $\mathfrak{C}$ se:

- a classe $\mathrm{Ob} \mathfrak{D}$ é uma subclasse de $\mathrm{Ob} \mathfrak{C}$;

- se $X, Y \in \mathrm{Ob} \mathfrak{D}$, então $\operatorname{Hom}_{\mathfrak{D}}(X, Y) \subseteq \operatorname{Hom}_{\mathfrak{C}}(X, Y)$;

- a composição de $\mathfrak{D}$ é a mesma de $\mathfrak{C} ;$

- para cada objeto $X$ de $\mathfrak{D}$, o morfismo identidade em $\operatorname{Hom}_{\mathfrak{D}}(X, X)$ coincide com o morfismo identidade em $\operatorname{Hom}_{\mathfrak{C}}(X, X)$.

Uma subcategoria $\mathfrak{D}$ de $\mathfrak{C}$ é dita plena se $\operatorname{Hom}_{\mathfrak{D}}(X, Y)=\operatorname{Hom}_{\mathfrak{C}}(X, Y)$ para todos os objetos $X$ e $Y$ em $\mathfrak{D}$.

\section{Funtores}

Sejam $\mathfrak{C}$ e $\mathfrak{D}$ categorias, define-se um funtor covariante $F: \mathfrak{C} \rightarrow \mathfrak{D}$ associando para cada objeto $X$ de $\mathfrak{C}$ um objeto $F X$ (ou $F(X))$ de $\mathfrak{D}$ e para cada morfismo $f: X \rightarrow Y$ em $\mathfrak{C}$, um morfismo $F f: F X \rightarrow F Y($ ou $F(f))$ em $\mathfrak{D}$ tal que: 
- $F(g f)=(F g)(F f)$, para todos $f$ e $g$ morfismos em $\mathfrak{C}$;

- $F i d_{X}=i d_{F X}$, para todo objeto $X$ de $\mathfrak{C}$.

Define-se um funtor contravariante $F: \mathfrak{C} \rightarrow \mathfrak{D}$ associando para cada objeto $X$ de $\mathfrak{C}$ um objeto $F X$ de $\mathfrak{D}$ e para cada morfismo $f: X \rightarrow Y$ em $\mathfrak{C}$, um morfismo $F f: F Y \rightarrow F X$ em $\mathfrak{D}$ tal que:

- $F(g f)=(F f)(F g)$, para todos $f$ e $g$ morfismos em $\mathfrak{C}$;

- $F i d_{X}=i d_{F X}$, para todo objeto $X$ de $\mathfrak{C}$.

Sejam $\mathfrak{C}$ e $\mathfrak{D}$ categorias aditivas. Um funtor $F: \mathfrak{C} \rightarrow \mathfrak{D}$ é dito aditivo se dados $X$ e $Y$ em $\mathfrak{C}$, temos $F(X \oplus Y) \cong F(X) \oplus F(Y)$ em $\mathfrak{D}$ e se $f, g \in \operatorname{Hom}_{\mathfrak{C}}(X, Y)$ então $F(f+g)=F(f)+F(g)$.

Sejam $F, G$ funtores covariantes da categoria $\mathfrak{C}$ na categoria $\mathfrak{D}$. Um morfismo funtorial ou transformação natural $\Phi: F \rightarrow G$ é uma família $\left\{\Phi_{X}\right\}_{X \in O b \mathfrak{C}}$ de morfismos $\Phi_{X}: F X \rightarrow G X$ de $\mathfrak{D}$ tal que, se $f: X \rightarrow Y$ é um morfismo de $\mathfrak{C}$, então $\Phi_{Y} \circ F f=G f \circ \Phi_{X}$, ou seja, o seguinte diagrama é comutativo:

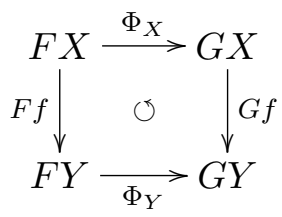

Sejam $F, G$ são funtores contravariantes da categoria $\mathfrak{C}$ na categoria $\mathfrak{D}$. Um morfismo funtorial ou transformação natural $\Phi: F \rightarrow G$ é uma família $\left\{\Phi_{X}\right\}_{X \in O b \mathfrak{C}}$ de morfismos $\Phi_{X}: F X \rightarrow G X$ de $\mathfrak{D}$ tal que, se $f: X \rightarrow Y$ é um morfismo de $\mathfrak{C}$, então $\Phi_{X} \circ F f=G f \circ \Phi_{Y}$, ou seja, o seguinte diagrama é comutativo:

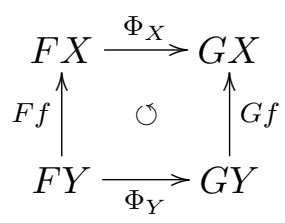

A composição de morfismos funtoriais definida por $(\Phi \Psi)_{X}=\Phi_{X} \Psi_{X}$ é ainda um morfismo funtorial. Para cada funtor $F$ tem-se um morfismo funtorial identidade $i d_{F}: F \rightarrow F$ dado por $\left(i d_{F}\right)_{X}=i d_{F X}$. Um morfismo funtorial $\Phi: F \rightarrow G$ é dito isomorfismo funtorial se cada $\Phi_{X}$ for um isomorfismo em $\mathfrak{D}$. Nesse caso existe um morfismo funtorial $\Psi: G \rightarrow F$ tal que $\Phi \Psi=i d_{G}$ e 
$\Psi \Phi=i d_{F}$. Utilizaremos $F \approx G$ para dizer que existe um isomorfismo funtorial entre $F$ e $G$. A composição de isomorfismos funtoriais é também um isomorfismo funtorial.

Sejam $\mathfrak{C}$ e $\mathfrak{D}$ categorias, $F: \mathfrak{C} \rightarrow \mathfrak{D}$ e $G: \mathfrak{D} \rightarrow \mathfrak{C}$ funtores. Dizemos que $F$ é adjunto à esquerda de $G$ ou $G$ é adjunto à direita de $F$ ou ainda que o par $(F, G)$ é adjunto se para todos os objetos $X \in \mathfrak{C}$ e $M \in \mathfrak{D}$ existe uma bijeção $\Phi_{(X, M)}: \operatorname{Hom}_{\mathfrak{C}}(X, G M) \longrightarrow \operatorname{Hom}_{\mathfrak{D}}(F X, M)$ que é funtorial em cada variável.

Um funtor (covariante) $F: \mathfrak{C} \rightarrow \mathfrak{D}$ é dito uma equivalência entre as categorias $\mathfrak{C}$ e $\mathfrak{D}$ se existir um funtor $G: \mathfrak{D} \rightarrow \mathfrak{C}$ tal que $F G \approx i d_{\mathfrak{D}}$ e $G F \approx i d_{\mathfrak{C}}$. Nesse caso, dizemos que $G$ é um quase-inverso de $F$ e que as categorias $\mathfrak{C}$ e $\mathfrak{D}$ são equivalentes $(\mathfrak{C} \approx \mathfrak{D})$. Um funtor contravariante $F: \mathfrak{C} \rightarrow \mathfrak{D}$ é dito uma equivalência entre as categorias $\mathfrak{C}$ e $\mathfrak{D}$ se o funtor covariante induzido $F$ uma equivalência de categorias. Nesse caso, $F$ é chamado de dualidade.

Um funtor covariante $F: \mathfrak{C} \rightarrow \mathfrak{D}$ induz, para cada par $(X, Y)$ de objetos de $\mathfrak{C}$, uma aplicação $F: \operatorname{Hom}_{\mathfrak{C}}(X, Y) \rightarrow \operatorname{Hom}_{\mathfrak{D}}(F X, F Y)$ dada por $f \mapsto F f$. Se esta aplicação é injetora, dizemos que o funtor $F$ é fiel. Se for sobrejetora, dizemos que o funtor $F$ é pleno. O funtor $F$ é dito denso se para cada objeto $M$ de $\mathfrak{D}$ existir um objeto $X$ de $\mathfrak{C}$ tal que $M$ e $F X$ são isomorfos em $\mathfrak{D}$.

Proposição 1.1 Sejam $\mathfrak{C}$ e $\mathfrak{D}$ categorias. Um funtor $F: \mathfrak{C} \rightarrow \mathfrak{D}$ é uma equivalência de categorias se, e somente se, $F$ é fiel, pleno e denso.

\section{2 Álgebras e Módulos}

Seja $R$ um anel comutativo com unidade. Uma $R$-álgebra $A$ é um anel com unidade que ao mesmo tempo é um módulo sobre $R$ e tal que para todo $x \in R$ e para todo $a, b \in A$ vale:

$$
(a b) x=a(b x)=(a x) b .
$$

A toda $R$-álgebra $A$ corresponde uma outra $R$-álgebra, chamada de álgebra oposta $A^{\text {op }}$ que tem a mesma estrutura de $R$-módulo de $A$, mas a multiplicação $*$ é definida por $a * a^{\prime}:=a^{\prime} a$ para $\operatorname{todos} a, a^{\prime} \in A$.

Uma $R$-álgebra $A$ é dita uma álgebra de Artin se $R$ é um anel artiniano e $A$ é um $R$-módulo de tipo finito (isto é, finitamente gerado).

Daqui para frente, caso não se faça menção ao contrário, as álgebras serão consideradas $R$-álgebras 
de Artin.

Exemplo 1.2 (Álgebra de Matrizes) Seja $k$ um corpo. O conjunto $M_{n}(k)$, das matrizes $n \times n$ com coeficientes em $k$, munido das operações usuais de matrizes, é uma k-álgebra de Artin de dimensão $n^{2}$.

Um $R$-submódulo $B$ da $R$-álgebra $A$ é uma $R$-subálgebra de $A$ se $1_{A} \in B$ e $b b^{\prime} \in B, \forall b, b^{\prime} \in B$.

Um $R$-submódulo $\mathcal{I}$ de uma $R$-álgebra $A$ é um ideal à direita de $A$ se $\alpha a \in \mathcal{I}$, para todo $\alpha \in \mathcal{I}$ e para todo $a \in A$. Se $a \alpha \in \mathcal{I}, \forall \alpha \in \mathcal{I}, \forall a \in A$ então $\mathcal{I}$ é um ideal à esquerda de $A$. Se $\mathcal{I}$ é um ideal à direita e um ideal à esquerda, então $\mathcal{I}$ é um ideal bilateral de $A$ ou simplesmente um ideal de $A$.

Exemplo 1.3 Consideremos $\mathcal{I}$ um ideal de uma $R$-álgebra A. Seja $\frac{A}{\mathcal{I}}$ o conjunto das classes módulo $\mathcal{I}$ da forma $a+\mathcal{I}=\{a+\alpha: \quad \alpha \in \mathcal{I}\}$, para todo $a \in A$. Então, $\frac{A}{\mathcal{I}}$ tem uma estrutura de $R$ módulo dada por $(a+\mathcal{I})+(b+\mathcal{I}):=(a+b)+\mathcal{I}$ e $(a+\mathcal{I}) x:=$ ax $+\mathcal{I}$, para todo a, $b \in$ A e para todo $x \in R$ e tem uma estrutura de anel dada por $(a+\mathcal{I})(b+\mathcal{I}):=a b+\mathcal{I}$, para todo a, $b \in A$. Além disso, essas duas estruturas são compativeis, ou seja, para todos $a, b \in A$ e para todo $x \in R$ : $((a+\mathcal{I})(b+\mathcal{I})) x=(a+\mathcal{I})((b+\mathcal{I}) x)=((a+\mathcal{I}) x)(b+\mathcal{I})$. Portanto, $\frac{A}{\mathcal{I}}$ é uma R-álgebra, a qual chamamos de álgebra quociente de $A$ por $\mathcal{I}$.

Seja $\mathcal{I}$ um ideal à direita da $R$-álgebra $A$. Um subconjunto $S \subseteq \mathcal{I}$ é dito um gerador do ideal $\mathcal{I}$ se cada elemento $\omega$ de $\mathcal{I}$ possa ser escrito como $\omega=\sum_{i=1}^{n} \omega_{i} a_{i}$ onde $\omega_{i}=\prod_{j=1}^{t} s_{j}$ e cada $s_{j} \in S$. Se $S$ for finito, $\mathcal{I}$ é dito finitamente gerado.

Diremos que um ideal $\mathcal{I}$ de uma $R$-álgebra $A$ é nilpotente se existir um inteiro positivo $n$ tal que $\mathcal{I}^{n}=0$, onde $\mathcal{I}^{n}:=\left\{\sum_{i=1}^{n} \alpha_{1_{i}} \alpha_{2_{i}} \ldots \alpha_{n_{i}} \mid \alpha_{j_{i}} \in \mathcal{I}\right\}$.

Exemplo 1.4 Seja $k$ um corpo. O conjunto $A=\left(\begin{array}{cc}k & 0 \\ k & k\end{array}\right):=\left\{\left(\begin{array}{ll}x & 0 \\ y & z\end{array}\right) \mid x, y, z \in k\right\}$ é uma k-subálgebra da álgebra de matrizes $M_{2}(k)$.

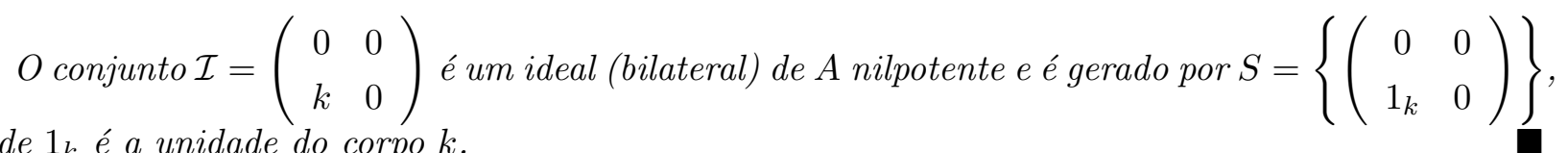

onde $1_{k}$ é a unidade do corpo $k$. 
Um ideal $\mathcal{I}$ de uma $R$-álgebra $A$ é dito maximal se não existir um ideal $\overline{\mathcal{I}} \neq \mathcal{I}, \overline{\mathcal{I}} \neq A$ tal que $\mathcal{I} \subset \overline{\mathcal{I}} \subset A$.

Proposição 1.2 Sejam A uma R-álgebra de Artin e $\mathcal{I}$ um ideal de A. São equivalentes:

1. I é o maior ideal nilpotente.

2. I é a interseção de todos os ideais maximais.

Um ideal com as propriedades da proposição acima é chamado de radical (de Jacobson) de $A$ e é denotado por rad $A$.

\section{Morfismos de álgebras}

Sejam $A$ e $B$ duas $R$-álgebras. Um morfismo ou homomorfismo de $R$-álgebras de $A$ em $B$ é uma aplicação $\phi: A \rightarrow B$ que é $R$-linear e é um homomorfismo de anéis, isto é, para todos $a_{1}, a_{2} \in A$ e $x, y \in R$ :

- $\phi\left(a_{1} x+a_{2} y\right)=\phi\left(a_{1}\right) x+\phi\left(a_{2}\right) y$;

- $\phi\left(a_{1} a_{2}\right)=\phi\left(a_{1}\right) \phi\left(a_{2}\right)$;

- $\phi\left(1_{A}\right)=1_{B}$

Se $\phi$ é injetora então $\phi$ é dita um monomorfismo de álgebras. Se $\phi$ é sobrejetora então $\phi$ é dita um epimorfismo de álgebras. E, se $\phi$ é bijetora, então $\phi$ é dita um isomorfismo de álgebras. Neste último caso, dizemos que as álgebras $A$ e $B$ são isomorfas e denotamos por $A \cong B$.

Exemplo 1.5 Se $A$ é uma $R$-subálgebra de $B$, então a aplicação $\iota: A \rightarrow B$, definida por $\iota(a)=a$, é um monomorfismo de álgebras, chamado de inclusão canônica.

Se $\mathcal{I}$ é um ideal bilateral de $A$ então, a aplicação $\pi: A \rightarrow \frac{A}{\mathcal{I}}$, definida por $\pi(a)=a+\mathcal{I}$ é um epimorfismo de álgebras e é chamado de projeção canônica.

Um monomorfismo é dito monomorfismo cindido quando possuir uma retração e um epimorfismo é dito epimorfismo cindido quando possuir uma secção.

Seja $\phi: A \rightarrow B$ um morfismo de álgebras. Então: 
1. A imagem de $\phi(\operatorname{Im} \phi:=\{\phi(a) \mid a \in A\})$ é uma subálgebra de $B$;

2. $\phi(0)=0$;

3. $\phi(-a)=-\phi(a)$;

4. O núcleo de $\phi$ ( Nuc $\phi:=\{a \in A \mid \phi(a)=0\}$ ) é um ideal bilateral de $A$;

5. $\phi$ é injetora se, e somente se, Nuc $\phi=0$;

6. $\phi(\operatorname{rad} A) \subseteq \operatorname{rad} B$.

Proposição 1.3 (Teorema do isomorfismo para álgebras) Se $\phi: A \rightarrow B$ é um epimorfismo de álgebras, então

$$
\frac{A}{\operatorname{Nuc} \phi} \cong B
$$

\section{Módulos}

Nessa seção as álgebras serão $R$-álgebras de Artin, onde $R$ é uma anel comutativo com unidade.

Seja $A$ uma $R$-álgebra. Um $A$-módulo à direita $M$ é simplesmente um módulo à direita sobre o anel $A$. Neste caso, $M$ é dotado de uma estrutura natural de $R$-módulo à direita, dada por $m x:=m\left(1_{A} x\right)$, para todo $m \in M$ e para todo $x \in R$. Como $R$ é comutativo, $M$ também tem estrutura de $R$-módulo à esquerda. Além disso, se $x \in R, m \in M$ e $a \in A$ vale

$$
(x m) a=x(m a)=m(x a) .
$$

Analogamente, define-se $A$-módulos à esquerda.

Usaremos a seguinte notação: $M_{A}$ denota um $A$-módulo à direita e ${ }_{A} M$ denota um $A$-módulo à esquerda. Em muitos casos, omitiremos enunciados envolvendo módulos à esquerda, pois estes são inteiramente análogos ao caso dos módulos à direita.

Exemplo 1.6 Sejam $M_{A}$ um A-módulo e $\mathcal{I}$ um ideal de A, então o conjunto

$$
M \mathcal{I}:=\left\{\sum_{j} m_{j} \alpha_{j} \mid m_{j} \in M, \alpha_{j} \in \mathcal{I}\right\}
$$


é um A-submódulo de $M_{A}$.

Se $M$ for anulado pelo ideal $\mathcal{I}$, isto é, se $M \mathcal{I}=0$, então $M$ possui uma estrutura natural de $\frac{A}{\mathcal{I}}$-módulo, dada por $m \cdot(a+\mathcal{I}):=$ ma, para $m \in M$ e $a \in A$.

Se $M$ é um $A$-módulo à direita então o radical de $M$, denotado por rad $M_{A}$, é o $A$-submódulo $M(\operatorname{rad} A) . \mathrm{O}$ radical do $A$-módulo $A_{A}$ é $\operatorname{rad} A_{A}=A \operatorname{rad} A=\operatorname{rad} A$. Vale ainda que $\operatorname{rad}(M \oplus N)_{A}=$ $\operatorname{rad} M_{A} \oplus \operatorname{rad} N_{A}$; e se $N_{A}$ é um submódulo de $M_{A} \operatorname{com} N \subseteq \operatorname{rad} M$, então $\operatorname{rad} \frac{M}{N}=\frac{\operatorname{rad} M}{N}$.

Proposição 1.4 (Lema de Nakayama) Sejam $M_{A}$ um A-módulo de tipo finito e $N_{A}$ um submódulo de $M_{A}$. Então $N \subseteq \operatorname{rad} M$ se, e somente se, $N+L=M$ implica que $L=M$, para todo submódulo $L_{A} \subseteq M_{A}$.

Sejam $A$ e $B$ duas $R$-álgebras. Um conjunto $M$ que tem estrutura de $A$-módulo à esquerda e estrutura de $B$-módulo à direita é um $(A-B)$-bimódulo se estas estruturas forem compatíveis, isto é, se $a(m b)=(a m) b$ para todo $a \in A, m \in M$ e $b \in B$.

Denotaremos um $(A-B)$-bimódulo $M$ por ${ }_{A} M_{B}$.

\section{Morfismos de módulos}

Sejam $M$ e $N$ dois $A$-módulos à direita. Um morfismo de $A$-módulos ou homomorfismo de $A$-módulos é uma aplicação $f: M \rightarrow N$ tal que $f\left(m_{1} a+m_{2} b\right)=f\left(m_{1}\right) a+f\left(m_{2}\right) b$ para todos $m_{1}, m_{2} \in M$ e $a, b \in A$. Um morfismo de $A$-módulos é, claramente, $R$-linear.

Se $M$ e $N$ são $(A$ - $B$ )-bimódulos, uma aplicação que é um morfismo de $A$-módulos à direita e de $B$-módulos à esquerda é dita um morfismo de $(A-B)$-bimódulos.

Se $f: M \rightarrow N$ é um morfismo de $A$-módulos, dizemos que $f$ é um monomorfismo se $f$ é injetora e que $f$ é um epimorfismo se $f$ é sobrejetora. Se $f$ é bijetora, então dizemos que é um isomorfismo de módulos, neste caso dizemos que $M$ e $N$ são isomorfos e denotamos por $M \cong N$.

Para um morfismo de módulos à direita (ou à esquerda) $f: M \rightarrow N$ valem propriedades análogas as enunciadas para um morfismo de álgebras:

1. A imagem de $f$ e o núcleo de $f$ são submódulos de $N$ e $M$ respectivamente;

2. $f(0)=0$; 
3. $f(-m)=-f(m)$;

4. $f$ é um monomorfismo se, e somente se, Nuc $f=0$;

5. $f$ é um epimorfismo se, e somente se, Conuc $f:=\frac{M}{\operatorname{Im} f}=0$;

6. $f\left(\operatorname{rad} M_{A}\right) \subseteq \operatorname{rad} N_{A}\left(\operatorname{ou} f\left(\operatorname{rad}_{A} M\right) \subseteq \operatorname{rad}_{A} N\right)$.

Denotamos por $\operatorname{Hom}_{A}(M, N)$ o conjunto dos morfismos (de $A$-módulos) de $M$ em $N$. Tal conjunto tem estrutura de $R$-módulo com as seguintes operações: se $f, g \in \operatorname{Hom}_{A}(M, N)$ e $x \in R$ definimos $(f+g)(m):=f(m)+g(m)$ e $(f x)(m):=x f(m)$, para todo $m \in M$.

Denotaremos por Mod $A$ ( $A$-Mod) a categoria aditiva cujos objetos são os $A$-módulos à direita (à esquerda) e os morfismos são os morfismos de $A$-módulos. Denotaremos também por mod $A(A$-mod $)$ a subcategoria plena de Mod $A$ ( $A$-Mod ) cujos objetos são os $A$-módulos de tipo finito.

Seja $M_{A}$ um $A$-módulo. Definimos um funtor covariante $\operatorname{Hom}_{A}(M,-): \operatorname{Mod} A \rightarrow \operatorname{Mod} R$ que a cada $A$-módulo $N_{A}$ associa o $R$-módulo $\operatorname{Hom}_{A}(M, N)$ e a cada morfismo de $A$-módulos $f: L_{A} \rightarrow N_{A}$ associa o morfismo $R$-linear $\operatorname{Hom}_{A}(M, f): \operatorname{Hom}_{A}(M, L) \rightarrow \operatorname{Hom}_{A}(M, N)$, onde $\operatorname{Hom}_{A}(M, f)(g):=$ $f g$. Analogamente, definimos o funtor contravariante $\operatorname{Hom}_{A}(-, M): \operatorname{Mod} A \rightarrow \operatorname{Mod} R$ que a cada módulo $N_{A}$ associa o $R$-módulo $\operatorname{Hom}_{A}(M, N)$ e a cada morfismo de $A$-módulos $f: L_{A} \rightarrow N_{A}$ associa o morfismo $R$-linear $\operatorname{Hom}_{A}(f, M): \operatorname{Hom}_{A}(N, M) \rightarrow \operatorname{Hom}_{A}(L, M)$, onde $\operatorname{Hom}_{A}(f, M)(g):=g f$.

Dadas as $R$-álgebras $A$ e $B$ vale:

1. para ${ }_{A} M_{B}$ e $N_{B}, \operatorname{Hom}_{B}(M, N)$ é um $A$-módulo à direita com $(f a)(m):=f(a m)$;

2. para ${ }_{A} M_{B}$ e ${ }_{A} N, \operatorname{Hom}_{A}(M, N)$ é um $B$-módulo à esquerda com $(b f)(m):=f(m b)$;

3. para ${ }_{A} M$ e ${ }_{A} N_{B}, \operatorname{Hom}_{A}(M, N)$ é um $B$-módulo à direita com $(f b)(m):=f(m) b$;

4. para $M_{B}$ e ${ }_{A} N_{B}, \operatorname{Hom}_{B}(M, N)$ é um $A$-módulo à esquerda com $(a f)(m):=a f(m)$.

Podemos então definir outros funtores, como por exemplo: $\operatorname{Hom}_{A}\left(-,{ }_{A} N_{B}\right): \operatorname{Mod} A^{o p} \rightarrow \operatorname{Mod} B$ e $\operatorname{Hom}_{B}\left(-,{ }_{A} N_{B}\right): \operatorname{Mod} B \rightarrow \operatorname{Mod} A^{o p}$. Todos esses funtores são aditivos.

Exemplo 1.7 Dado um A-módulo $M_{A} \in \operatorname{Mod} A$ temos que $\operatorname{Hom}_{A}\left({ }_{A} A_{A}, M_{A}\right) \cong M_{A}$. Mais ainda, esse isomorfismo é funtorial, ou seja, $\operatorname{Hom}_{A}\left({ }_{A} A_{A},-\right) \approx i d_{\operatorname{Mod} A}$. 
Se $M_{A}$ e $N_{A}$ são de tipo finito, então $\operatorname{Hom}_{A}\left(M_{A}, N_{A}\right)$ é também de tipo finito. Podemos então considerar os funtores "restrições" como $\operatorname{Hom}_{A}\left(-,{ }_{A} N_{B}\right): \bmod A^{o p} \rightarrow \bmod B$ por exemplo.

Exemplo 1.8 (Funtor dual) $O$ funtor contravariante $D:=\operatorname{Hom}_{R}(-, R): \bmod A \rightarrow \bmod A^{\text {op }}$ é uma dualidade cujo funtor quase-inverso é $D:=\operatorname{Hom}_{R}(-, R): \bmod A^{\text {op }} \rightarrow \bmod A$, ou seja, $D^{2}:=D \circ D \approx i d_{\bmod A}$ e portanto $\bmod A \approx \bmod A^{o p}$.

Para cada A-módulo $M$, o $A^{o p}$-módulo DM é chamado de dual de $M$. Dados os A-módulos $M$ e $N$, vale ainda que $\operatorname{Hom}_{A}(M, N) \cong \operatorname{Hom}_{A^{o p}}(D N, D M)$.

\section{Sequência exata de módulos}

Uma sequência de $A$-módulos e de morfismos de $A$-módulos

$$
\cdots \longrightarrow M_{i+1} \stackrel{f_{i+1}}{\longrightarrow} M_{i} \stackrel{f_{i}}{\longrightarrow} M_{i-1} \stackrel{f_{i-1}}{\longrightarrow} \cdots
$$

é dita exata em $M_{i}$ se $\operatorname{Im} f_{i+1}=$ Nuc $f_{i}$. Tal sequência é dita exata se for exata em todo $M_{i}$.

Observemos que dado um morfismo de $A$-módulos $f: M \rightarrow N$, temos que $f$ é um monomorfismo se, e somente se, a sequência $0 \rightarrow M \stackrel{f}{\rightarrow} N$ for exata; e $f$ é um epimorfismo se, e somente se, a sequência $M \stackrel{f}{\rightarrow} N \rightarrow 0$ for exata.

Uma sequência exata de $A$-módulos da forma $0 \longrightarrow L \stackrel{f}{\longrightarrow} M \stackrel{g}{\longrightarrow} N \longrightarrow 0$ é chamada de sequência exata curta. Dizemos que uma sequência exata curta cinde se $f$ tiver uma secção, ou equivalentemente, se $g$ tiver uma retração. Nesse caso, $M \cong L \oplus N$.

O funtor dual é um funtor exato, ou seja, se $0 \rightarrow L \rightarrow M \rightarrow N \rightarrow 0$ é uma sequência exata curta, então a sequência induzida $0 \rightarrow D N \rightarrow D M \rightarrow D L \rightarrow 0$ também é exata.

Proposição 1.5 Seja A uma R-álgebra.

a) A sequência $0 \rightarrow L_{A} \stackrel{f}{\rightarrow} M_{A} \stackrel{g}{\rightarrow} N_{A}$ é exata se, e somente se, pra todo $X_{A}$, a sequência de $R$-módulos $0 \longrightarrow \operatorname{Hom}_{A}(X, L) \stackrel{\operatorname{Hom}_{A}(X, f)}{\longrightarrow} \operatorname{Hom}_{A}(X, M) \stackrel{\operatorname{Hom}_{A}(X, g)}{\longrightarrow} \operatorname{Hom}_{A}(X, N)$ é exata.

b) A sequência $L_{A} \stackrel{f}{\rightarrow} M_{A} \stackrel{g}{\rightarrow} N_{A} \rightarrow 0$ é exata se, e somente se, pra todo $X_{A}$, a sequência de $R$-módulos $0 \longrightarrow \operatorname{Hom}_{A}(N, X) \stackrel{\operatorname{Hom}_{A}(g, X)}{\longrightarrow} \operatorname{Hom}_{A}(M, X) \stackrel{\operatorname{Hom}_{A}(f, X)}{\longrightarrow} \operatorname{Hom}_{A}(L, X)$ é exata. 
c) A sequência $0 \rightarrow L_{A} \stackrel{f}{\rightarrow} M_{A} \stackrel{g}{\rightarrow} N_{A} \rightarrow 0$ é exata e cinde se, e somente se, para todo $X_{A}, a$ sequência de $R$-módulos $0 \longrightarrow \operatorname{Hom}_{A}(X, L) \longrightarrow \operatorname{Hom}_{A}(X, M) \longrightarrow \operatorname{Hom}_{A}(X, N) \longrightarrow 0$ é exata.

d) A sequência $0 \rightarrow L_{A} \stackrel{f}{\rightarrow} M_{A} \stackrel{g}{\rightarrow} N_{A} \rightarrow 0$ é exata e cinde se, e somente se, para todo $X_{A}, a$ sequência de R-módulos $0 \longrightarrow \operatorname{Hom}_{A}(N, X) \longrightarrow \operatorname{Hom}_{A}(M, X) \longrightarrow \operatorname{Hom}_{A}(L, X) \longrightarrow 0$ é exata.

\section{Módulos indecomponíveis, simples, projetivos e injetivos}

Seja $A$ uma $R$-álgebra de Artin. Lembraremos nessa seção propriedades de algumas classes de módulos que são importantes no estudo da categoria de módulos de tipo finito. Consideraremos somente $A$-módulos à direita e de tipo finito.

Um $A$-módulo $M_{A}$ não nulo é dito indecomponível se $M=M_{1} \oplus M_{2}$ implicar que $M_{1}=0$ ou $M_{2}=0$.

Indicaremos por ind $A$ a subcategoria plena de $\bmod A$ que consiste de um conjunto completo de representantes das classes de isomorfismos dos $A$-módulos indecomponíveis de tipo finito. Muitas vezes, por abuso de notação, escreveremos $M \in$ ind $A$ para indicar que $M$ é um $A$-módulo indecomponível.

Proposição 1.6 (Teorema de Krull-Schmidt) Seja A uma R-álgebra de Artin, então todo Amódulo de tipo finito se decompõe como soma direta finita de A-módulos indecomponíveis de tipo finito. Mais ainda, tal decomposição é única a menos de isomorfismo e ordem dos "fatores".

Um $A$-módulo $S_{A}$ não nulo é dito simples se os seus únicos submódulos são os triviais, ou seja, $S_{A}$ e 0.

Definição 1.1 Um A-módulo $P_{A}$ é dito projetivo se possuir as seguintes propriedades equivalentes:

1. para todo epimorfismo $f: M_{A} \rightarrow N_{A}$ e todo morfismo $g: P_{A} \rightarrow N_{A}$ existe um morfismo 
$\bar{g}: P_{A} \rightarrow M_{A}$ tal que $f \bar{g}=g$, ou seja, o seguinte diagrama comuta:

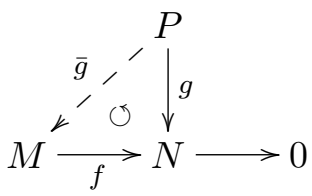

2. $P_{A}$ é um somando direto do A-módulo $A^{(\Lambda)}=\bigoplus_{\lambda \in \Lambda} A_{\lambda}$ onde $A_{\lambda}=A$ para todo $\lambda \in \Lambda$.

3. toda sequência exata curta da forma $0 \longrightarrow M \stackrel{f}{\longrightarrow} N \stackrel{g}{\longrightarrow} P \longrightarrow 0$ cinde.

4. o funtor $\operatorname{Hom}_{A}(P,-): \operatorname{Mod} A \rightarrow \operatorname{Mod} R$ é exato, ou seja, dada uma sequência exata curta $0 \longrightarrow L \stackrel{f}{\longrightarrow} M \stackrel{g}{\longrightarrow} N \longrightarrow 0$ em Mod A, então a sequência induzida em Mod $R$, $0 \longrightarrow \operatorname{Hom}_{A}(P, L) \longrightarrow \operatorname{Hom}_{A}(P, M) \longrightarrow \operatorname{Hom}_{A}(P, N) \longrightarrow 0$ também é exata.

Os indecomponíveis que aparecem na decomposição do $A$-módulo $A_{A}$ formam uma lista completa dos $A$-módulos projetivos indecomponíveis. Os outros projetivos são somas destes.

Definição 1.2 Um A-módulo $I_{A}$ é dito injetivo se possuir as seguintes propriedades equivalentes:

1. para todo monomorfismo $f: M_{A} \rightarrow N_{A}$ e todo morfismo $g: M_{A} \rightarrow I_{A}$ existe um morfismo $\bar{g}: N_{A} \rightarrow I_{A}$ tal que $\bar{g} f=g$, ou seja, o seguinte diagrama comuta:

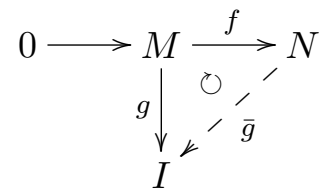

2. toda sequência exata curta da forma $0 \longrightarrow I \stackrel{f}{\longrightarrow} M \stackrel{g}{\longrightarrow} N \longrightarrow 0$ cinde.

3. o funtor $\operatorname{Hom}_{A}(-, I): \operatorname{Mod} A \rightarrow \operatorname{Mod} R$ é exato, ou seja, dada uma sequência exata curta $0 \longrightarrow L \stackrel{f}{\longrightarrow} M \stackrel{g}{\longrightarrow} N \longrightarrow 0$ em Mod $A$, então a sequência induzida em Mod $R$, $0 \longrightarrow \operatorname{Hom}_{A}(N, I) \longrightarrow \operatorname{Hom}_{A}(M, I) \longrightarrow \operatorname{Hom}_{A}(L, I) \longrightarrow 0$ também é exata 
Se $P_{A}$ é um $A$-módulo projetivo então o dual $D P$ é um $A^{o p}$-módulo injetivo e reciprocamente, se $I_{A}$ é um $A$-módulo injetivo então $D I$ é um $A^{o p}$-módulo projetivo.

\subsection{Produto tensorial de módulos}

Para essa seção continuaremos considerando $R$ um anel comutativo com unidade e $R$-álgebras de Artin.

Sejam $M_{A}$ e ${ }_{A} N A$-módulos e $W$ um $R$-módulo. Uma função $g: M \times N \rightarrow W$ é dita $A$-bilinear se para todos $m, m^{\prime} \in M, n, n^{\prime} \in N, x, x^{\prime} \in R$ e $a \in A$,

i) $g\left(m x+m^{\prime} x^{\prime}, n\right)=g(m, n) x+g\left(m^{\prime}, n\right) x^{\prime}$

ii) $g\left(m, n x+n^{\prime} x^{\prime}\right)=g(m, n) x+g\left(m, n^{\prime}\right) x^{\prime}$

iii) $g(m a, n)=g(m, a n)$.

Um produto tensorial de $M$ e $N$ é um par $(T, \tau)$, onde $T$ é um $R$-módulo e $\tau: M \times N \rightarrow T$ é uma aplicação $A$-bilinear, que tem a seguinte propriedade: para cada par $(W, g)$, onde $W$ é um $R$-módulo e $g: M \times N \rightarrow W$ é $A$-bilinear, existe uma única aplicação $R$-linear $\bar{g}: T \rightarrow W$ tal que $\bar{g} \tau=g$, ou seja, que o seguinte diagrama é comutativo:

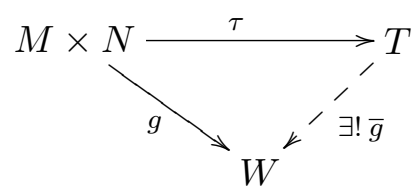

Dados os $A$-módulos $M_{A}$ e ${ }_{A} N$ existe, e é unico (a menos de isomorfismo), o produto tensorial de $M$ e $N$. Denotamos $T$ por $M \otimes_{A} N, \tau$ é chamada de função tensorial e $\tau(m, n)=m \otimes n$ são ditos tensores. Temos que $\tau(M \times N)$ gera $M \otimes_{A} N$, e então podemos escrever para cada $t \in M \otimes_{A} N$, $t=\sum_{i=1}^{n}\left(m_{i} \otimes n_{i}\right)$. Portanto, se $M$ e $N$ são de tipo finito, então $M \otimes_{A} N$ também é de tipo finito.

Consideremos as $R$-álgebras $A, B$ e $C$, e os bimódulos ${ }_{B} M_{A}$ e ${ }_{A} N_{C}$. Neste caso, o produto tensorial $M \otimes_{A} N$ (que é um $R$-módulo) tem uma estrutura natural de $(B$ - $C$ )-bimódulo dada por $b(m \otimes n) c:=b m \otimes n c$.

Sejam $f: M_{A} \rightarrow M_{A}^{\prime}$ e $g: N_{A} \rightarrow N_{A}^{\prime}$ morfismos. A aplicação $f \times g: M \times N \rightarrow M^{\prime} \times N$ 
definida por $(f \times g)(m, n):=(f(m), g(n))$ composta com a função tensorial de $M^{\prime} \otimes_{A} N^{\prime}$ é uma aplicação $A$-bilinear de $M \times N$ em $M^{\prime} \otimes_{A} N^{\prime}$. Logo induz uma aplicação $R$-linear denotada por $f \otimes g: M \otimes_{A} N \rightarrow M^{\prime} \otimes_{A} N^{\prime}$ que satisfaz $(f \otimes g)(m \otimes n)=f(m) \otimes g(n)$. Denotaremos por $f \otimes M$ a aplicação $f \otimes i d_{M}$, onde $i d_{M}$ é a aplicação identidade de $M$.

Fixado uma $A$-módulo $M_{A}$, fica definido um funtor covariante $M \otimes_{A}-: \operatorname{Mod} A^{o p} \rightarrow \operatorname{Mod} R$ que associa a cada $A$-módulo ${ }_{A} L$ o $R$-módulo $M \otimes_{A} L$ e a cada morfismo de $A$-módulos $f::_{A} L \rightarrow_{A} N$ associa a aplicação $R$-linear $M \otimes_{A} f: M \otimes_{A} L \rightarrow M \otimes_{A} N$. Da mesma forma podemos definir o funtor covariante $-\otimes_{A} M: \operatorname{Mod} A \rightarrow \operatorname{Mod} R$, para um $A$-módulo à esquerda ${ }_{A} M$.

Se $A$ e $B$ são $R$-álgebras e ${ }_{A} M_{B}$ é um $(A$ - $B$ )-bimódulo então também temos os seguintes funtores covariantes:

$$
\begin{aligned}
& -\otimes_{A} M_{B}: \operatorname{Mod} A \longrightarrow \operatorname{Mod} B \quad{ }_{A} M \otimes_{B}-: \operatorname{Mod} B^{o p} \longrightarrow \operatorname{Mod} A^{o p} \\
& L_{A} \longmapsto L \otimes_{A} M_{B} \quad \mathrm{e} \quad{ }_{B} N \quad \longmapsto{ }_{A} M \otimes_{B} N \\
& f \quad \longmapsto f \otimes_{A} M \quad f \quad \longmapsto \quad M \otimes_{B} f
\end{aligned}
$$

Também podemos considerar as "restrições" desses funtores para módulos de tipo finito, por exemplo $-\otimes_{A} M_{B}: \bmod A \rightarrow \bmod B$.

Esses funtores são aditivos e exatos à direita, isto é, dada uma sequência exata $L \stackrel{f}{\rightarrow} M \stackrel{g}{\rightarrow} N \rightarrow 0$ então a sequência $X \otimes_{A} L \stackrel{X \otimes_{A} f}{\longrightarrow} X \otimes_{A} M \stackrel{X \otimes_{A} g}{\longrightarrow} X \otimes_{A} N \longrightarrow 0$ também é exata. Quando $X$ for um $A$-módulo projetivo então esse funtor é exato. $\mathrm{O}$ mesmo vale para o funtor da forma $-\otimes_{A} X$.

O próximo exemplo tem aqui um esboço de sua demonstração apenas para ilustrar como são construídos os isomorfismos que envolvem produtos tensoriais.

Exemplo 1.9 Seja $M_{A}$ um A-módulo. Então, $M \otimes_{A} A \cong M_{A}$

Prova. Definimos $f: M \times A \rightarrow M$ por $f(m, a):=$ ma. É fácil verificar que $f$ é uma aplicação A-bilinear, então pela definição do produto tensorial, existe $\bar{f}: M \otimes_{A} A \rightarrow M$ R-linear tal que $\bar{f}(m \otimes a)=m a$. Definimos também $g: M \rightarrow M \otimes_{A} A$ por $g(m):=m \otimes 1_{A}$. Temos que $g$ é R-linear e que $\bar{f} g=i d_{M}$ e $g \bar{f}=i d_{M \otimes_{A} N}$. Portanto, $M \otimes_{A} A \cong M_{A}$ como R-módulos. Porém, observamos que $\bar{f}$ e g são também morfismos de A-módulos, logo $M \otimes_{A} A \cong M_{A}$ também como A-módulos.

Mais ainda, o isomorfismo acima é funtorial, ou seja, $-\otimes_{A} A \approx i d_{\text {Mod } A}$. Além desse valem também os seguintes isomorfismos funtoriais: 
1. Se ${ }_{A} M$ é um módulo então $A \otimes_{A} M \cong_{A} M$

2. Se $L_{A},{ }_{A} M_{B}$ e $N_{B}$ são três módulos, então $L \otimes_{A}\left(M \otimes_{B} N\right) \cong\left(L \otimes_{A} M\right) \otimes_{B} N$

3. Seja $\left\{M_{\lambda}\right\}_{\lambda \in \Lambda}$ uma família de $A$-módulos à direita e $\left\{N_{\gamma}\right\}_{\gamma \in \Gamma}$ uma família de $A$-módulos à esquerda. Então, $\left(\bigoplus_{\lambda \in \Lambda} M_{\lambda}\right) \otimes_{A}\left(\bigoplus_{\gamma \in \Gamma} N_{\gamma}\right) \cong \bigoplus_{(\lambda, \gamma) \in \Lambda \times \Gamma}\left(M_{\lambda} \otimes_{A} N_{\gamma}\right)$

Uma consequência de 3 e do Exemplo 1.9 é que se $L_{A}$ e ${ }_{A} M$ são $A$-módulos e $\Lambda$ um conjunto qualquer, então $L \otimes_{A} A^{(\Lambda)} \cong L_{A}^{(\Lambda)}$ e $A^{(\Lambda)} \otimes_{A} M \cong_{A} M^{(\Lambda)}$.

Proposição 1.7 (Teorema da adjunção) Sejam $A$ e $B$ R-álgebras e ${ }_{A} M_{B}$ um (A-B)-bimódulo. Os funtores $-\otimes_{A} M_{B}: \operatorname{Mod} A \rightarrow \operatorname{Mod} B$ e $\operatorname{Hom}_{B}(M,-): \operatorname{Mod} B \rightarrow \operatorname{Mod} A$ são adjuntos. Ou seja, dados os módulos $L_{A}$ e $N_{B}$ existe o seguinte isomorfismo funtorial (em cada variável):

$$
\operatorname{Hom}_{A}\left(L, \operatorname{Hom}_{B}(M, N)\right) \approx \operatorname{Hom}_{B}\left(L \otimes_{A} M, N\right)
$$

O isomorfismo acima é de $R$-módulos. Porém, se considerarmos $C$ e $D$ duas $R$-álgebras de modo que ${ }_{C} L_{A}$ e ${ }_{D} N_{B}$ sejam bimódulos, então o isomorfismo acima é de $(D$ - $C$ )-bimódulos.

Vale também que se ${ }_{B} M_{A}$ é um bimódulo, então os funtores $M \otimes_{A}-:$ Mod $A^{o p} \rightarrow \operatorname{Mod} B^{o p} \mathrm{e}$ $\operatorname{Hom}_{B}(M,-): \operatorname{Mod} B^{o p} \rightarrow \operatorname{Mod} A^{o p}$ são adjuntos.

Proposição 1.8 Seja A uma $R$-álgebra e $\mathcal{I}$ um ideal bilateral de A.

Para todo A-módulo $L_{A}$ existe um isomorfismo funtorial $L \otimes_{A} \frac{A}{\mathcal{I}} \approx \frac{L}{L \mathcal{I}}$ dado por

$$
l \otimes(a+\mathcal{I}) \mapsto l a+L \mathcal{I}
$$

Proposição 1.9 Sejam $A$ e $B$ duas $R$-álgebras, $P_{A}$ um $A$-módulo projetivo de tipo finito, ${ }_{B} M_{A}$ um bimódulo e $N_{B}$ um B-módulo. Existe um isomorfismo funtorial

$$
N \otimes_{B} \operatorname{Hom}_{A}(P, M) \approx \operatorname{Hom}_{A}\left(P, N \otimes_{B} M\right)
$$


dado por $n \otimes f \mapsto \phi \operatorname{com} \phi(p)=n \otimes f(p)$.

\section{4 Álgebra de Caminhos}

\section{Álgebra básica e conexa}

Um elemento $e$ de uma $R$-álgebra é dito idempotente se $e^{2}:=e e=e$. Um idempotente $e$ é dito central se dado $a \in A$ tem-se $e a=a e$. Dois idempotentes $e$ e $\bar{e}$ são ditos ortogonais se $e \bar{e}=\bar{e} e=0$. Um idempotente $e$ é dito primitivo se dados os idempotentes ortogonais $\hat{e}$ e $\check{e} \operatorname{com} e=\hat{e}+\check{e}$ então $\hat{e}=0$ ou $\check{e}=0$.

Uma $R$-álgebra $A$, não nula, é dita conexa (ou indecomponível) se $A=A_{1} \oplus A_{2}$, com $A_{1}$ e $A_{2}$ $R$-álgebras implicar que $A_{1}=0$ ou $A_{2}=0$; ou equivalentemente, se os únicos idempotentes centrais são 0 e $1_{A}$.

Proposição 1.10 Uma $R$-álgebra $A$ é conexa se, e somente se, dados dois A-módulos projetivos indecomponíveis $P$ e $\bar{P}$, existem A-módulos projetivos indecomponíveis $P=P_{0}, P_{1}, \cdots, P_{n}=\bar{P}$ tais que $\operatorname{Hom}_{A}\left(P_{i}, P_{i+1}\right) \neq 0$ ou $\operatorname{Hom}_{A}\left(P_{i+1}, P_{i}\right) \neq 0, \forall i=1, \cdots, n$.

Sejam $A$ uma $R$-álgebra de Artin e $A_{A}=P_{1}^{n_{1}} \oplus \cdots \oplus P_{r}^{n_{r}}$ a decomposição de $A$ em projetivos indecomponíveis de mod $A, \operatorname{com} P_{i} \neq P_{j}$ sempre que $i \neq j$. Dizemos que $A$ é uma álgebra básica se $n_{i}=1, \forall i=1, \ldots r$.

Proposição 1.11 Seja A uma R-álgebra de Artin. Todo A-módulo projetivo indecomponível de tipo finito é isomorfo a um A-módulo da forma eA, onde e é um idempotente.

Proposição 1.12 Seja A uma R-álgebra de Artin, básica e conexa. Então existem idempotentes ortogonais e primitivos $\left\{e_{1}, \ldots, e_{n}\right\}$ tais que $1_{A}=\sum_{i=1}^{n} e_{i}$. Em particular, $A_{A}=e_{1} A \oplus \cdots \oplus e_{n} A$. Tal conjunto é chamado de sistema completo de idempotentes ortogonais e primitivos de $A$.

\section{Álgebra de caminhos}

Ao longo dessa seção vamos considerar $k$ um corpo algebricamente fechado. 
Uma aljava $\Delta$ é uma quádrupla $\left(\Delta_{0}, \Delta_{1}, s, e\right)$ onde $\Delta_{0}$ e $\Delta_{1}$ são conjuntos e $s, e: \Delta_{1} \rightarrow \Delta_{0}$ são funções. Os elementos de $\Delta_{0}$ são chamados de vértices de $\Delta$ e os elementos de $\Delta_{1}$ são chamados de flechas de $\Delta$. Dada uma flecha $\alpha \in \Delta_{1}$, chamamos $s(\alpha)$ de vértice inicial de $\alpha$ e $e(\alpha)$ de vértice final de $\alpha$. Uma aljava $\Delta$ é dita finita quando os conjuntos $\Delta_{0}$ e $\Delta_{1}$ são finitos.

Exemplo 1.10 Podemos representar uma aljava por um diagrama como, $i \underset{\beta}{\stackrel{\alpha}{\longrightarrow}} \stackrel{\gamma}{\gamma} \stackrel{\gamma}{\rightarrow} \bigcap^{\delta}$ por exemplo. Aqui temos $\Delta_{0}=\{1,2,3\}, \Delta_{1}=\{\alpha, \beta, \gamma, \delta\}, s(\alpha)=s(\beta)=1, s(\gamma)=e(\alpha)=e(\beta)=2 e$ $s(\delta)=e(\delta)=e(\gamma)=3$.

Uma subaljava de $\Delta$ é uma aljava $\left(\tilde{\Delta}_{0}, \tilde{\Delta}_{1}, \tilde{s}, \tilde{e}\right)$ de forma que $\tilde{\Delta}_{0} \subseteq \Delta_{0}, \tilde{\Delta}_{1} \subseteq \Delta_{1}, \tilde{s}=\left.s\right|_{\tilde{\Delta}_{1}}$ e $\tilde{e}=\left.e\right|_{\tilde{\Delta}_{1}}$. Uma subaljava é dita plena se a flecha $a \stackrel{\alpha}{\rightarrow} b$ estiver em $\tilde{\Delta}_{1}$ sempre que $a, b \in \tilde{\Delta}_{0}$.

Um caminho $\omega$ em $\Delta$ de comprimento $n>0$ é uma sequência de flechas $\omega=\alpha_{1} \cdots \alpha_{n}$, tal que $e\left(\alpha_{i}\right)=s\left(\alpha_{i+1}\right)$ para $1 \leq i<n$. Por convenção, um caminho de comprimento zero (ou caminho trivial) é um caminho sem flechas associado a um vértice $a \in \Delta_{0}$, que denotamos por $e_{a}$. Para um caminho não trivial $\omega=\alpha_{1} \cdots \alpha_{n}$ definimos o vértice inicial de $\omega$ por $s(\omega):=s\left(\alpha_{1}\right)$ e o vértice final de $\omega$ por $e(\omega):=e\left(\alpha_{n}\right)$. Para um caminho trivial $e_{a}$ definimos $s\left(e_{a}\right)=e\left(e_{a}\right)=a$. Um caminho $\omega$ de comprimento $n \geq 1$ é dito um ciclo orientado quando $s(\omega)=e(\omega)$.

Um passeio de comprimento $n \geq 1$ de $a \in \Delta_{0}$ para $b \in \Delta_{0}$ é uma sequência de flechas $\gamma=\alpha_{1} \cdots \alpha_{n}$ com $a \in\left\{s\left(\alpha_{1}\right), e\left(\alpha_{1}\right)\right\}, b \in\left\{s\left(\alpha_{n}\right), e\left(\alpha_{n}\right)\right\}$ e $\left\{s\left(\alpha_{i}\right), e\left(\alpha_{i}\right)\right\} \cap\left\{s\left(\alpha_{i+1}\right), e\left(\alpha_{i+1}\right)\right\} \neq \emptyset$ para $1 \leq i<n$.

Dado um vértice $a \in \Delta_{0}$, a subaljava plena $\Delta_{a}$ de $\Delta$ formada pelos vértices $b \in \Delta_{0}$ tais que existe um passeio de $a$ para $b$ é chamada de componente conexa de $\Delta$ contendo $a$. Quando $\Delta=\Delta_{a}$ para algum $a$ dizemos que a aljava $\Delta$ é conexa.

Consideremos agora $\Delta$ uma aljava finita. Seja $k \Delta$ o $k$-espaço vetorial cuja base é o conjunto de todos os caminhos de $\Delta$. Definimos em $k \Delta$ o seguinte produto: dados $\gamma$ e $\sigma$ caminhos de $\Delta$, então

- se $e(\gamma) \neq s(\sigma), \gamma \cdot \sigma=0$;

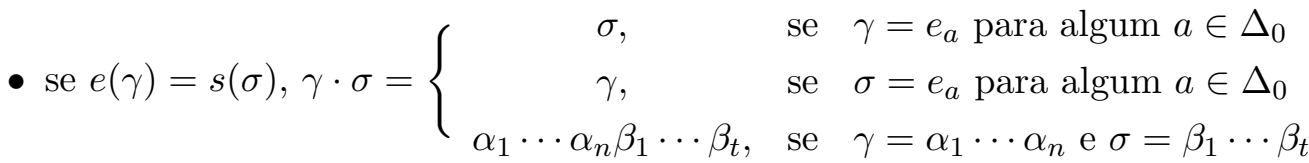


Estendendo esse produto, por linearidade, aos elementos de $k \Delta$ temos que $k \Delta$ é uma $k$-álgebra, a qual chamamos de álgebra de caminhos de $\Delta$.

Exemplo 1.11 Seja $\Delta$ a aljava 1

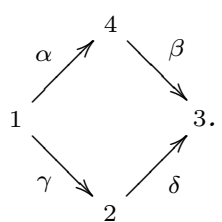

$A$ base de $k \Delta$ como $k$-espaço vetorial é $\left\{e_{1}, e_{2}, e_{3}, e_{4}, \alpha, \beta, \gamma, \delta, \alpha \beta, \gamma \delta\right\}$ e portanto a dimensão de $k \Delta$ é $\operatorname{dim}_{k} k \Delta=10$. Quanto a multiplicação teremos, por exemplo, $\alpha \cdot \beta=\alpha \beta, \alpha \cdot \delta=0, e_{2} \cdot \delta=\delta$, etc.

Denotaremos por $J_{\Delta}$ o ideal de $k \Delta$ gerado pelas flechas de $\Delta$.

Proposição 1.13 Sejam $\Delta$ uma aljava finita com $\Delta_{0}=\{1, \ldots, n\}, k \Delta$ sua álgebra de caminhos e $e_{i}$ o caminho trivial associado ao vértice $i \in \Delta_{0}$. Então:

1. $k \Delta$ é uma álgebra associativa.

2. o conjunto $\left\{e_{i}\right\}_{i \in \Delta_{0}}$ é um sistema completo de idempotentes ortogonais primitivos de $k \Delta$. Em particular, $k \Delta$ tem identidade $1=e_{1}+\cdots+e_{n}$.

3. $k \Delta$ é uma álgebra básica e $k \Delta=e_{1}(k \Delta) \oplus \cdots \oplus e_{n}(k \Delta)$ é a decomposição de $k \Delta$ em módulos indecomponíveis.

4. $k \Delta$ tem dimensão finita se, e somente se, $\Delta$ não possui ciclos orientados.

5. $k \Delta$ é uma álgebra conexa se, e somente se, $\Delta$ é uma aljava conexa.

6. $J_{\Delta}=\operatorname{rad} k \Delta$ se, e somente se, $\Delta$ não possui ciclos orientados.

7. o número de flechas de i para $j$ é igual ao número $\operatorname{dim}_{k}\left(e_{i}\left(\frac{J_{\Delta}}{J_{\Delta}^{2}}\right) e_{j}\right)$.

Um ideal $\mathcal{I}$ de $k \Delta$ é dito admissível se existir $n \geq 2$ tal que $J_{\Delta}^{n} \subseteq \mathcal{I} \subseteq J_{\Delta}^{2}$.

Uma relação $\rho$ em $\Delta$ é uma combinação linear de caminhos de comprimento pelo menos dois, todos com os mesmos vértices iniciais e finais.

Um ideal admissível $\mathcal{I}$ sempre possui um conjunto finito de geradores formado por relações. Por isso chamamos o par $(\Delta, \mathcal{I})$ de aljava com relações. 
Proposição 1.14 Sejam $\Delta$ uma aljava finita, $\mathcal{I}$ um ideal admissível de $k \Delta e \frac{k \Delta}{\mathcal{I}}$ a álgebra quociente. Então:

1. $\frac{k \Delta}{\mathcal{I}}$ tem dimensão finita sobre $k$.

2. o conjunto $\left\{e_{i}+\mathcal{I}\right\}_{i \in \Delta_{0}}$ é um sistema completo de idempotentes ortogonais primitivos de $\frac{k \Delta}{\mathcal{I}}$.

3. $\frac{k \Delta}{\mathcal{I}}$ é uma álgebra básica.

4. $\frac{k \Delta}{\mathcal{I}}$ é uma álgebra conexa se, e somente se, $\Delta$ é uma aljava conexa.

5. $\operatorname{rad} \frac{k \Delta}{\mathcal{I}}=\frac{J_{\Delta}}{\mathcal{I}}$.

Teorema 1.15 Seja $A$ uma k-álgebra básica e de dimensão finita sobre $k$. Existe uma aljava $\Delta_{A} e$ um epimorfismo $\eta_{A}: k \Delta_{A} \rightarrow A$ tal que $\mathcal{I}_{\mathcal{A}}:=$ Nuc $\eta_{\mathcal{A}}$ é um ideal admissivel de $k \Delta_{A}$. Em particular, $A \cong \frac{k \Delta_{A}}{\mathcal{I}_{\mathcal{A}}}$.

O epimorfismo do teorema acima é chamado de uma apresentação de $A$ e a aljava $\Delta_{A}$ é chamada de aljava ordinária de $A$.

\section{Módulos e representações de aljavas}

Sejam $k$ um corpo algebricamente fechado e $\Delta$ uma aljava finita. Uma representação de $\Delta$ é dada por $V=\left(\left(V_{i}\right)_{i \in \Delta_{0}},\left(T_{\alpha}\right)_{\alpha \in \Delta_{1}}\right)$, onde para cada $i \in \Delta_{0}, V_{i}$ é um $k$-espaço vetorial de dimensão finita e para cada $\alpha \in \Delta_{1}, T_{\alpha}$ é uma transformação linear de $V_{s(\alpha)}$ em $V_{e(\alpha)}$.

Exemplo 1.12 Seja $\Delta$ a aljava $1 \underset{\beta}{\stackrel{\alpha}{\longleftarrow}} 2$. Então $k^{2} \underset{T_{\beta}}{\stackrel{T_{\alpha}}{\longleftarrow}} k$ é uma representação de $\Delta$, onde $V_{1}=k^{2}, V_{2}=k, T_{\alpha}=\left[\begin{array}{l}1 \\ 0\end{array}\right]$ e $T_{\beta}=\left[\begin{array}{l}0 \\ 1\end{array}\right]$.

Seja $\omega=\alpha_{1} \alpha_{2} \ldots \alpha_{n}$ um caminho não trivial de $\Delta$. Definimos a transformação linear $T(\omega): V_{s(\omega)} \rightarrow V_{e(\omega)}$ dada pela composta $T_{\alpha_{1}} T_{\alpha_{2}} \cdots T_{\alpha_{n}}$. Estendemos esta definição para uma 
combinação linear de caminhos $\omega=\sum_{i=1}^{t} \lambda_{i} \omega_{i}$, onde $s\left(\omega_{i}\right)=s\left(\omega_{j}\right)$ e $e\left(\omega_{i}\right)=e\left(\omega_{j}\right), \forall i, j \in\{1, \ldots, n\}$, fazendo $T(\omega)=\sum_{i=1}^{t} \lambda_{i} T\left(\omega_{i}\right)$.

Uma representação de $(\Delta, \mathcal{I})$ é uma representação de $\Delta$ de forma que para cada relação $\omega$ de $\mathcal{I}$ tem-se $T(\omega)=0$.

Dadas duas representações $V=\left(\left(V_{i}\right)_{i \in \Delta_{0}},\left(T_{\alpha}\right)_{\alpha \in \Delta_{1}}\right)$ e $W=\left(\left(W_{i}\right)_{i \in \Delta_{0}},\left(S_{\alpha}\right)_{\alpha \in \Delta_{1}}\right)$, um morfismo $\Phi: V \rightarrow W$ é uma família $\left\{\phi_{i}\right\}_{i \in \Delta_{0}}$ de transformações lineares tal que, para cada flecha $i \stackrel{\alpha}{\rightarrow} j \mathrm{o}$ seguinte diagrama é comutativo

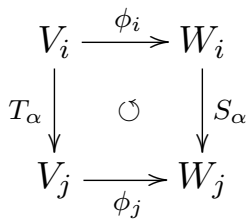

ou seja, $\phi_{j} T_{\alpha}=S_{\alpha} \phi_{i}$. A composta de dois morfismos é definida coordenada a coordenada. Definimos então a categoria mod $(\Delta, \mathcal{I})$ cujos objetos são as representações de $(\Delta, \mathcal{I})$ e os morfismos são os descritos acima.

Teorema 1.16 Seja $A=\frac{k \Delta}{\mathcal{I}}$ onde $\Delta$ é uma aljava finita e $\mathcal{I}$ um ideal admissível de $k \Delta$. Então as categorias $\bmod (\Delta, \mathcal{I})$ e $\bmod A$ são equivalentes.

Pelo teorema acima podemos identificar os $A$-módulos com representações de $(\Delta, \mathcal{I})$. Em especial, as representações que correspondem aos $A$-módulos simples, projetivos indecomponíveis e injetivos indecomponíveis podem ser calculados a partir da aljava ordinária $(\Delta)$ e das relações $(\mathcal{I})$. Para detalhes dessas descrições ver, por exemplo, [8] ou [13].

Exemplo 1.13 Sejam $\Delta$ a aljava $\swarrow_{\gamma}^{\alpha} \swarrow_{\delta}^{\beta}$ e $\mathcal{I}$ o ideal gerado por $\beta \alpha-\delta \gamma$. A seguinte representação de $(\Delta, \mathcal{I})$ dada por ${ }_{0}^{0} \swarrow_{k}^{0} \swarrow_{0}^{0}$ corresponde a um A-módulo simples, que denotamos por 
$S_{3}$. Em muitos casos, representaremos esse módulo escrevendo apenas 00 , onde o " 1 " indica a dimensão do espaço vetorial na "posição 3". 


\section{Capítulo 2}

\section{Extensão cindida por nilpotente}

Neste capítulo define-se o que é uma extensão cindida por nilpotente de uma dada álgebra e algumas propriedades decorrentes desta definição. Para isso consideramos, nessa primeira parte, $R$ um anel artiniano comutativo e $R$-álgebras de Artin. Além disso, os módulos são módulos à direita (caso não se faça menção ao contrário).

\subsection{Propriedades iniciais}

Definição 2.1 Sejam $A$ e $B$ duas R-álgebras e $Q$ um ideal nilpotente de $B$. Dizemos que $B$ é uma extensão cindida de $A$ pelo nilpotente $Q$ (ou mais brevemente extensão cindida por nilpotente de $A$ ) se existir um epimorfismo cindido de álgebras $\pi: B \rightarrow A$ tal que Nuc $\pi=Q$.

Vejamos um primeiro exemplo:

Exemplo 2.1 Consideremos $R=\mathbb{R}$ o corpo dos números reais e as $\mathbb{R}$-álgebras $B=\left(\begin{array}{cc}\mathbb{R} & 0 \\ \mathbb{R} & \mathbb{R}\end{array}\right)$ e $A=\left(\begin{array}{cc}\mathbb{R} & 0 \\ 0 & \mathbb{R}\end{array}\right)$. O conjunto $Q=\left(\begin{array}{ll}0 & 0 \\ \mathbb{R} & 0\end{array}\right)$ é um ideal nilpotente de $B$.

Definimos $\pi: B \longrightarrow A$ por $\left(\begin{array}{ll}x & 0 \\ y & z\end{array}\right) \mapsto\left(\begin{array}{ll}x & 0 \\ 0 & z\end{array}\right)$ que é claramente um epimorfismo com Nuc $\pi=Q$. O morfismo $\sigma: A \rightarrow B$ dado por $\left(\begin{array}{ll}x & 0 \\ 0 & z\end{array}\right) \mapsto\left(\begin{array}{ll}x & 0 \\ 0 & z\end{array}\right)$ é inversa à direita de $\pi$. Portanto, $B$ é uma extensão cindida de A pelo ideal nilpotente $Q$.

A partir da Definição 2.1, já podemos fazer as seguintes observações: 
Observação 2.1 Consideremos $B$ uma extensão cindida de A pelo ideal nilpotente $Q$ e $\pi: B \rightarrow A$ o epimorfismo cindido cujo núcleo é $Q$.

1. Como $\pi$ é um epimorfismo, pelo Teorema 1.3 (do isomorfismo para álgebras), temos $A \cong \frac{B}{Q}$.

2. Como $\pi$ é um epimorfismo cindido, então existe $\sigma: A \rightarrow B$ tal que $\pi \sigma=i d_{A}$, onde $i d_{A}$ é o morfismo identidade da álgebra A.

3. Como $\sigma: A \rightarrow B$ é monomorfismo de álgebras (pois $\pi \sigma=i d_{A}$ ), podemos considerar $A$ uma $R$-subálgebra de $B$ (pois $A \cong \operatorname{Im} \sigma$ que é uma subálgebra de $B$ ).

4. Se $M$ é um A-módulo, podemos dar uma estrutura de $B$-módulo a $M$ definida por

$$
m \cdot b:=m \pi(b), \forall m \in M e \forall b \in B \text {. }
$$

Da mesma forma, se $M$ é um B-módulo, podemos dar uma estrutura de A-módulo a $M$ definida por

$$
m * a:=m \sigma(a), \forall m \in M e \forall a \in A,
$$

onde $\sigma$ é o morfismo do item 2.

Vale o mesmo para módulos à esquerda. Temos, em particular, que B é um A-módulo e $A$ um $B$-módulo. Mais ainda, temos as seguintes estruturas de bimódulos: ${ }_{B} A_{B},{ }_{B} A_{A},{ }_{A} A_{B},{ }_{A} B_{A}$, etc.

5. A sequência exata de (A-A)-bimódulos abaixo cinde.

$$
0 \longrightarrow Q \hookrightarrow B \stackrel{\pi}{\underset{\sigma}{\rightleftarrows}} A \longrightarrow 0
$$

Como A é projetivo (como um (A-A)-bimódulo), basta observar que $\pi$ é um homomorfismo de (A-A)-bimódulos:

sejam a e $a^{\prime}$ em $A$ e $b$ em $B$, temos que

$$
\pi\left(a * b * a^{\prime}\right)=\pi\left(\sigma(a) b \sigma\left(a^{\prime}\right)\right)=\pi \sigma(a) \pi(b) \pi \sigma\left(a^{\prime}\right)=a \pi(b) a^{\prime},
$$

pois $\pi$ é homomorfismo de álgebras e $\pi \sigma=i d_{A}$.

6. Decorre da última observação que o $A$-módulo $B$ pode ser escrito como $B=A \oplus Q$ (soma direta de A-módulos).

7. Como $Q$ é nilpotente, então $Q$ está contido no radical $\operatorname{rad} B$ e, portanto, $\operatorname{rad} A=\frac{\operatorname{rad} B}{Q}$. 
Do item 6 da observação acima temos que $B=A \oplus Q$ como $R$-módulos. Nesse caso o produto da álgebra pode ser escrito por:

Dados $a_{1}+q_{1}, a_{2}+q_{2} \in A \oplus Q$, temos

$$
\left(a_{1}+q_{1}\right)\left(a_{2}+q_{2}\right)=a_{1} a_{2}+\left(a_{1} q_{2}+q_{1} a_{2}+q_{1} q_{2}\right) .
$$

Usaremos, no entanto, a seguinte notação:

$$
\left(a_{1}, q_{1}\right)\left(a_{2}, q_{2}\right)=\left(a_{1} a_{2}, a_{1} q_{2}+q_{1} a_{2}+q_{1} q_{2}\right) .
$$

Observemos ainda que a unidade da álgebra é $\left(1_{A}, 0\right) \in A \oplus Q$.

Usamos essas observações na primeira proposição:

Proposição 2.1 Seja B uma extensão cindida de A pelo nilpotente $Q$ e seja e $\in$ A um idempotente. Então, eBe é uma extensão cindida de eAe por eQe.

Prova. Das observações acima temos $B=A \oplus Q$ como $R$-módulo, e daí podemos escrever também $e B e=e A e \oplus e Q e$. Definimos $\pi: e A e \oplus e Q e \rightarrow e A e$ por $\pi(e a e, e q e)=e a e$, isto é, a projeção de $R$-módulos. Então, $\pi$ é $R$-linear, sobrejetora e para $a, \hat{a} \in A$ e $q, \hat{q} \in Q$ ainda vale:

$\pi((e a e, e q e)(e \hat{a} e, e \hat{q} e))=\pi(e(a e \hat{a}) e, e(a e \hat{q}+q e \hat{a}+q e \hat{q}) e)=e(a e \hat{a}) e=(e a e)(e \hat{a} e)=\pi(e a e, e q e) \pi(e \hat{a} e, e \hat{q} e)$

e

$$
\pi(e, 0)=\pi\left(e 1_{A} e, e 0 e\right)=e 1_{A} e=e,
$$

o que mostra que $\pi$ é um epimorfismo de álgebras.

Para mostrar que cinde, seja $\sigma: e A e \rightarrow e A e \oplus e Q e$ dada por $\sigma(e a e)=(e a e, 0)$. Temos então, para cada $a \in A, \pi \sigma(e a e)=\pi(e a e, 0)=e a e$. Além disso, claramente Nuc $\pi=e Q e$ que é nilpotente pois $e Q e \subseteq Q$ e $Q$ é nilpotente.

\subsection{Categorias $\bmod A$ e $\bmod B$}

Nosso objetivo é identificar propriedades comuns entre as $R$-álgebras $A$ e $B$, quando $B$ é uma extensão cindida por nilpotente de $A$. Para isso, vamos comparar os módulos de tipo finito (finitamente gerados) sobre $A$ e sobre $B$. 


\section{Funtores de mudança de anéis}

Para comparar os módulos de mod $A$ com os módulos em mod $B$ usaremos os funtores:

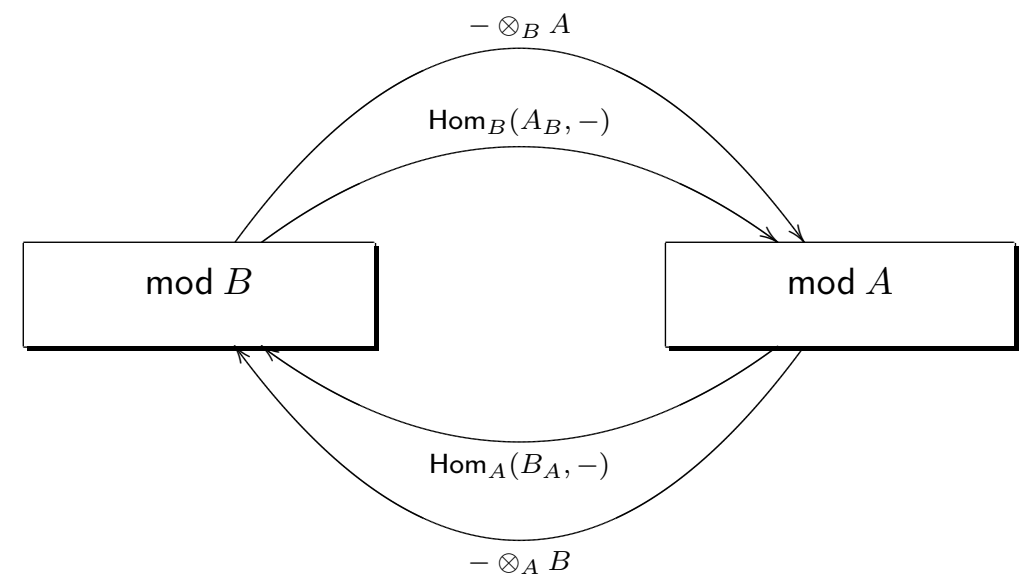

Temos os seguintes isomorfismos funtoriais:

Lema 2.2 Se B é uma extensão cindida por nilpotente de A então:

(a) $-\otimes_{A} B_{B} \otimes_{B} A_{A} \approx i d_{\bmod A}$

(b) $\operatorname{Hom}_{B}\left(A_{B}, \operatorname{Hom}_{A}\left(B_{A},-\right)\right) \approx i d_{\bmod A}$

Prova. Consideremos $\pi: B \rightarrow A$ um epimorfismo cindido tal que Nuc $\pi$ é um ideal nilpotente de $B$, $\sigma: A \rightarrow B$ tal que $\pi \sigma=i d_{A}$ e os produtos $*$ e $\cdot$ como no item 4 da Observação 2.1.

(a) Seja $M_{A}$ um $A$-módulo. Definimos as funções $R$-lineares $\Phi_{M}: M \otimes_{A} B \otimes_{B} A_{A} \rightarrow M_{A}$ por $\Phi_{M}(m \otimes b \otimes a):=m \pi(b) a$ e $\tilde{\Phi}_{M}: M_{A} \rightarrow M \otimes_{A} B \otimes_{B} A_{A}$ por $\tilde{\Phi}_{M}(m):=m \otimes 1_{B} \otimes 1_{A}$.

1. $\Phi_{M}$ e $\tilde{\Phi}_{M}$ são $A$-lineares:

Sejam $a, a^{\prime} \in A, b \in B$ e $m \in M$, então

- $\Phi_{M}\left((m \otimes b \otimes a) a^{\prime}\right)=\Phi_{M}\left(m \otimes b \otimes a a^{\prime}\right)=m \pi(b)\left(a a^{\prime}\right)=(m \pi(b) a) a^{\prime}=\Phi_{M}(m \otimes b \otimes a) a^{\prime}$.

- $\tilde{\Phi}_{M}(m a)=m a \otimes 1_{B} \otimes 1_{A}=m \otimes a * 1_{B} \otimes 1_{A}=m \otimes \sigma(a) \otimes 1_{A}=m \otimes 1_{B} \otimes \sigma(a) \cdot 1_{A}=$ $m \otimes 1_{B} \otimes \pi(\sigma(a)) 1_{A}=m \otimes 1_{B} \otimes a=\left(m \otimes 1_{B} \otimes 1_{A}\right) a=\tilde{\Phi}_{M}(m) a$. 
2. $\Phi_{M} \tilde{\Phi}_{M}=i d_{M}$ e $\tilde{\Phi}_{M} \Phi_{M}=i d_{M_{A} \otimes_{A} B_{B} \otimes_{B} A_{A}}$ :

Sejam $m \in M, a \in A$ e $b \in B$, então

- $\Phi_{M} \tilde{\Phi}_{M}(m)=\Phi_{M}\left(m \otimes 1_{B} \otimes 1_{A}\right)=m \pi\left(1_{B}\right) 1_{A}=m 1_{A} 1_{A}=m=i d_{M}(m)$.

- $\tilde{\Phi}_{M} \Phi_{M}(m \otimes b \otimes a)=\tilde{\Phi}_{M}(m \pi(b) a)=m \pi(b) a \otimes 1_{B} \otimes 1_{A}=m \otimes(\pi(b) a) * 1_{B} \otimes 1_{A}=$ $m \otimes \sigma \pi(b) \sigma(a) \otimes 1_{A}=m \otimes 1_{B} \otimes(\sigma \pi(b) \sigma(a)) \cdot 1_{A}=m \otimes 1_{B} \otimes \pi(\sigma \pi(b) \sigma(a))=$ $m \otimes 1_{B} \otimes \pi(b) a=m \otimes 1_{B} \otimes b \cdot a=m \otimes b \otimes a=i d_{M_{A} \otimes_{A} B_{B} \otimes_{B} A_{A}}(m \otimes b \otimes a)$.

3. $\left\{\Phi_{M}\right\}_{M \in \bmod A}$ é um morfismo funtorial: Sejam $M_{A}$ e $N_{A}$ dois $A$-módulos e $f: M_{A} \rightarrow N_{A}$ um morfismo de $A$-módulos, mostraremos que o diagrama abaixo é comutativo:

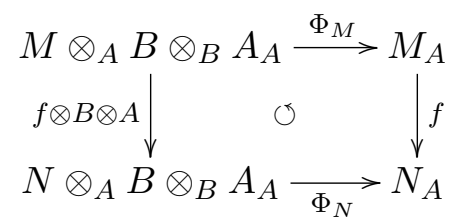

Para todo $m \in M, b \in B$ e $a \in A$ temos que

$\Phi_{N} \circ f \otimes B \otimes A(m \otimes b \otimes a)=\Phi_{N}(f(m) \otimes b \otimes a)=f(m) \pi(b) a=f(m \pi(b) a)=f \circ \Phi_{M}(m \otimes b \otimes a)$.

(b) Para o segundo isomorfismo, definimos as funções $\Psi_{M}: \operatorname{Hom}_{B}\left(A_{B}, \operatorname{Hom}_{A}\left(B_{A}, M_{A}\right)\right) \longrightarrow M_{A}$ por $\Psi_{M}(\phi):=\phi\left(1_{A}\right)\left(1_{B}\right)$ e $\tilde{\Psi}_{M}: M_{A} \longrightarrow \operatorname{Hom}_{B}\left(A_{B}, \operatorname{Hom}_{A}\left(B_{A}, M_{A}\right)\right)$ por $\tilde{\Psi}_{M}(m)(a)(b):=$ $\operatorname{ma\pi }(b)$.

1. $\Psi_{M}$ é $A$-linear:

Sejam $\phi, \psi \in \operatorname{Hom}_{B}\left(A_{B}, \operatorname{Hom}_{A}\left(B_{A}, M_{A}\right)\right)$ e $a \in A$, então

- $\Psi_{M}(\phi+\psi)=\left[(\phi+\psi)\left(1_{A}\right)\right]\left(1_{B}\right)=\left[\phi\left(1_{A}\right)+\psi\left(1_{A}\right)\right]\left(1_{B}\right)=\phi\left(1_{A}\right)\left(1_{B}\right)+\psi\left(1_{A}\right)\left(1_{B}\right)=$ $\Psi_{M}(\phi)+\Psi_{M}(\psi)$

- $\Psi_{M}(\phi a)=\left((\phi a)\left(1_{A}\right)\right)\left(1_{B}\right)=\phi\left(a 1_{A}\right)\left(1_{B}\right)=[\phi(\pi \sigma(a))]\left(1_{B}\right)=\phi\left(1_{A} \cdot \sigma(a)\right)\left(1_{B}\right)=$ $\left[\phi\left(1_{A}\right) \sigma(a)\right]\left(1_{B}\right)=\phi\left(1_{A}\right)\left(1_{B} \sigma(a)\right)=\phi\left(1_{A}\right)\left(1_{B} * a\right)=\left[\phi\left(1_{A}\right)\left(1_{B}\right)\right] a=\Psi_{M}(\phi) a$

2. $\tilde{\Psi}_{M}$ é $A$-linear:

Sejam $m, m^{\prime} \in M_{A}, a, a^{\prime} \in A$ e $b \in B$, então

- $\tilde{\Psi}_{M}\left(m+m^{\prime}\right)(a)(b)=\left(m+m^{\prime}\right) a \pi(b)=\operatorname{ma\pi }(b)+m^{\prime} a \pi(b)=\tilde{\Psi}_{M}(m)(a)(b)+\tilde{\Psi}_{M}\left(m^{\prime}\right)(a)(b)=$ $\left[\tilde{\Psi}_{M}(m)(a)+\tilde{\Psi}_{M}\left(m^{\prime}\right)(a)\right](b)=\left[\tilde{\Psi}_{M}(m)+\tilde{\Psi}_{M}\left(m^{\prime}\right)\right](a)(b)$

- $\tilde{\Psi}_{M}\left(m a^{\prime}\right)(a)(b)=\left(m a^{\prime}\right) a \pi(b)=m\left(a^{\prime} a\right) \pi(b)=\tilde{\Psi}_{M}(m)\left(a^{\prime} a\right)(b)=\left(\tilde{\Psi}_{M}(m) a^{\prime}\right)(a)(b)$ 
3. $\Psi_{M} \tilde{\Psi}_{M}=i d_{M}$ e $\tilde{\Psi}_{M} \Psi_{M}=i d_{\operatorname{Hom}_{B}\left(A_{B}, \operatorname{Hom}_{A}\left(B_{A}, M_{A}\right)\right)}$ :

Sejam $m \in M, a \in A, b \in B$ e $\phi \in \operatorname{Hom}_{B}\left(A_{B}, \operatorname{Hom}_{A}\left(B_{A}, M_{A}\right)\right)$, então

- $\Psi_{M} \tilde{\Psi}_{M}(m)=\tilde{\Psi}_{M}(m)\left(1_{A}\right)\left(1_{B}\right)=m 1_{A} \pi\left(1_{B}\right)=m 1_{A} 1_{A}=m$

- $\tilde{\Psi}_{M}\left(\Psi_{M}(\phi)\right)(a)(b)=\left(\Psi_{M}(\phi)\right) a \pi(b)=\Psi_{M}(\phi a \pi(b))=(\phi a \pi(b))\left(1_{A}\right)\left(1_{B}\right)=$ $=\phi(a \pi(b))\left(1_{B}\right)=\phi(a \cdot b)\left(1_{B}\right)=(\phi(a) b)\left(1_{B}\right)=\phi(a)(b)$

4. $\left\{\Psi_{M}\right\}_{M \in \bmod A}$ é um morfismo funtorial: Sejam $M_{A}$ e $N_{A}$ dois $A$-módulos e $f: M_{A} \rightarrow N_{A}$ um morfismo de $A$-módulos, mostraremos que o diagrama abaixo é comutativo:

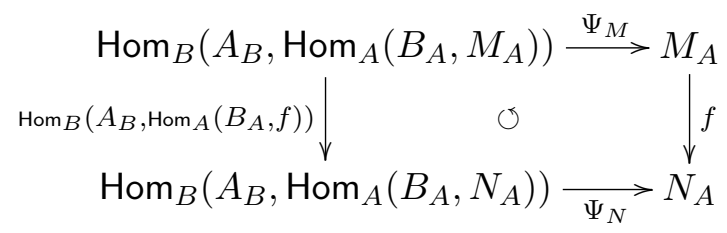

De fato, para todo $\phi \in \operatorname{Hom}_{B}\left(A_{B}, \operatorname{Hom}_{A}\left(B_{A}, M_{A}\right)\right)$ temos que $\Psi_{N} \circ \operatorname{Hom}_{B}\left(A_{B}, \operatorname{Hom}_{A}\left(B_{A}, f\right)\right)(\phi)=\Psi_{N}\left(\operatorname{Hom}_{A}\left(B_{A}, f\right) \circ \phi\right)=\left[\left(\operatorname{Hom}_{A}\left(B_{A}, f\right) \circ \phi\right)\left(1_{A}\right)\right]\left(1_{B}\right)=$ $\left[\operatorname{Hom}_{A}\left(B_{A}, f\right)\left(\phi\left(1_{A}\right)\right)\right]\left(1_{B}\right)=\left(f \circ \phi\left(1_{A}\right)\right)\left(1_{B}\right)=f\left(\phi\left(1_{A}\right)\left(1_{B}\right)\right)=f \circ \Psi_{M}(\phi)$

Observe que não vale $-\otimes_{B} A \otimes_{A} B_{B} \approx i d_{\bmod B}$ e nem $\operatorname{Hom}_{A}\left(B_{A}, \operatorname{Hom}_{B}\left(A_{B},-\right)\right) \approx i d_{\text {mod } B}$. Se fosse esse o caso, teríamos uma equivalência entre as categorias $\bmod A$ e $\bmod B$. Mostraremos que os funtores $-\otimes_{B} A$ não são fiéis, portanto, pela Proposição 1.1 não são equivalências de categorias:

Exemplo 2.2 O funtor $-\otimes_{B} A: \bmod B \rightarrow \bmod A$ não é fiel.

Prova. Sejam $A$ e $B$ duas $R$-álgebras tais que $B$ é uma extensão cindida de $A$ por um ideal nilpotente (não nulo) $Q$. Consideremos a aplicação $-\otimes_{B} A: \operatorname{Hom}_{B}(Q, B) \longrightarrow \operatorname{Hom}_{A}\left(Q \otimes_{B} A, B \otimes_{B} A\right)$. Como $q \otimes a=q 1_{B} \otimes a=1_{B} \otimes q \cdot a=1_{B} \otimes \pi(q) a=0$ para todo $q \in Q$ e para todo $a \in A$, temos $\operatorname{Hom}_{A}\left(Q \otimes_{B} A, B \otimes_{B} A\right)=\{0\}$. Mas o homomorfismo (B-linear) inclusão, $\iota: Q_{B} \longrightarrow B_{B}$, é não nulo, portanto essa aplicação não é injetora.

Exemplo 2.3 O funtor $\operatorname{Hom}_{B}\left(A_{B},-\right): \bmod B \rightarrow \bmod A$ não é fiel.

Prova. Sejam $A$ e $B$ duas $R$-álgebras tais que $B$ é uma extensão cindida de $A$ por um ideal nilpotente (não nulo) $Q$. Consideremos a aplicação 
$\operatorname{Hom}_{B}\left(A_{B},-\right): \operatorname{Hom}_{B}(D Q, D Q) \longrightarrow \operatorname{Hom}_{A}\left(\operatorname{Hom}_{B}(A, D Q), \operatorname{Hom}_{B}(A, D Q)\right)$ onde $D$ é o funtor dual definido no Exemplo 1.8. O morfismo identidade $i d: D Q \rightarrow D Q$ é não nulo. Mostraremos que $\operatorname{Hom}_{A}\left(\operatorname{Hom}_{B}(A, D Q), \operatorname{Hom}_{B}(A, D Q)\right)=0$, o que implicará que $\operatorname{Hom}_{B}\left(A_{B},-\right)$ não é fiel. Pela definição de funtor dual e pelo Teorema da adjunção (1.7), vale a seguinte sequência de isomorfismos:

$$
\operatorname{Hom}_{B}(A, D Q)=\operatorname{Hom}_{B}\left(A, \operatorname{Hom}_{R}(Q, R)\right) \cong \operatorname{Hom}_{R}\left(A \otimes_{B} Q, R\right)=D\left(A \otimes_{B} Q\right) .
$$

Pelo mesmo raciocínio do exemplo anterior, $A \otimes_{B} Q=0$ e portanto $\operatorname{Hom}_{B}(A, D Q)=0$.

Veremos agora uma outra forma de olhar o funtor $-\otimes_{B} A: \bmod B \rightarrow \bmod A$.

Dado um $B$-módulo $M$, observe que $\frac{M}{M Q}$ é anulado por $Q$, logo tem uma estrutura natural de $\frac{B}{Q}$ módulo, dada por $(m+M Q)(b+Q):=m b+M Q$, e consequentemente de $A$-módulo $((m+M Q) \cdot a:=$ $(m+M Q)(\sigma(a)+Q)=m \sigma(a)+M Q)$.

Definimos o funtor $F: \bmod B \rightarrow \bmod A$ associando a cada $B$-módulo $M$, o $A$-módulo $F M:=\frac{M}{M Q}$, e para cada morfismo de $B$-módulos $f: M_{B} \rightarrow N_{B}$, o morfismo de $A$-módulos $F f: \frac{M}{M Q} \rightarrow \frac{N}{N Q}$ dado por $m+M Q \mapsto f(m)+N Q$.

Lembremos que um funtor $G: \bmod B \rightarrow \bmod A$ é dito $R$-linear se para cada par de módulos $\left(X_{B}, Y_{B}\right)$ a aplicação

$$
\begin{array}{ccc}
\operatorname{Hom}_{B}(X, Y) & \rightarrow & \operatorname{Hom}_{A}(G X, G Y) \\
f & \mapsto & F f
\end{array}
$$

é $R$-linear. O funtor $F$ aqui definido é $R$-linear pois para $f, g \in \operatorname{Hom}_{B}(M, N), x \in R$ e $m \in M$, vale $F(f x+g)(m+M Q)=(f x+g)(m)+N Q=(f x)(m)+g(m)+N Q=(f(x m)+N Q)+(g(m)+N Q)=$ $((F f) x+F g)(m+M Q)$.

Além disso, $F$ preserva somas diretas, pois

$$
F\left(\bigoplus M_{i}\right)=\frac{\bigoplus M_{i}}{\left(\bigoplus M_{i}\right) Q} \cong \frac{\bigoplus M_{i}}{\bigoplus M_{i} Q} \cong \bigoplus \frac{M_{i}}{M_{i} Q}=\bigoplus F M_{i}
$$

Proposição 2.3 O funtor $F$ é funtorialmente isomorfo $a-\otimes_{B} A: \bmod B \rightarrow \bmod A$.

Para a demonstração vamos utilizar o Teorema de Watts, que enunciaremos a seguir:

Lema 2.4 (Teorema de Watts) Sejam $A$ e $B$-álgebras e $G: \operatorname{Mod} B \rightarrow \operatorname{Mod} A$ um funtor R-linear. As condições abaixo são equivalentes: 
(i) $G$ é exato à direita e preserva somas diretas.

(ii) Existe um isomorfismo funtorial $G \approx-\otimes_{B} M$, onde $M$ é o A-módulo $G(B)$.

(iii) G admite um adjunto à direita.

Prova. (da Proposição 2.3) Como o funtor $F$ é $R$-linear e preserva somas diretas, basta demontrar que ele é exato à direita, pois pela equivalência (i) $\Leftrightarrow$ (ii) do teorema de Watts teremos $F \approx-\otimes_{B} \frac{B}{B Q}$ e consequentemente $F \approx-\otimes_{B} A$, uma vez que $F\left(B_{B}\right)=\frac{B}{B Q}=\frac{B}{Q} \cong A$.

Seja $L \stackrel{f}{\longrightarrow} M \stackrel{g}{\longrightarrow} N \longrightarrow 0$ uma sequência exata à direita em mod $B$. Mostraremos que a sequência $\frac{L}{L Q} \stackrel{F f}{\longrightarrow} \frac{M}{M Q} \stackrel{F g}{\longrightarrow} \frac{N}{N Q} \longrightarrow 0$ é exata em $\bmod A$.

A aplicação $F g: \frac{M}{M Q} \rightarrow \frac{N}{N Q}$ é sobrejetora pois dado $n+N Q \in \frac{N}{N Q}$, como $g$ é sobrejetora existe $m \in M$ tal que $g(m)=n$, daí $F g(m+M Q)=g(m)+N Q=n+N Q$.

Mostremos também que $\operatorname{Im} F f=$ Nuc $F g$ :

Se $l \in L$, então $(F g)(F f)(l+L Q)=F g(f(l)+M Q)=g(f(l))+N Q=0$ pois $g f=0$. Portanto, $\operatorname{Im} F f \subseteq$ Nuc $F g$. Por outro lado, seja $m \in M$ tal que $F g(m+M Q)=0$, isto é, $g(m)+N Q=0$. Então, $g(m)=\sum n_{i} q_{i} \in N Q$. Para cada $n_{i}$, seja $m_{i} \in M$ de forma que $g\left(m_{i}\right)=n_{i}$ ( $g$ é sobrejetora). Daí $g(m)=\sum n_{i} q_{i}=\sum g\left(m_{i}\right) q_{i}=\sum g\left(m_{i} q_{i}\right)=g\left(\sum m_{i} q_{i}\right)$ pois $g$ é homomorfismo de $B$-módulos e $q_{i} \in Q \subseteq B$. Então,

$m-\sum m_{i} q_{i} \in$ Nuc $g=\operatorname{Im} f$ e portanto, existe $l \in L$ tal que $m-\sum m_{i} q_{i}=f(l)$, ou seja, $m-f(l)=\sum m_{i} q_{i} \in M Q$. Finalmente, $F f(l+M Q)=f(l)+M Q=m+M Q$, o que mostra que $\operatorname{Im} F f \supseteq$ Nuc $F g$.

\subsection{Projetivos, injetivos e conexidade}

Dadas duas $R$-álgebras $A$ e $B$, onde $B$ é extensão cindida por nilpotente de $A$, queremos obter informações de $B$ que sejam herdadas por $A$ e vice-versa. A primeira que iremos analisar é a conexidade dessas álgebras, para isso começaremos estudando a relação entre os módulos projetivos das categorias $\bmod A$ e $\bmod B$. 


\section{Projetivos e Injetivos}

Vamos comparar os módulos projetivos e injetivos em mod $A$ com os projetivos e injetivos em $\bmod B$. Veremos que a cada projetivo (injetivo) em mod $A$ corresponde um projetivo (injetivo) em $\bmod B$ e vice-versa.

Lema 2.5 Seja $M_{B}$ um B-módulo finitamente gerado. Então, $M=0$ se, e somente se, $\frac{M}{M Q}=0$.

Prova. Obviamente $\frac{M}{M Q}=0$ quando $M=0$. Suponhamos $\frac{M}{M Q}=0$, ou seja, $M=M Q$. Como $B$ é uma álgebra de Artin temos que rad $M_{B}=M \operatorname{rad} B$, e como $Q$ é nilpotente temos $Q \subseteq \operatorname{rad} B$. Portanto, $M=M Q \subseteq M \operatorname{rad} B=\operatorname{rad} M_{B}$. Pelo Lema de Nakayama (1.4), se $L \subseteq \operatorname{rad} M$ é submódulo de $M$ e $M+L=M$ então $L=M$. Tomando $L=0$, resulta que $M=0$.

Proposição 2.6 Seja B uma extensão cindida de A pelo ideal nilpotente $Q$, então:

(a) $X_{B} \in \bmod B$ é um $B$-módulo projetivo e indecomponível se, e somente se, existe $P_{A} \in \bmod A$ projetivo e indecomponivel tal que $X \cong P \otimes_{A} B$.

(b) $Y_{B} \in \bmod B$ é um $B$-módulo injetivo e indecomponível se, e somente se, existe $I_{A} \in \bmod A$ injetivo e indecomponivel tal que $Y \cong \operatorname{Hom}_{A}(B, I)$.

Prova.

(a) $\Leftarrow$ Seja $P_{A}$ projetivo e indecomponível, mostraremos que $P \otimes_{A} B_{B}$ é projetivo e indecomponível.

Como $P_{A}$ é projetivo então existem $P_{A}^{\prime}$ e um conjunto $J$ tais que $P \oplus P^{\prime}=A_{A}^{(J)}$. Daí $\left(P \otimes_{A} B\right) \oplus\left(P^{\prime} \otimes_{A} B\right)=A_{A}^{(J)} \otimes_{A} B_{B}=\left(A \otimes_{A} B_{B}\right)^{(J)}=B_{B}^{(J)}$, isto é $P \otimes_{A} B$ é somando do $B$-módulo livre $B_{B}^{(J)}$ e portanto é projetivo.

Suponhamos agora que $P \otimes_{A} B_{B}=M_{B} \oplus N_{B}$. Aplicando o funtor $-\otimes_{B} A$ temos $P_{A} \cong P \otimes_{A} B \otimes_{B} A=\left(M_{B} \oplus N_{B}\right) \otimes_{B} A=\left(M \otimes_{B} A\right) \oplus\left(N \otimes_{B} A\right)$. Pela Proposição 2.3 chegamos a $P_{A} \cong \frac{M}{M Q} \oplus \frac{N}{N Q}$. Como $P_{A}$ é indecomponível temos $\frac{M}{M Q}=0$ ou $\frac{N}{N Q}=0$ e pelo Lema 2.5 segue que $M=0$ ou $N=0$. Portanto, $P \otimes_{A} B_{B}$ é indecomponível. 
$\Rightarrow$ Seja $X_{B}$ projetivo e indecomponível. Então, $B_{B}=\bigoplus_{i} M_{i}$, onde $M_{i}$ são $B$-módulos projetivos e indecomponíveis dois a dois não isomorfos e $X_{B}=M_{i}$ para algum $i$. Da mesma forma $A_{A}=\bigoplus_{j} P_{j} \operatorname{com} P_{j}$ projetivos indecomponíveis, dois a dois não isomorfos. Mas, $B_{B} \cong A \otimes_{A} B_{B} \cong\left(\bigoplus_{j} P_{j}\right) \otimes_{A} B_{B} \cong \bigoplus_{j}\left(P_{j} \otimes_{A} B_{B}\right)$. Logo, $B_{B}=\bigoplus_{i} M_{i} \cong$ $\bigoplus P_{j} \otimes_{A} B_{B}$. Como cada $P_{j} \otimes_{A} B_{B}$ é projetivo e indecomponível, concluímos que $X_{B} \cong$ $\stackrel{j}{P_{j}} \otimes_{A} B_{B}$ para algum $j$.

(b) $\Leftarrow$ Seja $I_{A}$ um $A$-módulo injetivo e indecomponível, mostraremos que $\operatorname{Hom}_{A}(B, I)$ é um $B$ módulo injetivo e indecomponível. Lembremos que se $I_{A}$ é injetivo então o dual $D I$ é um $A^{o p}$-módulo projetivo e se $I_{A}$ é indecomponível então $D I$ é indecomponível. Como $D I$ é um $A^{o p}$-módulo projetivo e indecomponível, com argumento similar ao do item (a), teremos que o $B^{o p}$-módulo $B \otimes_{A} D I$ é projetivo e indecomponível. Daí, o dual deste último $D\left(B \otimes_{A} D I\right)$ é $B$-injetivo. Finalmente, pelo Teorema da adjunção (1.7), a sequência de isomorfismos abaixo nos leva a concluir que $\operatorname{Hom}_{A}(B, I)$ é um $B$-módulo injetivo e indecomponível:

$$
D\left(B \otimes_{A} D I\right)=\operatorname{Hom}_{R}\left(B \otimes_{A} D I, R\right) \cong \operatorname{Hom}_{A}\left(B, \operatorname{Hom}_{R}(D I, R)\right) \cong \operatorname{Hom}_{A}(B, I) .
$$

$\Rightarrow$ Seja $Y_{B}$ um $B$-módulo injetivo e indecomponível. Como $D Y$ é um $B^{o p}$-módulo projetivo e indecomponível, por um argumento similar ao do item (a), existe um $A^{o p}$-módulo projetivo e indecomponível $P$ tal que $D Y \cong B \otimes_{A} P$. Tomando $I_{A}=D P$, temos que $I_{A}$ é injetivo e indecomponível e pelo Teorema da adjunção (1.7) vale a sequência de isomorfismos

$$
\begin{aligned}
Y_{B} & \cong D D Y_{B} \cong D\left(B \otimes_{A} P\right)=\operatorname{Hom}_{R}\left(B \otimes_{A} P, R\right) \\
& \cong \operatorname{Hom}_{A}\left(B, \operatorname{Hom}_{R}(P, R)\right)=\operatorname{Hom}_{A}(B, D P)=\operatorname{Hom}_{A}(B, I) .
\end{aligned}
$$

Se $X$ é um $B$-módulo projetivo em mod $B$, então podemos escrever $X=\bigoplus_{i-1}^{n} X_{i}$, onde cada $X_{i}$ é um $B$-módulo projetivo indecomponível. Pela Proposição 2.6, para cada $i=1, \ldots, n$ existe um 
$A$-módulo projetivo e indecomponível $P_{i}$ tal que $X_{i} \cong P_{i} \otimes_{A} B$. Fazendo $P=\bigoplus_{i-1}^{n} P_{i}$, temos que $P$ é um $A$-módulo projetivo e $X \cong P \otimes_{A} B$. Um raciocínio análogo vale para módulos injetivos.

\section{Conexidade das álgebras}

Com essas informações, em particular sobre módulos projetivos, já podemos enunciar um resultado sobre a conexidade das álgebras envolvidas.

Proposição 2.7 Seja B uma extensão cindida por nilpotente de A. Se A é uma álgebra conexa, então $B$ também é uma álgebra conexa.

Prova. Sejam $X$ e $\hat{X}$ dois $B$-módulos projetivos e indecomponíveis. Pela Proposição 2.6 existem $A$-módulos $P_{A}$ e $\hat{P}_{A}$ tais que $X \cong P \otimes_{A} B$ e $\hat{X} \cong \hat{P} \otimes_{A} B$. Como $A$ é uma álgebra conexa existem $A$-módulos projetivos e indecomponíveis $P=P_{0}, P_{1}, \ldots, P_{n}=\hat{P}$ tais que, para cada $i$,

$$
\operatorname{Hom}_{A}\left(P_{i}, P_{i+1}\right) \neq 0 \text { ou } \operatorname{Hom}_{A}\left(P_{i+1}, P_{i}\right) \neq 0 \text {. }
$$

Daí, $X_{0}=P \otimes_{A} B, X_{1}=P_{1} \otimes_{A} B, \ldots, X_{n}=P_{n} \otimes_{A} B=\hat{P} \otimes_{A} B$ são $B$-módulos projetivos e indecomponíveis e ainda temos que $\operatorname{Hom}_{B}\left(X_{i}, X_{i+1}\right) \neq 0$ ou $\operatorname{Hom}_{B}\left(X_{i+1}, X_{i}\right) \neq 0$ para cada $i$, pois se $f: P_{i} \rightarrow P_{j}$ é não nulo então $f \otimes_{A} B: X_{i} \rightarrow X_{i+1}$ é também não nulo; caso contrário como $\operatorname{Im}\left(f \otimes_{A} B\right)=\operatorname{Im} f \otimes_{A} B$, aplicando o funtor $-\otimes_{B} A$ teríamos $\operatorname{Im} f \cong \operatorname{Im} f \otimes_{A} B \otimes_{B} A=0$. Portanto, $B$ é uma álgebra conexa.

Observação 2.2 A recíproca não vale, como mostra o exemplo a seguir.

Exemplo 2.4 Sejam $A$ e $B$ as $\mathbb{R}$-álgebras do Exemplo 2.1. Já sabemos que $B$ é uma extensão cindida por nilpotente de $A$. Neste caso B é uma álgebra conexa, mas $\left(\begin{array}{ll}1 & 0 \\ 0 & 0\end{array}\right)$ é um idempotente central de A diferente de $\left(\begin{array}{ll}0 & 0 \\ 0 & 0\end{array}\right)$ e $\left(\begin{array}{ll}1 & 0 \\ 0 & 1\end{array}\right)$, ou seja, A não é conexa. 


\section{Capítulo 3}

\section{Aljavas das extensões}

Consideremos agora $k$ um corpo algebricamente fechado e $k$-álgebras dadas por aljavas com relações. Se $B$ é uma extensão cindida de $A$ pelo nilpotente $Q$, qual será a relação entre suas respectivas aljavas? Após um exemplo (que também mostra que a reciproca da Proposição 2.7 não vale) responderemos tal questão, mostraremos uma relação entre as apresentações das álgebras e finalmente caracterizaremos, neste contexto, o ideal nilpotente $Q$. Para isso consideraremos álgebras básicas e com dimensão finita sobre $k$.

Exemplo 3.1 Sejam $\Delta$ a aljava $\overbrace{\delta}^{4} \int_{\delta}^{\beta}$ e $\mathcal{I}$ o ideal (admissivel) de $k \Delta$ gerado pela relação $\alpha \beta-\gamma \delta$. Consideremos $B=\frac{k \Delta}{\mathcal{I}}$ e $Q=<\alpha+\mathcal{I}, \delta+\mathcal{I}>$. Como $\Delta$ é conexa, então $B$ é uma álgebra conexa.

Seja a k-álgebra $A=\frac{B}{Q}$. Temos a seguinte sequência de isomorfismos:

$$
A=\frac{B}{Q}=\frac{k \Delta / \mathcal{I}}{<\alpha+\mathcal{I}, \delta+\mathcal{I}\rangle} \cong \frac{k \Delta}{<\alpha, \delta>} \cong k \hat{\Delta} \text {, onde } \hat{\Delta} \text { é a aljava }{ }_{\gamma}
$$

Nesse caso, B é uma extensão cindida de A pelo ideal nilpotente $Q$, mas $A$ não é conexa, uma vez que $\hat{\Delta}$ não é conexa. 
Antes de relacionarmos as aljavas de $A$ e de $B$, relembraremos a construção de uma apresentação de uma $k$-álgebra.

Observação 3.1 (Apresentação de uma $k$-álgebra) Sejam $C$ uma k-álgebra básica e de dimensão finita sobre $k$ e $\left\{e_{1}, \ldots, e_{n}\right\}$ um conjunto completo de idempotentes ortogonais e primitivos de $C$. Construímos a aljava ordinária de $C$, denotada por $\Delta_{C}$, da seguinte forma:

$O$ conjunto $\left(\Delta_{C}\right)_{0}=\{1, \ldots, n\}$, ou seja, os vértices estão em correspondência biunívoca com o conjunto de idempotentes $\left\{e_{1}, \ldots, e_{n}\right\}$. Dados dois vértices a e $b$, o número de flechas $a \stackrel{\alpha}{\rightarrow} b$ é $\operatorname{dim}_{k}\left(e_{a}\left(\frac{\operatorname{rad} C}{\operatorname{rad}^{2} C}\right) e_{b}\right)$. Mostra-se, em [8] por exemplo, que tal construção independe do conjunto completo de idempotentes ortogonais e primitivos de $C$ escolhido.

Consideremos a álgebra de caminhos $k \Delta_{C}$ e denotemos por $\epsilon_{a}$ o caminho trivial associado ao vértice a. Definimos uma apresentação $\eta_{C}: k \Delta_{C} \rightarrow C$ da seguinte forma:

Escolhemos uma $k$-base $\left\{x_{\alpha}+\operatorname{rad}^{2} C \mid a \stackrel{\alpha}{\rightarrow} b\right\}$ do espaço vetorial $e_{a}\left(\frac{\operatorname{rad} C}{\operatorname{rad}^{2} C}\right) e_{b}$. Definimos então, para cada $i=1, \ldots, n, \eta_{C}\left(\epsilon_{i}\right)=e_{i}$; para cada $\alpha \in\left(\Delta_{C}\right)_{1}, \eta_{C}(\alpha)=x_{\alpha}$ e $\eta_{C}\left(\alpha_{1} \alpha_{2} \ldots \alpha_{t}\right)=x_{\alpha_{1}} x_{\alpha_{2}} \cdots x_{\alpha_{t}}$. Estendemos por linearidade aos demais elementos de $k \Delta$. Mostra-se (em [8] por exemplo) que esse morfismo k-linear é também um morfismo de álgebras, é sobrejetor e $\mathcal{I}_{C}:=$ Nuc $\eta_{C}$ é um ideal admissivel de $k \Delta_{C}$.

Suponhamos que $B$ seja uma extensão cindida de $A$ pelo ideal nilpotente $Q$. Veremos que a aljava de $A$ é uma subaljava de $\Delta_{B}$.

Proposição 3.1 Seja B uma extensão cindida de A pelo ideal nilpotente Q. Então

- $\left(\Delta_{B}\right)_{0}=\left(\Delta_{A}\right)_{0}$.

- Dados os vértices a e b, o conjunto de flechas em $\Delta_{B}$ é o mesmo que em $\Delta_{A}$ mais $\operatorname{dim}_{k}\left(e_{a} \frac{Q}{Q \operatorname{rad} A+(\operatorname{rad} A) Q+Q^{2}} e_{b}\right)$ flechas.

Prova. Seja $\left\{e_{1}, \ldots, e_{n}\right\}$ um conjunto completo de idempotentes ortogonais e primitivos de $B$. Como $e_{i}^{t}=e_{i} \neq 0$ para qualquer $t$, então, como $Q$ é nilpotente, $e_{i} \notin Q$, ou seja, cada $e_{i}+Q$ é não nulo. Daí o conjunto $\left\{e_{1}+Q, \ldots, e_{n}+Q\right\}$ é um conjunto completo de idempotentes ortogonais e primitivos de $\frac{B}{Q}$, pois 
. $\left(e_{i}+Q\right)^{2}=e_{i}^{2}+Q=e_{i}+Q$

. para $i \neq j$ vale que $\left(e_{i}+Q\right)\left(e_{j}+Q\right)=e_{i} e_{j}+Q=0$, pois $e_{i} e_{j}=0$

. Suponhamos que $e_{i}+Q=(e+Q)+(\hat{e}+Q) \operatorname{com} e+Q$ e $\hat{e}+Q$ idempotentes ortogonais. Pelo Teorema do levantamento de idempotentes (ver por exemplo [1], VIII.1.5) podemos supor que $e$ e $\hat{e}$ são idempotentes de $B$. Então, $(e+\operatorname{rad} B)$ e $(\hat{e}+\operatorname{rad} B)$ são idempotentes ortogonais: de $e^{2}+Q=e+Q$ segue que $e^{2}-e \in Q \subseteq \operatorname{rad} B$ e portanto $(e+\operatorname{rad} B)^{2}=e^{2}+\operatorname{rad} B=e+\operatorname{rad} B ;$ e de $(e+Q)(\hat{e}+Q)=0$ segue que $e \hat{e} \in Q \subseteq \operatorname{rad} B$ e portanto $(e+\operatorname{rad} B)(\hat{e}+\operatorname{rad} B)=0$. Além disso, como $e_{i}+Q=e+\hat{e}+Q$, então $e_{i}-(e+\hat{e}) \in Q \subseteq \operatorname{rad} B$ e portanto $e_{i}+\operatorname{rad} B=$ $(e+\operatorname{rad} B)+(\hat{e}+\operatorname{rad} B)$. Segue então que $e \in \operatorname{rad} B$ ou $\hat{e} \in \operatorname{rad} B, \operatorname{pois} e_{i}+\operatorname{rad} B$ é primitivo (ver por exemplo [1], VIII.1.6). Como rad $B$ é nilpotente, então $e=0$ ou $\hat{e}=0$. Logo, $e_{i}+Q$ é primitivo.

$1_{A}=1_{B}+Q=\sum_{i=1}^{n} e_{i}+Q=\sum_{i=1}^{n}\left(e_{i}+Q\right)$.

Portanto, $\left(\Delta_{B}\right)_{0}=\left(\Delta_{A}\right)_{0}$.

Para a segunda parte, sejam $a$ e $b$ vértices, então o número de flechas de $a$ para $b$ em $\left(\Delta_{B}\right)_{1}$ é $\operatorname{dim}_{k}\left(e_{a} \frac{\operatorname{rad} B}{\operatorname{rad}^{2} B} e_{b}\right)$.

Como $k$-espaço vetorial temos que a sequência exata abaixo cinde

$$
0 \longrightarrow Q \hookrightarrow \operatorname{rad} B \underset{\sigma^{\prime}}{\stackrel{\pi^{\prime}}{\rightleftarrows}} \operatorname{rad} A \longrightarrow 0
$$

onde $\pi^{\prime}$ e $\sigma^{\prime}$ são as respectivas restrições de $\pi: B \rightarrow A$ e $\sigma: A \rightarrow B$ aos radicais.

Então $\operatorname{rad} B \cong \operatorname{rad} A \oplus Q$ e portanto,

$$
\operatorname{rad}^{2} B \cong(\operatorname{rad} A \oplus Q)(\operatorname{rad} A \oplus Q)=\operatorname{rad}^{2} A \oplus\left(Q(\operatorname{rad} A)+(\operatorname{rad} A) Q+Q^{2}\right)
$$

Daí, como $\operatorname{rad}^{2} A \subseteq \operatorname{rad} A$ e $Q(\operatorname{rad} A)+(\operatorname{rad} A) Q+Q^{2} \subseteq Q$ temos que

$$
\frac{\operatorname{rad} B}{\operatorname{rad}^{2} B} \cong \frac{\operatorname{rad} A \oplus Q}{\operatorname{rad}^{2} A \oplus\left(Q(\operatorname{rad} A)+(\operatorname{rad} A) Q+Q^{2}\right)} \cong \frac{\operatorname{rad} A}{\operatorname{rad}^{2} A} \oplus \frac{Q}{Q(\operatorname{rad} A)+(\operatorname{rad} A) Q+Q^{2}} .
$$


Finalmente, $\operatorname{dim}_{k}\left(e_{a} \frac{\operatorname{rad} B}{\operatorname{rad}^{2} B} e_{b}\right)=\operatorname{dim}_{k}\left(e_{a} \frac{\operatorname{rad} A}{\operatorname{rad}^{2} A} e_{b}\right)+\operatorname{dim}_{k}\left(e_{a} \frac{Q}{Q(\operatorname{rad} A)+(\operatorname{rad} A) Q+Q^{2}} e_{b}\right)$. Como $\operatorname{dim}_{k}\left(e_{a} \frac{\operatorname{rad} A}{\operatorname{rad}^{2} A} e_{b}\right)$ é o número de flechas de $a$ para $b$ em $\left(\Delta_{A}\right)_{1}$ segue o resultado.

\section{Ideal $Q$}

Antes de enunciarmos a caracterização do ideal $Q$ precisamos da seguinte definição:

Definição 3.1 Sejam $\Delta$ uma aljava, $k \Delta$ a álgebra de caminhos de $\Delta$ e $\mathcal{I}$ um ideal de $k \Delta$. Dizemos que um conjunto $S \subseteq k \Delta$ de geradores de $\mathcal{I}$ é minimal se, para cada $\rho$ em $S$, temos:

(a) Se $\rho$ é um caminho em $\Delta$, então para todo subcaminho próprio $\hat{\rho}$ de $\rho$ temos $\hat{\rho} \notin \mathcal{I}$.

(b) Se $\rho=\sum_{j=1}^{m} \lambda_{j} \omega_{j}$ com $m \geq 2, \lambda_{j} \in k$ não nulos e $\omega_{j} \in \Delta$ caminhos de comprimento positivo todos com o mesmo início e o mesmo fim, então para cada subconjunto próprio $J \subset\{1, \ldots, m\}$ não vazio, temos $\sum_{j \in J} \lambda_{j} \omega_{j} \notin \mathcal{I}$.

De forma análoga define-se conjunto minimal de geradores para um ideal de $\frac{k \Delta}{\mathcal{I}}$, onde $\mathcal{I}$ é um ideal admissivel de $k \Delta$.

Lema 3.2 Seja $\mathcal{I}$ um ideal finitamente gerado de uma $k$-álgebra $k \Delta$, onde $\Delta$ é uma aljava. Então existe um conjunto de geradores minimal para $\mathcal{I}$.

Prova. A partir de um conjunto qualquer $\left\{\rho_{1}, \rho_{2}, \ldots, \rho_{s}\right\}$ de geradores de $\mathcal{I}$, podemos obter um novo conjunto $G=\left\{\epsilon_{a} \rho_{i} \epsilon_{b}: a, b \in \Delta_{0}\right.$ e $\left.1 \leq i \leq s\right\}$ de combinações lineares de caminhos com mesmo início e mesmo fim em $\Delta$ e que ainda é gerador de $Q$. Aqui $\epsilon_{a}$ é o caminho trivial do vértice $a \in \Delta_{0}$. Basta observar que cada elemento do primeiro conjunto de geradores pode ser escrito como $\rho_{j}=\sum_{a, b \in \Delta_{0}} \epsilon_{a} \rho_{j} \epsilon_{b}$

Seja $\sigma=\sum_{j=1}^{m} \lambda_{j} \omega_{j} \in G$ com $m \geq 2$, e suponhamos que $\sigma$ não satisfaz a condição (b) da Definição 3.1. Então existe um subconjunto não vazio próprio $J \subset\{1, \ldots, m\}$ tal que $\hat{\sigma} \in \mathcal{I}$, onde $\hat{\sigma}=\sum_{j \in J} \lambda_{j} \omega_{j}$. Podemos trocar $\sigma$ por $\hat{\sigma}$ e $\sigma-\hat{\sigma}$ no conjunto de geradores de $\mathcal{I}$. Repetindo o processo um número finito 
de vezes chegamos a um novo conjunto $G^{\prime}=\left\{\sigma_{1}, \ldots, \sigma_{n}\right\}$ de geradores de $\mathcal{I}$, onde cada combinação com pelo menos 2 caminhos satisfaz a condição (b).

Suponhamos agora que $\sigma \in G^{\prime}$ é um caminho que não satisfaz a condição (a) da Definição 3.1. Nesse caso, existe um subcaminho $\hat{\sigma}$, próprio, de $\sigma$ tal que $\hat{\sigma} \in \mathcal{I}$. Mas então existem $\omega_{1}$ e $\omega_{2}$ caminhos tais que $\sigma=\omega_{1} \hat{\sigma} \omega_{2}$. Troquemos $\sigma$ por $\hat{\sigma}$ em $G^{\prime}$.

Após um número finito de passos chegamos a um conjunto minimal $S=\left\{\rho_{1}, \ldots, \rho_{t}\right\}$ de geradores de $\mathcal{I}$.

Sejam $\omega$ um caminho e $\alpha$ uma flecha. Denotaremos por $\alpha \mid \omega$ se existirem subcaminhos $\hat{\omega}$ e $\check{\omega}$ de $\omega$, tais que $\omega=\hat{\omega} \alpha \check{\omega}$.

Mostraremos agora que existe uma apresentação $\eta_{B}$ para álgebra $B$ tal que o conjunto das flechas que estão em $\left(\Delta_{B}\right)_{1} \backslash\left(\Delta_{A}\right)_{1}$ gera o ideal $Q$.

Proposição 3.3 Sejam $B$ uma extensão cindida de $A$ pelo ideal nilpotente $Q$ e $\eta_{A}: k \Delta \rightarrow A$ uma apresentação de A. Então, existem uma apresentação $\eta_{B}: k \Delta_{B} \rightarrow B$ e morfismos de álgebras $0 \rightarrow k \Delta_{A} \stackrel{\hat{\sigma}}{\rightarrow} k \Delta_{B}, k \Delta_{B} \stackrel{\hat{\pi}}{\rightarrow} k \Delta_{A} \rightarrow 0$ tais que $\hat{\pi} \hat{\sigma}=i d_{k \Delta_{A}}$ e o seguinte diagrama é comutativo

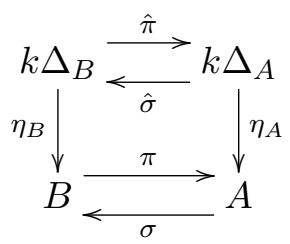

onde $\pi$ é o epimorfismo cindido e $\sigma$ é tal que $\pi \sigma=i d_{A}$.

Prova. Sejam $\pi: B \rightarrow A, \sigma: A \rightarrow B$ morfismos tais que $\pi \sigma=i d_{A}$ e $Q=$ Nuc $\pi$. Podemos identificar $A$ como uma subálgebra de $B$ e $\sigma$ como a inclusão. Pela Proposicao 3.1 podemos considerar $\Delta_{A}$ como uma subaljava de $\Delta_{B}$. A inclusão $\Delta_{A} \hookrightarrow \Delta_{B}$ induz um monomorfismo de álgebras $\hat{\sigma}: k \Delta_{A} \rightarrow k \Delta_{B}$ dado por $\hat{\sigma}\left(\epsilon_{i}\right)=\epsilon_{i}$ para cada $i \in\left(\Delta_{A}\right)_{0}$ e $\hat{\sigma}(\alpha)=\alpha$ para cada $\alpha \in\left(\Delta_{A}\right)_{1}$.

Pela Observação 3.1, o conjunto $X_{i j}:=\left\{\eta_{A}(\alpha)+\operatorname{rad}^{2} A \mid i \stackrel{\alpha}{\rightarrow} j \in\left(\Delta_{A}\right)_{1}\right\}$ é uma $k$-base para $e_{i}\left(\frac{\operatorname{rad} A}{\operatorname{rad}^{2} A}\right) e_{j}$. 
Seja $S:=\left(\Delta_{B}\right)_{1} \backslash\left(\Delta_{A}\right)_{1}$. Pela Proposição 3.1 o número de flechas de $i$ para $j$ em $S$ é

$$
\operatorname{dim}_{k}\left(e_{i} \frac{Q}{Q \operatorname{rad} A+(\operatorname{rad} A) Q+Q^{2}} e_{j}\right)
$$

Para cada flecha $i \stackrel{\beta}{\rightarrow} j$ em $S$ escolhemos $q_{\beta} \in Q$ de modo que o conjunto $Y_{i j}:=\left\{q_{\beta}+\left(Q \operatorname{rad} A+(\operatorname{rad} A) Q+Q^{2}\right) \mid i \stackrel{\beta}{\rightarrow} j \in S\right\}$ seja uma $k$-base para $e_{i} \frac{Q}{Q \operatorname{rad} A+(\operatorname{rad} A) Q+Q^{2}} e_{j}$.

Temos então que $X_{i j} \cup Y_{i j}$ é uma base para $e_{i}\left(\frac{\operatorname{rad}^{2}}{\operatorname{rad}^{2} B}\right) e_{j}$. Definimos uma apresentação para $B$ fazendo $\eta_{B}\left(\epsilon_{i}\right)=e_{i}$ para cada $i \in\left(\Delta_{B}\right)_{0}, \eta_{B}(\alpha)=\eta_{A}(\alpha)$ para cada $\alpha \in\left(\Delta_{A}\right)_{1}$ e $\eta_{B}(\beta)=q_{\beta}$ para cada $\beta \in S$.

Dessa forma temos que $\eta_{B} \hat{\sigma}=\sigma \eta_{A}$ pois $\eta_{B} \hat{\sigma}\left(\epsilon_{i}\right)=\eta_{B}\left(\epsilon_{i}\right)=e_{i}=\sigma \eta_{A}\left(\epsilon_{i}\right)$ e para $\alpha \in\left(\Delta_{A}\right)_{1}$ temos $\eta \hat{\sigma}(\alpha)=\eta_{B}(\alpha)=\eta_{A}(\alpha)=\sigma \eta_{A}(\alpha)$.

Definimos também $\hat{\pi}: k \Delta_{B} \rightarrow k \Delta_{A}$ por $\hat{\pi}\left(\epsilon_{i}\right)=\epsilon_{i}$ para cada $i \in\left(\Delta_{B}\right)_{0}, \hat{\pi}(\alpha)=\alpha$ para cada $\alpha \in\left(\Delta_{A}\right)_{1}$ e $\hat{\pi}(\beta)=0$ para cada $\beta \in S$. Temos, claramente, que $\hat{\pi} \hat{\sigma}=i d_{k \Delta_{A}}$. Além disso, $\eta_{A} \hat{\pi}=\pi \eta_{B}$ pois

. para cada $i \in\left(\Delta_{B}\right)_{0}, \pi \eta_{B}\left(\epsilon_{i}\right)=\pi\left(e_{i}\right)=\pi \sigma\left(e_{i}\right)=e_{i}$ e $\eta_{A} \hat{\pi}\left(\epsilon_{i}\right)=\eta_{A}\left(\epsilon_{i}\right)=e_{i}$;

. para cada $\alpha \in\left(\Delta_{A}\right)_{1}, \pi \eta_{B}(\alpha)=\pi \eta_{A}(\alpha)=\pi \sigma \eta_{A}(\alpha)=\eta_{A}(\alpha)$ e $\eta_{A} \hat{\pi}(\alpha)=\eta_{A}(\alpha)$;

. para cada $\beta \in S, \pi \eta_{B}(\beta)=\pi\left(q_{\beta}\right)=0$ e $\eta_{A} \hat{\pi}(\beta)=\eta_{A}(0)=0$.

Corolário 3.4 Nos termos da Proposição 3.3, sejam $\mathcal{I}_{A}:=$ Nuc $\eta_{A}$ e $\mathcal{I}_{B}:=$ Nuc $\eta_{B}$. Então $\hat{\sigma}\left(\mathcal{I}_{A}\right) \subseteq \mathcal{I}_{B}$.

Prova. Seja $\omega$ em $k \Delta_{A}$ tal que $\eta_{A}(\omega)=0$. Então, como $\hat{\sigma}(\omega)=\omega$ e pela comutatividade do diagrama da proposição anterior, $\eta_{B}(\omega)=\eta_{B}(\hat{\sigma}(\omega))=\sigma \eta_{A}(\omega)=\sigma(0)=0$, ou seja, $\hat{\sigma}(\omega)=\omega \in \mathcal{I}_{B}$.

Corolário 3.5 Seja B uma extensão cindida de A pelo ideal nilpotente Q. Então, existe uma apresentação de $B$ e um subconjunto $S$ de flechas de $\left(\Delta_{B}\right)_{1}$, tais que o ideal $Q$ é gerado pelas classes das flechas de $S$. 
Prova. Consideremos a apresentação de $B$ e os morfismos $\hat{\pi}$ e $\hat{\sigma}$ como na demonstração da Proposição 3.3. Por construção, o núcleo de $\hat{\pi}$ é gerado por $S$. Mostraremos que $\eta_{B}($ Nuc $\hat{\pi})=Q$. Se $\beta \in S$ então $\pi \eta_{B}(\beta)=\pi\left(q_{\beta}\right)=0$, ou seja, $\eta_{B}($ Nuc $\hat{\pi}) \subseteq Q$. Para a outra inclusão, seja $q \in Q$ e $\omega \in k \Delta_{B}$ com $q=\eta_{B}(\omega)$.

Como $\hat{\pi} \hat{\sigma}=i d_{k \Delta_{A}}$ então a sequência de $k$-espaços vetoriais $0 \rightarrow$ Nuc $\hat{\pi} \rightarrow k \Delta_{B} \stackrel{\hat{\pi}}{\rightarrow} k \Delta_{A} \rightarrow 0$ cinde e portanto podemos escrever $\omega=\hat{\omega}+\check{\omega} \operatorname{com} \hat{\omega} \in$ Nuc $\hat{\pi}$ e $\check{\omega} \in k \Delta_{A}$. Mostraremos que $\eta_{B}(\hat{\omega})=q$. Para isso, basta mostrar que $\check{\omega} \in \mathcal{I}_{B}$. Temos que $\eta_{A} \hat{\pi}(\omega)=\pi \eta_{B}(\omega)=\pi(q)=0$, ou seja, $\check{\omega}=\hat{\pi}(\omega) \in \mathcal{I}_{A}$. Pelo Corolário 3.4, $\check{\omega}=\hat{\sigma}(\check{\omega}) \in \mathcal{I}_{B}$.

A pergunta natural é quando que um ideal gerado por classes de flechas define uma extensão cindida por nilpotente. Vejamos o exemplo a seguir.

Exemplo 3.2 Consideremos a mesma aljava $\Delta$ e o mesmo ideal $\mathcal{I}$ do exemplo 3.1, porém agora consideremos $Q^{\prime}=\langle\alpha+\mathcal{I}\rangle$. Nesse caso $Q^{\prime}$ é um ideal gerado pela classe de uma flecha de $\Delta$, mas $B=\frac{k \Delta}{\mathcal{I}}$ não é uma extensão cindida por nilpotente de $A=\frac{B}{Q^{\prime}}$. Se fosse esse o caso, teríamos $A$ uma $k$-subálgebra de $B$, o que não acontece, pois em $A$ temos que $\gamma \delta$ é nulo (pois $\left.\gamma \delta+\mathcal{I}=\alpha \beta+\mathcal{I} \in Q^{\prime}\right)$ e em $B$ é não nulo. Notemos que o conjunto $S=\{\alpha\}$ não tem a propriedade do corolário que segue, isto é, $\alpha \mid \alpha \beta$, mas não existe $\mu \in S$ com $\mu \mid \gamma \delta$.

Corolário 3.6 Nos termos da Proposição 3.3, seja $X$ um conjunto minimal de geradores para $\mathcal{I}_{B}$. Então o conjunto $S$ tem a seguinte propriedade:

$$
\begin{gathered}
\text { "sempre que para } \rho=\sum_{i=1}^{m} \lambda_{i} \omega_{i} \in X \text { existirem } i \in\{1, \ldots, m\} \text { e } \alpha_{i} \in S \\
\text { com } \alpha_{i} \mid \omega_{i} \text {, então para cada } j \in\{1, \ldots, m\} \text { existirá } \alpha_{j} \in S \text { tal que } \alpha_{j} \mid \omega_{j} \text { " }
\end{gathered}
$$

Prova. Seja $\rho=\sum_{i=1}^{m} \lambda_{i} \omega_{i} \in X$ tal que para algum $i$ existe $\alpha_{i} \in S$ com $\alpha_{i} \mid \omega_{i}$. Podemos escrever $\rho=\sum_{i \in I} \lambda_{i} \omega_{i}+\sum_{j \in J} \lambda_{j} \omega_{j}$ com $I, J \subseteq\{1, \ldots, m\}$ de forma que, para cada $i \in I$ existe $\alpha_{i} \in S$ com $\alpha_{i} \mid \omega_{i}$, e para cada $j \in J$ não existe $\alpha \in S \operatorname{com} \alpha \mid \omega_{j}$. Mostraremos que $J=\emptyset$. Suponhamos o contrário, então $\hat{\pi}(\rho)=\sum_{j \in J} \lambda_{j} \omega_{j} \in \mathcal{I}_{A}$, pois $\eta_{A} \hat{\pi}(\rho)=\pi \eta_{B}(\rho)=\pi(0)=0$. Pelo Corolário 3.4, $\sum_{j \in J} \lambda_{j} \omega_{j}=\hat{\sigma}\left(\sum_{j \in J} \lambda_{j} \omega_{j}\right) \in \mathcal{I}_{B}$. Contradição com a minimalidade de $X$. Logo, $J=\emptyset$. 
Mostraremos que a propriedade desse corolário é suficiente para garantir que um ideal gerado por flechas defina uma extensão cindida por nilpotente.

Proposição 3.7 Sejam B uma k-álgebra, $\eta_{B}: k \Delta_{B} \rightarrow B$ uma apresentação de $B$ e $Q$ um ideal de $B$, gerado pela classe das flechas de um conjunto $S$. Então existem uma apresentação de $A=\frac{B}{Q}$, $\eta_{A}: k \Delta_{A} \rightarrow A$ e morfismos de álgebras $0 \rightarrow k \Delta_{A} \stackrel{\hat{\sigma}}{\rightarrow} k \Delta_{B}, k \Delta_{B} \stackrel{\hat{\pi}}{\rightarrow} k \Delta_{A} \rightarrow 0$ tais que $\hat{\pi} \hat{\sigma}=i d_{k \Delta_{A}}$ e o seguinte diagrama de linhas e colunas exatas é comutativo

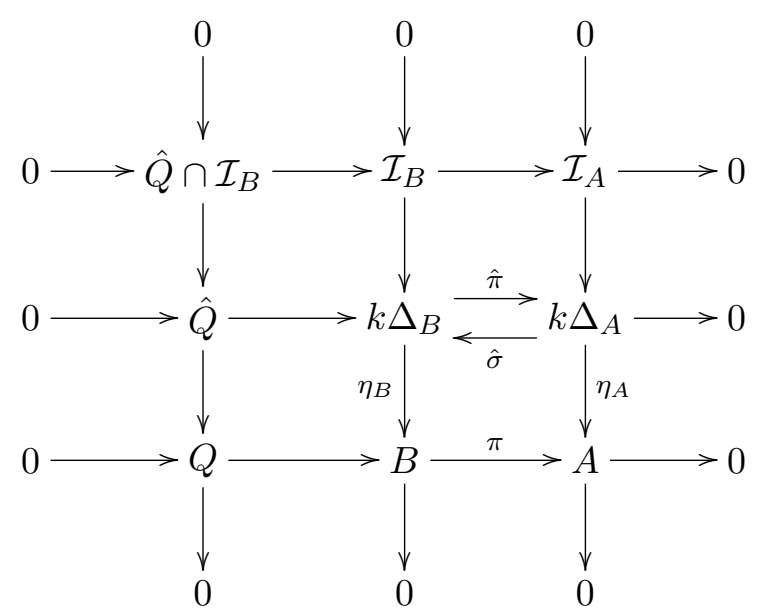

onde $\pi$ é o epimorfismo canônico.

Prova. Pela demonstração da primeira parte da Proposição 3.1 já temos que $\left(\Delta_{A}\right)_{0}=\left(\Delta_{B}\right)_{0}$. Consideremos o conjunto $\Delta_{1}=\left(\Delta_{B}\right)_{1} \backslash S$. Chamando $\Delta_{0}=\left(\Delta_{A}\right)_{0}$, mostraremos que $\Delta_{A}=\Delta$.

Como $\Delta$ é uma subaljava de $\Delta_{B}$ existe um morfismo de álgebras $\hat{\sigma}: k \Delta \rightarrow k \Delta_{B}$ dada por $\hat{\sigma}\left(\epsilon_{i}\right)=\epsilon_{i}$, para cada $i \in \Delta_{0}$ e $\hat{\sigma}(\alpha)=\alpha$ para cada $\alpha \in \Delta_{1}$. Claramente, $\hat{\sigma}$ é um monomorfismo.

Definimos agora $\hat{\pi}: k \Delta_{B} \rightarrow k \Delta$ por

$$
\begin{aligned}
& \hat{\pi}\left(\epsilon_{i}\right)=\epsilon_{i}, \forall i \in\left(\Delta_{B}\right)_{0} \\
& \hat{\pi}(\alpha)=\alpha, \forall \alpha \notin S \\
& \hat{\pi}(\alpha)=0, \forall \alpha \in S
\end{aligned}
$$


e estendendo por linearidade aos demais elementos de $k \Delta_{B}$ teremos que esse morfismo $k$-linear é também um epimorfismo de álgebras, que $\hat{\pi} \hat{\sigma}=1_{k \Delta}$ e que o núcleo é gerado por $S$. Denotemos por $\hat{Q}=\operatorname{Nuc} \hat{\pi}$.

Definimos agora um morfismo $\eta_{A}: k \Delta \rightarrow A$ da seguinte forma: dado $\rho \in k \Delta$, existe $\omega \in k \Delta_{B}$ tal que $\rho=\hat{\pi}(\omega)$. Notemos que $\eta_{B}(\hat{Q})=Q$, daí se $\hat{\pi}(\omega)=\hat{\pi}(\tilde{\omega})$ então $\omega-\tilde{\omega} \in \hat{Q}$ e portanto $\eta_{B}(\omega-\tilde{\omega}) \in Q$, isto é, $\pi \eta_{B}(\omega)=\pi \eta_{B}(\tilde{\omega})$. Podemos então definir $\eta_{A}(\rho):=\pi \eta_{B}(\omega)$.

Então $\eta_{A}$ é um epimorfismo pois $\pi$ e $\eta_{B}$ o são e claramente vale $\pi \eta_{B}=\eta_{A} \hat{\pi}$. Para mostrar que $\eta_{A}$ é uma apresentação de $A$ falta verificar que $\mathcal{I}_{A}:=$ Nuc $\eta_{A}$ é um ideal admissível de $k \Delta$. Daí pela unicidade da aljava ordinária teremos que $\Delta_{A}=\Delta$.

Devemos mostrar que existe $n \geq 2$ tal que $J^{n} \subseteq \mathcal{I}_{A} \subseteq J^{2}$, onde $J$ denota o ideal de $k \Delta$ gerado pelas flechas de $\Delta$. Denotemos também por $J_{\Delta_{B}}$ o ideal de $k \Delta_{B}$ gerado pelas flechas de $\Delta_{B}$.

$\mathcal{I}_{A} \subseteq J^{2}$ : Suponhamos que $\mathcal{I}_{A} \not J^{2}$, e seja $\rho \in \mathcal{I}_{A} \backslash J^{2}$. Podemos escrever $\rho=\sum_{i=1}^{t} \lambda_{i} \alpha_{i}+\gamma$, com $\alpha_{1}, \ldots, \alpha_{t} \in \Delta_{1}, \lambda_{1}, \ldots, \lambda_{t} \in k$ e $\gamma \in J^{2}$. Então $\pi \eta_{B}(\rho)=\eta_{A} \hat{\pi}(\rho)=\eta_{A} \hat{\pi}(\hat{\sigma}(\rho))=\eta_{A}(\rho)=0$, pois $\pi \eta_{B}=\eta_{A} \hat{\pi}, \rho=\hat{\sigma}(\rho) \in k \Delta_{B}, \hat{\pi} \hat{\sigma}=1_{k \Delta}$ e $\rho \in \mathcal{I}_{A}$. Ou seja, $\rho+\mathcal{I}_{B}=\eta_{B}(\rho) \in Q$. Como $Q$ é gerado por classes de flechas de $S$, existem $\beta_{1}, \ldots, \beta_{s} \in S$ e $\delta_{1}, \ldots, \delta_{s} \in k$ tais que $\rho+\mathcal{I}_{B}=\sum_{i=1}^{s} \delta_{i} \beta_{i}+\mathcal{I}_{B}$. Portanto,

$\sum_{i=1}^{t} \lambda_{i} \alpha_{i}+\gamma+\mathcal{I}_{B}=\sum_{i=1}^{s} \delta_{i} \beta_{i}+\mathcal{I}_{B} \Rightarrow$

$\Rightarrow \sum_{i=1}^{t} \lambda_{i} \alpha_{i}+\gamma-\sum_{i=1}^{s} \delta_{i} \beta_{i} \in \mathcal{I}_{B} \subseteq J_{\Delta_{B}}^{2}\left(\right.$ pois $\mathcal{I}_{B}$ é admissível $) \Rightarrow \sum_{i=1}^{t} \lambda_{i} \alpha_{i}-\sum_{i=1}^{s} \delta_{i} \beta_{i} \in J_{\Delta_{B}}^{2}$ $\Rightarrow \sum_{i=1}^{t} \lambda_{i} \alpha_{i}-\sum_{i=1}^{s} \delta_{i} \beta_{i}=0$ (pois $\alpha_{i}$ e $\beta_{i}$ são flechas) $\Rightarrow \sum_{i=1}^{t} \lambda_{i} \alpha_{i}=\sum_{i=1}^{s} \delta_{i} \beta_{i}$.

Contradição, pois cada $\alpha_{i} \in S$ e cada $\beta_{j} \in \Delta_{1}=\left(\Delta_{B}\right)_{1} \backslash S$. Logo, $\mathcal{I}_{A} \subseteq J^{2}$.

$J^{n} \subseteq \mathcal{I}_{A}$ : Como $\mathcal{I}_{B}$ é um ideal admissível de $k \Delta_{B}$, existe $n$ tal que $J_{\Delta_{B}}^{n} \subseteq \mathcal{I}_{B}$. Daí como $\Delta$ é subaljava de $\Delta_{B}$ vale que $J^{n} \subseteq J_{\Delta_{B}}^{n} \subseteq \mathcal{I}_{B}$, ou seja, $\eta_{B}(\rho)=0$ para todo $\rho \in J^{n}$. Finalmente, para todo $\rho \in J^{n}$, temos $\eta_{A}(\rho)=\eta_{A}(\hat{\pi} \hat{\sigma}(\rho))=\pi \eta_{B}(\hat{\sigma}(\rho))=\pi \eta_{B}(\rho)=0$. Logo, $J^{n} \subseteq \mathcal{I}_{A}$. 
Temos então o seguinte diagrama comutativo e de linhas exatas,

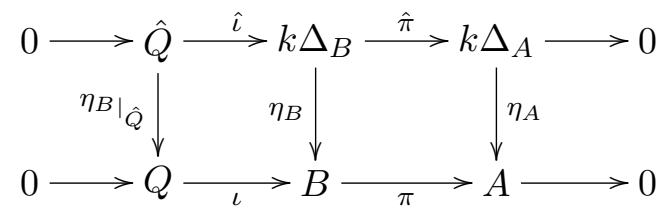

onde $\iota$ e $\hat{\iota}$ são as respectivas inclusões de $Q$ em $B$ e de $\hat{Q}$ em $k \Delta_{B}$.

Pelo Lema da serpente ( [1] por exemplo) chegamos ao diagrama que queremos.

Teorema 3.8 Sejam $\eta_{B}: k \Delta_{B} \rightarrow B$ uma apresentação de $B, X$ um conjunto minimal de geradores de $\mathcal{I}_{B}=$ Nuc $\eta_{B}, Q$ um ideal gerado por classes de flechas de um conjunto $S$, e $\pi: B \rightarrow A=\frac{B}{Q} a$ projeção canônica. Se $S$ tem a seguinte propriedade:

$$
\begin{gathered}
\text { "sempre que para } \rho=\sum_{i=1}^{m} \lambda_{i} \omega_{i} \in X \text { existirem } i \in\{1, \ldots, m\} \text { e } \alpha_{i} \in S \\
\text { com } \alpha_{i} \mid \omega_{i} \text {, então para cada } j \in\{1, \ldots, m\} \text { existirá } \alpha_{j} \in S \text { tal que } \alpha_{j} \mid \omega_{j} \text { " }
\end{gathered}
$$

então $\pi$ é um epimorfismo cindido, ou seja, B é uma extensão cindida de A pelo nilpotente $Q$.

Prova. O ideal $Q$ é nilpotente, isto é, $Q \subseteq \operatorname{rad} B$ pois é gerado por classes de flechas.

Construíremos um morfismo de ágebras $\sigma: A \rightarrow B$ tal que $\pi \sigma=i d_{A}$. Pela Proposição 3.7, existem uma apresentação de $A, \eta_{A}: k \Delta_{A} \rightarrow A$ e morfismos de álgebras $0 \rightarrow k \Delta_{A} \stackrel{\hat{\sigma}}{\rightarrow} k \Delta_{B}$, $k \Delta_{B} \stackrel{\hat{\pi}}{\rightarrow} k \Delta_{A} \rightarrow 0$ tais que $\hat{\pi} \hat{\sigma}=i d_{k \Delta_{A}}$ e $\pi \eta_{B}=\eta_{A} \hat{\pi}$.

Dado $a \in A$ existe $\omega \in k \Delta_{A} \operatorname{com} a=\eta_{A}(\omega)$. Definimos $\sigma(a):=\eta_{B} \hat{\sigma}(\omega)$. Para que $\sigma$ esteja definida é suficiente mostrar que $\hat{\sigma}\left(\mathcal{I}_{A}\right) \subseteq \mathcal{I}_{B}$, onde $\mathcal{I}_{A}=$ Nuc $\eta_{A}$, pois daí se $\eta_{A}(\omega)=\eta_{A}(\tilde{\omega})$, então $\omega-\tilde{\omega} \in \mathcal{I}_{A}$, o que implica que $\hat{\sigma}(\omega-\tilde{\omega}) \in \mathcal{I}_{B}$, ou seja, $\eta_{B} \hat{\sigma}(\omega)=\eta_{B} \hat{\sigma}(\tilde{\omega})$.

Antes de mostrar tal inclusão vejamos que $\sigma$ definida dessa forma é um morfismo de álgebras e é uma inversa à direita de $\pi$ : é um morfismo de álgebras pois $\eta_{A}, \eta_{B}$ e $\hat{\sigma}$ o são. Agora dado $a=\eta_{A}(\omega) \in A$, então $\pi \sigma(a)=\pi \eta_{B} \hat{\sigma}(\omega)=\eta_{A} \hat{\pi} \hat{\sigma}(\omega)=\eta_{A}(\omega)=a$. Portanto, neste caso, $\pi$ é um epimorfismo cindido, ou seja, $B$ é uma extensão cindida de $A$ pelo nilpotente $Q$. 
Voltemos à inclusão $\hat{\sigma}\left(\mathcal{I}_{A}\right) \subseteq \mathcal{I}_{B}$ :

Seja $\rho=\sum_{i=1}^{m} \lambda_{i} \omega_{i} \in X$. Lembremos da demonstração da Proposição 3.7 que $\hat{\pi}(\alpha)=\alpha$ se $\alpha \notin S$ e $\hat{\pi}(\alpha)=0$ se $\alpha \in S$. Se para algum $i, \hat{\pi}\left(\omega_{i}\right)=0$ então existe uma flecha $\alpha_{i} \in S \operatorname{com} \alpha_{i} \mid \omega_{i}$. Daí, pela hipótese, para cada $j$ existe $\alpha_{j} \in S$ com $\alpha_{j} \mid \omega_{j}$, o que implica, nesse caso, que $\hat{\pi}(\rho)=0$. Agora se $\hat{\pi}(\rho) \neq 0$ então $\hat{\pi}\left(\omega_{i}\right) \neq 0$ para algum $i$, ou seja, não existe $\alpha \in S$ com $\alpha \mid \omega_{i}$ e consequentemente, não existe $\alpha \in S$ com $\alpha \mid \omega_{j}$ para todo $j$, o que implica que $\hat{\pi}\left(\omega_{i}\right)=\omega_{i}$, para todo $i$ e portanto $\hat{\pi}(\rho)=\rho$. Ou seja, para todo $\rho \in X$ temos que $\hat{\pi}(\rho)=0$ ou $\hat{\pi}(\rho)=\rho$.

Denotemos por $Y$ um conjunto minimal de geradores de $\mathcal{I}_{A}$. Mostraremos que $\hat{\sigma}(Y) \subseteq \mathcal{I}_{B}$.

Seja $\gamma \in Y$. Como $\hat{\pi}_{\left.\right|_{\mathcal{I}_{B}}}: \mathcal{I}_{B} \rightarrow \mathcal{I}_{A}$ é um epimorfismo (pelo diagrama da Proposição 3.7) existe $\rho \in \mathcal{I}_{B} \operatorname{com} \hat{\pi}(\rho)=\gamma$. Então $\rho=\sum_{i=1}^{m} \lambda_{i} \rho_{i}$, onde $\lambda_{i} \in k$ e cada $\rho_{i}=\rho_{i_{1}} \ldots \rho_{i_{t}}$, com $\rho_{i_{j}} \in X$. Além disso, cada $\hat{\pi}\left(\rho_{i_{j}}\right)=\rho_{i_{j}}$ ou $\hat{\pi}\left(\rho_{i_{j}}\right)=0$ e $\gamma=\hat{\pi}(\rho)=\hat{\pi}\left(\sum_{i=1}^{m} \lambda_{i} \rho_{i}\right)=\sum_{i=1}^{m} \lambda_{i} \hat{\pi}\left(\rho_{i}\right) \operatorname{com} \hat{\pi}\left(\rho_{i}\right)=\hat{\pi}\left(\rho_{i_{1}}\right) \ldots \hat{\pi}\left(\rho_{i_{t}}\right)$.

Como $\gamma$ é não nulo existe $i$ tal que $\hat{\pi}\left(\rho_{i}\right) \neq 0$. Pela minimalidade de $Y$ esse $i$ é único, pois cada $\hat{\pi}\left(\rho_{j}\right) \in \mathcal{I}_{A}$. Daí $\gamma=\lambda_{i} \hat{\pi}\left(\rho_{i}\right)=\lambda_{i} \hat{\pi}\left(\rho_{i_{1}}\right) \ldots \hat{\pi}\left(\rho_{i_{t}}\right)$. Novamente pela minimalidade de $Y$ (e como cada $\left.\hat{\pi}\left(\rho_{i_{j}}\right) \in \mathcal{I}_{A}\right)$, temos que $t=1$ e assim $\gamma=\lambda_{i} \hat{\pi}\left(\rho_{i_{1}}\right)=\lambda_{i} \rho_{i_{1}} \in \mathcal{I}_{B}$. Finalmente, pela definição de $\hat{\sigma}$, $\hat{\sigma}(\gamma)=\gamma \in \mathcal{I}_{B}$

Exemplo 3.3 Agora podemos justificar o Exemplo 3.1. Notemos que $Q$ é gerado pelas classes das flechas de $S=\{\alpha, \delta\}$. Além disso, $\alpha \mid \alpha \beta$ e $\delta \mid \gamma \delta$. Portanto, o epimorfismo canônico $\pi: B \rightarrow \frac{B}{Q}$ cinde. Ou seja, $B$ é uma extensão cindida de $A=\frac{B}{Q}$ pelo ideal $Q$. 


\section{Capítulo 4}

\section{Propriedades homológicas herdadas}

Sejam as $R$-álgebras de Artin $A$ e $B$ tais que $B$ uma extensão cindida de $A$ pelo ideal nilpotente $Q$. Queremos comparar caracteristicas das álgebras $A$ e $B$ envolvendo propriedades homológicas das categorias $\bmod A$ e $\bmod B$. Ao final desse capítulo chegamos a um resultado importante que nos garantirá que se $B$ é uma álgebra hereditária (ou shod) então $A$ também é hereditária (ou shod).

\subsection{Introdução}

Iniciaremos relembrando algumas definições e algumas proposições que serão usadas ao longo do capítulo. Nesta seção, consideraremos $R$ um anel comutativo com unidade e $C$ uma $R$-álgebra de Artin qualquer. Os módulos serão considerados à direita, caso não se faça menção ao contrário.

Definição 4.1 Um epimorfismo de $C$-módulos $f: M \rightarrow N$ é dito supérfluo se, para todo morfismo $h: L \rightarrow M$, a composição $f h: L \rightarrow N$ ser um epimorfismo implicar que $h$ também é um epimorfismo.

Um monomorfismo $f: M \rightarrow N$ é dito essencial se, para todo morfismo $h: N \rightarrow L$, a composição $h f: M \rightarrow L$ ser um monomorfismo implicar que $h$ também é um monomorfismo.

\section{Lema 4.1}

- A composição de epimorfismos supérfluos é ainda supérfluo.

- A composição de monomorfismos essenciais é ainda essencial.

Prova. Faremos a demonstração para epimorfismos supérfluos. O outro caso é análogo. 
Sejam $f: M \rightarrow N$ e $g: N \rightarrow L$ dois epimorfismos supérfluos. Temos que $g f: M \rightarrow L$ é um epimorfismo, falta mostrar que é supérfluo. Seja então, $h: W \rightarrow M$ um morfismo de forma que $(g f) h: W \rightarrow L$ seja um epimorfismo. Como $g$ é supérfluo, temos que $f h$ é um epimorfismo e como $f$ é supérfluo, segue que $h$ é um epimorfismo.

Definição 4.2 Um submódulo $N$ de um $C$-módulo $M$ é dito supérfluo em $M$ se, para todo submódulo $L$ de $M$, a igualdade $N+L=M$ implicar que $L=M$.

Lema 4.2 Um epimorfismo de C-módulos $f: M \rightarrow N$ é supérfluo se, e somente se, Nuc $f$ for um submódulo supérfluo em $M$.

Prova.

$(\Rightarrow)$ Sejam $L$ um submódulo de $M$ tal que Nuc $f+L=M$ e $\iota: L \hookrightarrow M$ a inclusão de $L$ em $M$. Dado $n \in N$ existe $m \in M$ tal que $f(m)=n$, pois $f$ é um epimorfismo. Além disso, podemos escrever $m=x+l \operatorname{com} x \in$ Nuc $f$ e $l \in L$. Finalmente, $n=f(m)=f(x+l)=f(x)+f(l)=f(l)=f h(l)$, isto é, a composição $f \iota$ é um epimorfismo. Como $f$ é supérfluo, temos que $\iota$ é um epimorfismo e portanto um isomorfismo. Logo, $L=M$.

$(\Leftarrow)$ Seja $g: L \rightarrow M$ tal que $f g: L \rightarrow N$ seja um epimorfismo. Mostraremos que $M=\operatorname{Nuc} f+\operatorname{Im} g$ e daí como Nuc $f$ é supérfluo em $M$ resulta que $\operatorname{Im} g=M$, ou seja, que $g$ é um epimorfismo. Obviamente Nuc $f+\operatorname{Im} g \subseteq M$. Por outro lado, dado $m \in M$, como $f g$ é um epimorfismo, existe $l \in L$ tal que $f g(l)=f(m)$. Então $m-g(l) \in$ Nuc $f$ e $m=(m-g(l))+g(l) \in$ Nuc $f+\operatorname{Im} g$.

Agora podemos reescrever o lema de Nakayama (Proposição 1.4) da seguinte forma:

Lema 4.3 (Lema de Nakayama)

Seja $M$ um $C$-módulo de tipo finito. Um submódulo $N$ de $M$ é supérfluo se, e somente se, $N \subseteq \operatorname{rad} M$.

Corolário 4.4 Se $M$ é de tipo finito, então um epimorfismo $f: M \rightarrow N$ é supérfluo se, e somente se, Nuc $f \subseteq \operatorname{rad} M$. 
Definição 4.3 Uma cobertura projetiva de um $C$-módulo $M$ é um par $(P, f)$, onde $P$ é um $C$ módulo projetivo e $f: P \longrightarrow M$ é um epimorfismo supérfluo. Dualmente, uma envolvente injetiva de um $C$-módulo $M$ é um par $(I, g)$, onde I é um $C$-módulo injetivo e $g: M \rightarrow I$ é um monomorfismo essencial.

Mostra-se, em [1] por exemplo, que um módulo de tipo finito sobre uma álgebra de Artin sempre admite uma cobertura projetiva, com $P$ também de tipo finito e uma envolvente injetiva, com $I$ também de tipo finito. Mostraremos agora que tal cobertura é unica a menos de isomorfismo. $\mathrm{O}$ resultado também vale para a envolvente injetiva, mas a demonstração será omitida.

Lema 4.5 Seja $(P, f)$ é uma cobertura projetiva de $M$. Para cada epimorfismo $g: P^{\prime} \longrightarrow M$, com $P^{\prime}$ projetivo, existe um epimorfismo $h: P^{\prime} \longrightarrow P$ tal que $f h=g$.

Prova. Como $P^{\prime}$ é projetivo e $f: P \longrightarrow M$ é um epimorfismo (já que é cobertura projetiva de $M$ ), existe $h: P^{\prime} \longrightarrow P$ tal que $f h=g$, isto é, que faz o seguinte diagrama comutar:

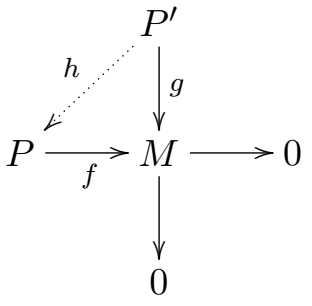

Resta verificar que $h$ é um epimorfismo e isso decorre do fato de $f h$ ser um epimorfismo e de $f$ ser supérfluo.

Proposição 4.6 Sejam $M$ um $C$-módulo de tipo finito, $(P, f)$ e $(\bar{P}, \bar{f})$ coberturas projetivas de $M$. Então existe um isomorfismo $h: \bar{P} \rightarrow P$ tal que $f h=\bar{f}$.

Prova. Como $(P, f)$ é uma cobertura projetiva, pelo Lema 4.5, existe um epimorfismo $h: \bar{P} \longrightarrow P$ tal que $f h=\bar{f}$. Daí, a sequência exata curta $0 \rightarrow$ Nuc $h \rightarrow \bar{P} \stackrel{h}{\rightarrow} P \rightarrow 0$ cinde e portanto $\bar{P} \cong$ Nuc $h \oplus P$. Utilizando novamente o Lema 4.5, trocando $P$ por $\bar{P}$ concluímos que $\bar{P}$ também é um somando de direto de $P$. Portanto, $P \cong \bar{P}$ e Nuc $h=0$, isto é, $h$ é um isomorfismo como queríamos. 
Muitas vezes, por abuso de linguagem, diremos apenas que $P$ é uma cobertura projetiva de $M$, ou ainda, que $f$ é uma cobertura projetiva de $M$. O mesmo para envolvente injetiva.

\section{Definição 4.4}

- Uma apresentação projetiva de um $C$-módulo $M$ é uma sequência exata $P_{1} \stackrel{f_{1}}{\rightarrow} P_{0} \stackrel{f_{0}}{\rightarrow} M \rightarrow 0$ onde $P_{1}$ e $P_{0}$ são $C$-módulos projetivos. Tal apresentação é dita minimal se $f_{0}: P_{0} \rightarrow M e$ $f_{1}: P_{1} \rightarrow$ Nuc $f_{0}$ forem coberturas projetivas.

- Uma apresentação injetiva de um $C$-módulo $M$ é uma sequência exata $0 \rightarrow M \stackrel{g_{0}}{\rightarrow} I_{0} \stackrel{g_{1}}{\rightarrow} I_{1}$, onde $I_{0}$ e $I_{1}$ são C-módulos injetivos. Tal apresentação é dita minimal se $g_{0}: M \rightarrow I_{0}$ e $g_{1}: I_{m} g_{0} \rightarrow I_{1}$ forem envolventes injetivas.

\section{Definição 4.5}

- Uma resolução projetiva de um $C$-módulo $M$ é uma sequência exata

$$
\cdots \rightarrow P_{n} \stackrel{f_{n}}{\longrightarrow} P_{n-1} \longrightarrow \cdots \rightarrow P_{1} \stackrel{f_{1}}{\longrightarrow} P_{0} \stackrel{f_{0}}{\longrightarrow} M \rightarrow 0
$$

onde cada $P_{i}$ é um C-módulos projetivo.

Tal resolução é dita minimal se $f_{0}: P_{0} \rightarrow M$ e $f_{i}: P_{i} \rightarrow N u c f_{i-1}, i=1,2, \ldots$, forem coberturas projetivas.

Tal resolução é dita com comprimento $n$ se $P_{n} \neq 0$ e $P_{i}=0, \forall i>n$.

- Uma resolução injetiva de um C-módulo $M$ é uma sequência exata

$$
0 \rightarrow M \stackrel{g_{0}}{\longrightarrow} I_{0} \stackrel{g_{1}}{\longrightarrow} I_{1} \rightarrow \cdots \rightarrow I_{n-1} \stackrel{g_{n}}{\longrightarrow} I_{n} \rightarrow \cdots
$$

onde cada $I_{i}$ é um $C$-módulo injetivo.

Tal resolução é dita minimal se $g_{0}: M \rightarrow I_{0}$ e $g_{i}: \operatorname{Im}_{i-1} \rightarrow I_{i}, i=1,2, \ldots$, forem envolventes injetivas.

Tal resolução é dita com comprimento $n$ se $I_{n} \neq 0$ e $I_{i}=0, \forall i>n$. 


\section{Definição 4.6}

- Dizemos que a dimensão projetiva de um $C$-módulo $M$ é um inteiro $n$, se esse for o menor inteiro tal que existe uma resolução projetiva de $M$ com comprimento $n$. Denotaremos por dp $M_{C}=n$ (ou $\mathrm{dp} M=n)$.

- Dizemos que a dimensão injetiva de um $C$-módulo $M$ é um inteiro $n$, se esse for o menor inteiro tal que existe uma resolução injetiva de $M$ com comprimento $n$. Denotaremos por di $M_{C}=n$ (ou di $M=n)$.

Definição 4.7 A dimensão global de uma álgebra de Artin $C$, denotado por dim. gl. $C$ é

$$
\sup \left\{\operatorname{dp} M_{C} \mid M_{C} \text { é um } C\right. \text {-módulo\}. }
$$

Mostra-se (ver [1] por exemplo), que se $M$ é de tipo finito (sobre uma álgebra de Artin), então

i) $\operatorname{dp} M_{C}=n$ se, e somente se, a resolução projetiva minimal de $M$ tem comprimento $n$. Analogamente para di $M_{C}=n$;

ii) dim. gl. $C=\sup \left\{\operatorname{dp} M_{C} \mid M_{C}\right.$ é um $C$-módulo de tipo finito $\}=$ $\sup \left\{\mathrm{dp} S_{C} \mid S_{C}\right.$ é um $C$-módulo simples $\}=\sup \left\{\mathrm{dp} M_{C} \mid M_{C}\right.$ é um $C$-módulo indecomponível $\}$.

\section{Transposto}

Precisaremos agora introduzir alguns funtores de $\bmod C$ em $\bmod C^{o p}$. Relembremos, primeiramente, o funtor dual definido no Exemplo 1.8.

Dada uma apresentação projetiva $P_{1} \stackrel{f_{1}}{\rightarrow} P_{0} \stackrel{f_{0}}{\rightarrow} M \rightarrow 0$ de um $C$-módulo $M$, aplicando o funtor dual, chegamos a

$$
0 \longrightarrow D M \stackrel{D f_{0}}{\longrightarrow} D P_{0} \stackrel{D f_{1}}{\longrightarrow} D P_{1}
$$

que é uma apresentação injetiva do $C^{o p}$-módulo $D M$. Mais ainda, se a primeira for minimal a segunda também será. Dualmente, se partirmos de uma apresentação injetiva (minimal) em mod $C$, aplicando o funtor dual, chegaremos a uma apresentação projetiva (minimal) em mod $C^{o p}$. 
Para uma álgebra de Artin $C$, chamamos de $C$-dual ao funtor

$$
(-)^{t}:=\operatorname{Hom}_{C}(-, C): \bmod C \rightarrow \bmod C^{o p} .
$$

Seja $P_{1} \stackrel{f_{1}}{\rightarrow} P_{0} \stackrel{f_{0}}{\longrightarrow} M \rightarrow 0$ uma apresentação projetiva minimal em $\bmod C$. Aplicando o funtor $C$-dual, temos a seguinte sequência exata $0 \rightarrow M^{t} \stackrel{f_{0}^{t}}{\longrightarrow} P_{0}^{t} \stackrel{f_{1}^{t}}{\longrightarrow} P_{1}^{t} \longrightarrow$ Conuc $f_{1}^{t} \rightarrow 0$. Denotaremos Conuc $f_{1}^{t}$ por $\operatorname{Tr}^{C} M$ (ou simplesmente $\operatorname{Tr} M$ ) e chamaremos de transposto de $M$. Desta forma, fica definido um funtor $\operatorname{Tr}: \bmod C \rightarrow \bmod C^{o p}$ que é chamado funtor transposição. Vejamos algumas propriedades desse funtor:

Lema 4.7 ( [11], IV)

(a) $\operatorname{Tr}\left(\bigoplus M_{i}\right) \cong \bigoplus\left(\operatorname{Tr} M_{i}\right)$, onde $M_{i} \in \bmod C$.

(b) $M \in \bmod C$ é projetivo se, e somente se, $\operatorname{Tr} M=0$.

(c) $M \in$ ind $C$ se, e somente se, $\operatorname{Tr} M \in$ ind $C^{o p}$.

(d) Se $M \in$ ind $C$ é não projetivo, então $P_{0}^{t} \stackrel{f_{1}^{t}}{\longrightarrow} P_{1}^{t} \rightarrow \operatorname{Tr} M \rightarrow 0$ é uma apresentação projetiva minimal em $\bmod C^{o p}$.

Também define-se os funtores transladado de Auslander-Reiten (A-R) por $\tau:=D \operatorname{Tr}$ e transladado (de A-R) inverso por $\tau^{-1}:=\operatorname{Tr} D$.

A composição de $D$ com o $C$-dual é o funtor de Nakayama $\nu:=D(-)^{t}$ e $\nu^{-1}:=\operatorname{Hom}_{C}(D C,-)$ é a quase-inversa. 


\section{Lema 4.8}

(a) Seja $P_{1} \stackrel{f_{1}}{\longrightarrow} P_{0} \stackrel{f_{0}}{\longrightarrow} M \rightarrow 0$ uma apresentação projetiva minimal de $M \in \bmod C$. Então existe a sequência exata

$$
0 \rightarrow \tau M \longrightarrow \nu P_{1} \stackrel{\nu f_{1}}{\longrightarrow} \nu P_{0} \stackrel{\nu f_{0}}{\longrightarrow} \nu M \rightarrow 0
$$

(b) Seja $0 \rightarrow N \stackrel{g_{0}}{\longrightarrow} I_{0} \stackrel{g_{1}}{\longrightarrow} I_{1}$ uma apresentação injetiva minimal de $M \in \bmod C$. Então existe a sequência exata

$$
0 \rightarrow \nu^{-1} N \stackrel{\nu^{-1} g_{0}}{\longrightarrow} \nu^{-1} I_{0} \stackrel{\nu^{-1} g_{1}}{\longrightarrow} \nu^{-1} I_{1} \longrightarrow \tau^{-1} N \rightarrow 0
$$

Prova.

(a) Aplicando o funtor $C$-dual à apresentação projetiva minimal $P_{1} \stackrel{f_{1}}{\longrightarrow} P_{0} \stackrel{f_{0}}{\longrightarrow} M \rightarrow 0$ temos, em $\bmod C^{o p}$,

$$
0 \rightarrow M^{t} \stackrel{f_{0}^{t}}{\longrightarrow} P_{0}^{t} \stackrel{f_{1}^{t}}{\longrightarrow} P_{1}^{t} \rightarrow \operatorname{Tr} M \rightarrow 0
$$

Agora aplicando o funtor dual, que é exato:

$$
0 \rightarrow D \operatorname{Tr} M \longrightarrow D P_{1}^{t} \stackrel{D f_{1}^{t}}{\longrightarrow} D P_{0}^{t} \stackrel{D f_{0}^{t}}{\longrightarrow} D M^{t} \rightarrow 0 \mathrm{em} \bmod C
$$

ou seja, $0 \rightarrow \tau M \longrightarrow \nu P_{1} \stackrel{\nu f_{1}}{\longrightarrow} \nu P_{0} \stackrel{\nu f_{0}}{\longrightarrow} \nu M \rightarrow 0$.

(b) Aplicando o funtor dual na apresentação injetiva minimal $0 \rightarrow N \stackrel{g_{0}}{\longrightarrow} I_{0} \stackrel{g_{1}}{\longrightarrow} I_{1}$ temos, em $\bmod C^{o p}$, a seguinte apresentação projetiva minimal:

$$
D I_{1} \stackrel{D g_{1}}{\longrightarrow} D I_{0} \stackrel{D g_{0}}{\longrightarrow} D N \rightarrow 0 .
$$

Agora aplicando o funtor $C^{o p}$-dual, temos

$$
0 \rightarrow(D N)^{t}{\stackrel{\left(D g_{0}\right)^{t}}{\longrightarrow}}^{\longrightarrow}\left(D I_{0}\right)^{t}{\stackrel{\left(D g_{1}\right)^{t}}{\longrightarrow}}^{\longrightarrow}\left(D I_{1}\right)^{t} \longrightarrow \operatorname{Tr}^{C o p} D N \rightarrow 0
$$

Observemos que do Exemplo 1.8 segue os isomorfismos funtoriais para um $C$-módulo $X$ :

$$
(D X)^{t}=\operatorname{Hom}_{C} \text { op }(D X, C) \approx \operatorname{Hom}_{C}(D C, D D X) \approx \operatorname{Hom}_{C}(D C, X)=\nu^{-1} X .
$$

Então: $0 \rightarrow \nu^{-1} N \stackrel{\nu^{-1} g_{0}}{\longrightarrow} \nu^{-1} I_{0} \stackrel{\nu^{-1} g_{1}}{\longrightarrow} \nu^{-1} I_{1} \longrightarrow \tau^{-1} N \rightarrow 0$. 
Proposição 4.9 Seja $C$ uma álgebra de Artin e $M \in \bmod C$. Então,

(a) $\operatorname{dp} M_{C} \leq 1$ se, e somente se, $\operatorname{Hom}_{C}(D C, \tau M)=0$.

(b) di $M_{C} \leq 1$ se, e somente se, $\operatorname{Hom}_{C}\left(\tau^{-1} M, C\right)=0$.

\section{Prova.}

(a) Seja $P_{1} \stackrel{f_{1}}{\longrightarrow} P_{0} \stackrel{f_{0}}{\longrightarrow} M \rightarrow 0$ uma apresentação projetiva minimal de $M$. Pelo Lema 4.8 , existe a sequência exata

$$
0 \rightarrow \tau M \longrightarrow \nu P_{1} \stackrel{\nu f_{1}}{\longrightarrow} \nu P_{0} \stackrel{\nu f_{0}}{\longrightarrow} \nu M \rightarrow 0 .
$$

Aplicando o funtor $\nu^{-1}=\operatorname{Hom}_{C}(D C,-)$ temos o seguinte diagrama comutativo com linhas exatas:

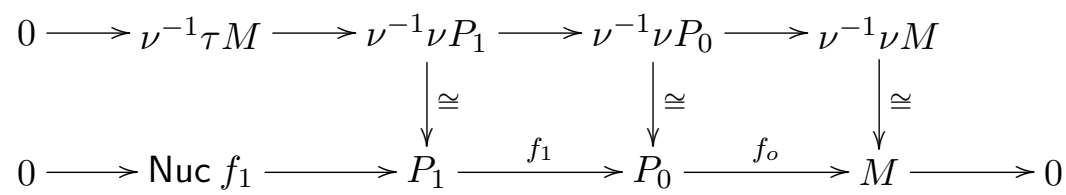

Logo, $\operatorname{Hom}_{C}(D C, \tau M)=\nu^{-1} \tau M \cong$ Nuc $f_{1}$ e Nuc $f_{1}=0$ se, e somente se dp $M \leq 1$.

(b) Seja $0 \rightarrow N \stackrel{g_{0}}{\longrightarrow} I_{0} \stackrel{g_{1}}{\longrightarrow} I_{1}$ uma apresentação injetiva minimal de $N$. Pelo Lema 4.8 existe a sequência exata

$$
0 \rightarrow \nu^{-1} N \stackrel{\nu^{-1} g_{0}}{\longrightarrow} \nu^{-1} I_{0} \stackrel{\nu^{-1} g_{1}}{\longrightarrow} \nu^{-1} I_{1} \longrightarrow \tau^{-1} N \rightarrow 0
$$

Aplicando o funtor $\nu$ temos o diagrama comutativo de linhas exatas:

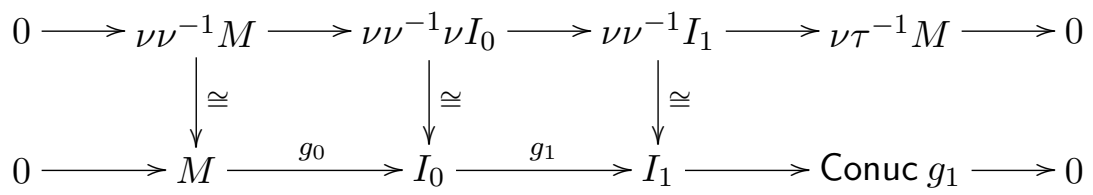

Logo, Conuc $g_{1} \cong \nu \tau^{-1} M=D$ Hom $_{C}\left(\tau^{-1} M, C\right)$. Então, di $M \leq 1$ se, e somente se, Conuc $g_{1}=0$, ou seja, $\operatorname{Hom}_{C}\left(\tau^{-1} M, C\right)=0$. 


\subsection{Propriedades homológicas em $\bmod A$ e em $\bmod B$}

A partir de agora, consideremos $B$ uma extensão cindida de $A$ pelo ideal nilpotente $Q$. Nosso objetivo é comparar as dimensões homólogicas dos módulos em ind $A$ e em ind $B$. Para isso começaremos comparando as respectivas coberturas e apresentações projetivas.

Proposição 4.10 Seja $B$ um extensão cindida de A pelo nilpotente $Q$, então a projeção canônica de B-módulos $p_{M}: M \longrightarrow \frac{M}{M Q}$ é um epimorfismo supérfluo.

Prova. Como Nuc $p_{M}=M Q$ e $M_{B}$ é de tipo finito, basta verificar que $M Q \subseteq \operatorname{rad} M_{B}$. Como $Q$ é nilpotente, já temos $Q \subseteq \operatorname{rad} B$, daí $M Q \subseteq M \operatorname{rad} B=\operatorname{rad} M_{B}$.

Observação 4.1 Seja $M$ é um A-módulo. Olhando $M$ como B-módulo temos que $M Q=0$ pois $Q=$ Nuc $\pi$ (onde $\pi: B \rightarrow A$ é o epimorfismo cindido) e então $m \cdot q=m \pi(q)=0$. Portanto, nesse caso, o epimorfismo canônico $p_{M}: M \longrightarrow \frac{M}{M Q} \cong M$ é na verdade a aplicação identidade de $M_{B}$.

Se considerarmos o B-módulo $M \otimes_{A} B$ teremos, pela Proposição 2.3 e pelo Lema 2.2, que $\frac{M \otimes_{A} B}{\left(M \otimes_{A} B\right) Q} \cong M \otimes_{A} B \otimes_{B} A \cong M$. Neste caso, o epimorfismo canônico $M \otimes_{A} B \rightarrow \frac{M \otimes_{A} B}{\left(M \otimes_{A} B\right) Q}$ pode ser visto como $p_{M \otimes_{A} B}: M \otimes_{A} B \longrightarrow M$, dado por $m \otimes b \mapsto m \cdot b$.

Lembrando que $B \cong A \oplus Q$ como A-módulos, essa última aplicação, olhada como um morfismo de $A$-módulos, pode ainda ser expressa por $m \otimes(a, q) \mapsto m a$, uma vez que $m \cdot(a, q)=m \pi(a, q)=m a$.

Lema 4.11 Seja $f: P \longrightarrow M$ uma cobertura projetiva em $\bmod A$. Então $f \otimes B: P \otimes_{A} B_{B} \rightarrow M \otimes_{A} B_{B}$ é uma cobertura projetiva em $\bmod B$.

Prova. Já sabemos que $P \otimes_{A} B$ é $B$-projetivo, mostraremos que $f \otimes B$ é um epimorfismo supérfluo. De $\operatorname{Im}(f \otimes B)=(\operatorname{Im} f) \otimes_{A} B \cong M \otimes_{A} B$ segue que $f \otimes B$ é um epimorfismo.

Consideremos $P$ e $M$ como $B$-módulos (temos que $f$ é também $B$-linear), $p_{M \otimes_{A} B}: M \otimes_{A} B \rightarrow M$ e $p_{P \otimes_{A} B}: P \otimes_{A} B \rightarrow P$ os epimorfismos canônicos como na Observação 4.1.

Teremos o seguinte diagrama comutativo de $B$-módulos

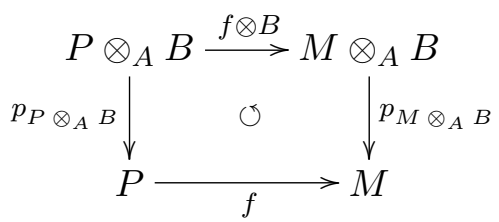


pois $f$ é $B$-linear e então $p_{M \otimes_{A} B}(f \otimes B)(p \otimes b)=p_{M \otimes_{A} B}(f(p) \otimes b)=f(p) \cdot b=f(p \cdot b)=f p_{P \otimes_{A} B}(p \otimes b)$.

Seja agora $h: X \longrightarrow P \otimes_{A} B$ tal que $(f \otimes B) \circ h$ é um epimorfismo. Nesse caso, pela comutatividade do diagrama, $f \circ p_{P \otimes_{A} B} \circ h\left(=p_{M \otimes_{A} B} \circ(f \otimes B) \circ h\right)$ é um epimorfismo. Como $f$ e $p_{P \otimes_{A} B}$ são supérfluos, então $f \circ p_{P \otimes_{A} B}$ também é supérfluo e portanto $h$ é um epimorfismo como queríamos.

Corolário 4.12 Seja $P_{1} \stackrel{f_{1}}{\longrightarrow} P_{0} \stackrel{f_{0}}{\longrightarrow} M \rightarrow 0$ uma apresentação projetiva em $\bmod A$. Então $P_{1} \otimes_{A} B \stackrel{f_{1} \otimes B}{\longrightarrow} P_{0} \otimes_{A} B \stackrel{f_{0} \otimes B}{\longrightarrow} M \otimes_{A} B \rightarrow 0$ é uma apresentação projetiva em $\bmod B$. Mais ainda, se a primeira é minimal, então a segunda também é minimal.

Prova. A primeira parte decorre do fato de que $P \otimes_{A} B$ é projetivo em $\bmod B$ sempre que $P$ é projetivo em mod $A$ e do funtor $-\otimes_{A} B$ ser exato à direita.

Vejamos agora quanto à minimalidade das apresentações. Suponhamos que a primeira é minimal. Pelo Lema 4.11 temos que $P_{0} \otimes_{A} B \stackrel{f_{0} \otimes B}{\longrightarrow} M \otimes_{A} B \rightarrow 0$ é uma cobertura projetiva em mod $B$.

Da mesma forma segue de $P_{1} \stackrel{f_{1}}{\longrightarrow} \operatorname{Im} f_{1} \rightarrow 0$ ser uma cobertura projetiva em mod $A$, que $P_{1} \otimes_{A} B \stackrel{f_{1} \otimes B}{\longrightarrow} \operatorname{Im} f_{1} \otimes_{A} B \rightarrow 0$ é uma cobertura projetiva em mod $B$.

O resultado segue de $\operatorname{Im} f_{1} \otimes_{A} B=\operatorname{Im}\left(f_{1} \otimes_{A} B\right)=\operatorname{Nuc}\left(f_{0} \otimes B\right)$.

Proposição 4.13 Seja $f: P \longrightarrow M$ uma cobertura projetiva em mod A. Então a composição $p_{M \otimes_{A} B}(f \otimes B): P \otimes_{A} B \longrightarrow M$ é uma cobertura projetiva em $\bmod B$.

Prova. Segue da Proposição 4.10 que $p_{M \otimes_{A} B}$ é um $B$-epimorfismo supérfluo e do Lema 4.11 que $f \otimes B$ é um $B$-epimorfismo supérfluo. A composição também é um $B$-epimorfismo supérfluo. $\mathrm{E} P \otimes_{A} B$ é $B$-projetivo porque $P$ é $A$-projetivo.

Corolário 4.14 Seja $M_{A} \in \bmod A$, então dp $M_{B}=0 \Rightarrow \mathrm{dp} M_{A}=0$.

Prova. Seja $f: P \rightarrow M_{A}$ uma cobertura projetiva em mod $A$. Pela proposição anterior $p_{M \otimes_{A} B}(f \otimes B): P \otimes_{A} B \rightarrow M$ é uma cobertura projetiva em $\bmod B$. Como dp $M_{B}=0$ então tal cobertura é um isomorfismo. Temos que Nuc $f \otimes_{A} B \subseteq \operatorname{Nuc} p_{M \otimes_{A} B}(f \otimes B)$ e portanto Nuc $f \otimes_{A} B=0$. Aplicando o funtor $-\otimes_{B} A$ chegamos a Nuc $f=0$ e portanto $f$ é um isomorfismo, isto é, dp $M_{A}=0$. 
Proposição 4.15 Seja $M \in \bmod A$. Se dp $M_{B} \leq 1$ então dp $\left(M \otimes_{A} B\right)_{B} \leq 1$.

Prova. Seja $P_{1} \stackrel{f_{1}}{\longrightarrow} P_{0} \stackrel{f_{0}}{\longrightarrow} M \rightarrow 0$ uma apresentação projetiva minimal em mod $A$. Pelo Corolário 4.12, $P_{1} \otimes_{A} B \stackrel{f_{1} \otimes B}{\longrightarrow} P_{0} \otimes_{A} B \stackrel{f_{0} \otimes B}{\longrightarrow} M \otimes_{A} B \rightarrow 0$ é uma apresentação projetiva minimal em mod $B$. Mostraremos que essa apresentação é na verdade uma resolução projetiva minimal, de onde seguirá que dp $\left(M \otimes_{A} B\right)_{B} \leq 1$.

Seja $0 \rightarrow \widetilde{P}_{1} \stackrel{\widetilde{f}_{1}}{\longrightarrow} \widetilde{P}_{0} \stackrel{\widetilde{f}_{0}}{\longrightarrow} M_{B} \rightarrow 0$ uma resolução projetiva minimal em $\bmod B\left(\mathrm{dp} M_{B} \leq 1\right)$. Pela proposição anterior, temos que $P_{0} \otimes_{A} B$ é cobertura projetiva de $M_{B}$, logo $\widetilde{P}_{0} \cong P_{0} \otimes_{A} B$.

Consideremos $\mathrm{p}:=p_{M \otimes B}$ o epimorfismo canônico da Observação 4.1. Observe que $\operatorname{Im}\left(f_{1} \otimes B\right)=$ $\operatorname{Nuc}\left(f_{0} \otimes B\right) \subseteq \operatorname{Nuc}\left(\mathrm{p}\left(f_{0} \otimes B\right)\right)$ e como $\widetilde{P}_{1} \rightarrow \operatorname{Nuc}\left(\mathrm{p}\left(f_{0} \otimes B\right)\right) \rightarrow 0$ é cobertura projetiva, então existe um morfismo $P_{1} \otimes_{A} B \rightarrow \widetilde{P}_{1}$ que faz o seguinte diagrama de linhas exatas comutar:

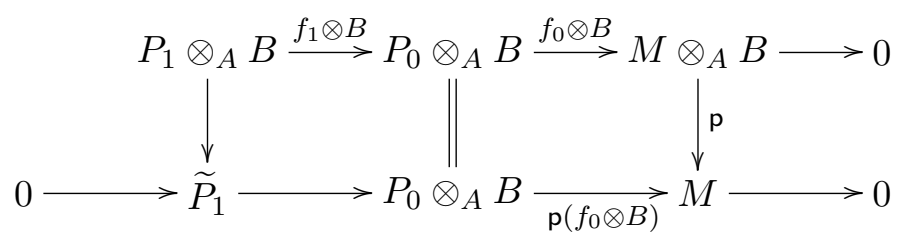

Determinação de $\widetilde{P}_{1}$ :

Consideremos a sequência exata inferior, do diagrama acima, olhada como uma sequência de $A$ módulos. Observemos que, nesse caso, $P_{0} \otimes_{A} B \cong P_{0} \otimes_{A}(A \oplus Q) \cong\left(P_{0} \otimes_{A} A\right) \oplus\left(P_{0} \otimes_{A} Q\right) \cong$ $P_{0} \oplus\left(P_{0} \otimes_{A} Q\right)$ e da mesma forma $M \otimes_{A} B \cong M \oplus\left(M \otimes_{A} Q\right)$.

Dado $(m, \hat{m} \otimes q) \in M \oplus\left(M \otimes_{A} Q\right)$ temos

$$
(m, \hat{m} \otimes q)=(m, m \otimes 0)+(\hat{m} .0, \hat{m} \otimes q) \stackrel{\sim}{\longrightarrow} m \otimes\left(1_{A}, 0\right)+\hat{m} \otimes(0, q) \stackrel{\mathrm{p}}{\longrightarrow} m 1_{A}+\hat{m} 0=m
$$

e portanto, como aplicação $A$-linear, podemos escrever $\mathrm{p}=\left[\begin{array}{ll}1 & 0\end{array}\right]$.

Analogamente, dado $(p, \hat{p} \otimes q) \in P_{0} \oplus\left(P_{0} \otimes_{A} Q\right)$, temos

$$
\begin{gathered}
(p, \hat{p} \otimes q) \stackrel{\sim}{\longrightarrow} p \otimes\left(1_{A}, 0\right)+\hat{p} \otimes(0, q) \stackrel{f_{0} \otimes B}{\longrightarrow} f_{0}(p) \otimes\left(1_{A}, 0\right)+f_{0}(\hat{p}) \otimes(0, q) \stackrel{\sim}{\longrightarrow} \\
\left(f_{0}(p), f_{0}(p) \otimes 0\right)+\left(f_{0}(\hat{p}) 0, f_{0}(\hat{p}) \otimes q\right)=\left(f_{0}(p), 0\right)+\left(0, f_{0}(\hat{p}) \otimes q\right)=\left(f_{0}(p), f_{0}(\hat{p}) \otimes q\right)
\end{gathered}
$$


e portanto, como aplicação $A$-linear, $f_{0} \otimes B=\left[\begin{array}{cc}f_{0} & 0 \\ 0 & f_{0} \otimes Q\end{array}\right]$.

Logo, $\mathrm{p}\left(f_{0} \otimes B\right)=\left[\begin{array}{ll}1 & 0\end{array}\right]\left[\begin{array}{cc}f_{0} & 0 \\ 0 & f_{0} \otimes Q\end{array}\right]=\left[\begin{array}{cc}f_{0} & 0\end{array}\right]$ e então $\widetilde{P}_{1} \cong \operatorname{Nuc}\left[\begin{array}{cc}f_{0} & 0\end{array}\right]$.

Mas, se $p \in P_{0}$ e $t \in P_{0} \otimes_{A} Q$, então $(p, t) \in \operatorname{Nuc}\left[\begin{array}{ll}f_{0} & 0\end{array}\right] \Longleftrightarrow f_{0}(p)=0$ e assim,

$\widetilde{P}_{1} \cong$ Nuc $f_{0} \oplus\left(P_{0} \otimes_{A} Q\right)$.

Como $P_{1}$ é cobertura projetiva de Nuc $f_{0}$ em $\bmod A$ então $P_{1} \otimes_{A} B$ é cobertura projetiva de Nuc $f_{0}$ em $\bmod B$. Seja $P$ a cobertura projetiva de $P_{0} \otimes_{A} Q$ em $\bmod A$, então $P \otimes_{A} B$ é cobertura de $P_{0} \otimes_{A} Q$ em mod $B$. Denotemos por $g: P \otimes_{A} B \longrightarrow P_{0} \otimes_{A} Q$ tal cobertura.

Como $P_{0}$ é projetivo, então $P_{0} \otimes_{A}$ - é exato e aplicando tal funtor em ${ }_{A} Q_{B} \hookrightarrow_{A} B_{B}$ temos a inclusão de $B$-módulos $P_{0} \otimes_{A} Q \stackrel{\iota}{\hookrightarrow} P_{0} \otimes_{A} B$. Chamemos de $\widetilde{f}$ a composição de $g$ com a inclusão $\iota$.

Consideremos agora o seguinte diagrama comutativo com linhas exatas:

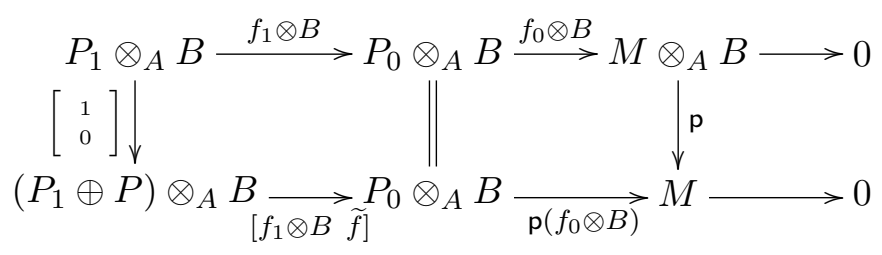

Mostraremos que existe um somando $P^{\prime}$ de $P$ tal que o diagrama de linhas exatas a seguir é comutativo e a linha inferior é uma resolução projetiva minimal para $M_{B}$ :

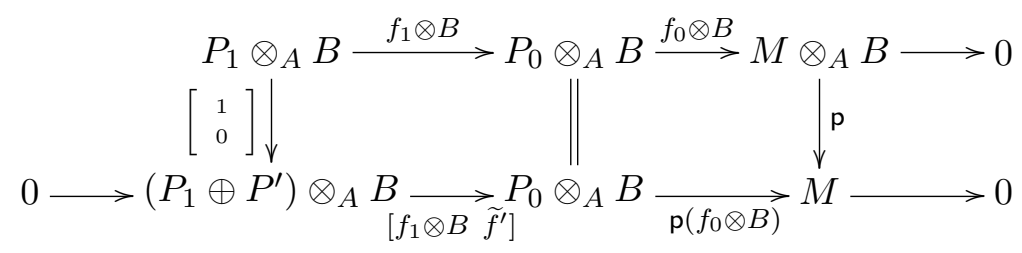

onde $\widetilde{f}^{\prime}$ é a restrição de $\widetilde{f}$ a $P^{\prime} \otimes_{A} B$.

Do epimorfismo $B$-linear $P_{1} \otimes_{A} B \oplus P \otimes_{A} B \longrightarrow \widetilde{P}_{1} \rightarrow 0$ segue que $\widetilde{P}_{1}$ é somando de $\left(P_{1} \oplus P\right) \otimes_{A} B$. Seja então $P^{\prime \prime}$ somando de $P_{1} \oplus P$ tal que $\widetilde{P}_{1}=P^{\prime \prime} \otimes_{A} B$, ou seja,

$$
0 \longrightarrow P^{\prime \prime} \otimes_{A} B \longrightarrow P_{0} \otimes_{A} B \underset{\mathrm{p}\left(f_{0} \otimes B\right)}{\longrightarrow} M \longrightarrow 0
$$


é uma resolução projetiva minimal de $M_{B}$. Aplicando o funtor $-\otimes_{B} A$ temos

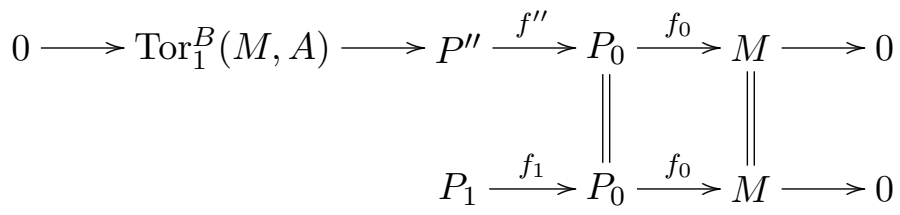

pois $\operatorname{Tor}_{1}^{B}\left(P_{0} \otimes_{A} B, A\right)=0$ uma vez que $P_{0} \otimes_{A} B$ é projetivo, e $M \otimes_{B} A \cong M \otimes_{B} \frac{B}{Q} \cong \frac{M}{M Q} \cong M_{A}$ uma vez que $M$ é anulado por $Q$.

De $f_{1}: P_{1} \longrightarrow$ Nuc $f_{0}$ ser cobertura projetiva e $f^{\prime \prime}: P^{\prime \prime} \longrightarrow$ Nuc $f_{0}$ ser um epimorfismo temos, pelo Lema 4.5, um epimorfismo $P^{\prime \prime} \longrightarrow P_{1} \rightarrow 0$ e portanto $P^{\prime \prime}=P_{1} \oplus P^{\prime}$ para algum $P^{\prime}$ submódulo de $P$, como queríamos.

Neste caso, $f_{1} \otimes B=\left[\begin{array}{ll}f_{1} \otimes B & \tilde{f}^{\prime}\end{array}\right]\left[\begin{array}{l}1 \\ 0\end{array}\right]$ é composição de monomorfismos e portanto um monomorfismo. Finalmente,

$$
0 \longrightarrow P_{1} \otimes_{A} B \stackrel{f_{1} \otimes B}{\longrightarrow} P_{0} \otimes_{A} B \stackrel{f_{0} \otimes B}{\longrightarrow} M \otimes_{A} B \longrightarrow 0
$$

é uma resolução projetiva minimal para $\left(M \otimes_{A} B\right)_{B}$, isto é, dp $\left(M \otimes_{A} B\right)_{B} \leq 1$.

Mostraremos agora que se um $A$-módulo indecomponível é tal que dp $M_{B} \leq 1$, então dp $M_{A} \leq 1$. Um resultado análogo vale também para a dimensão injetiva. Para isso precisamos de alguns lemas:

Lema 4.16 Seja $M$ um módulo em ind A. Então

(a) $\tau_{B}\left(M \otimes_{A} B\right) \cong \operatorname{Hom}_{A}\left({ }_{B} B_{A}, \tau_{A} M\right)$

(b) $\tau_{B}^{-1} \operatorname{Hom}_{A}\left({ }_{B} B_{A}, M\right) \cong\left(\tau_{A}^{-1} M\right) \otimes_{A} B_{B}$

Prova. Lembremos inicialmente que quando $P_{A}$ é projetivo, então valem os seguintes isomorfismos funtoriais:

$$
\begin{gathered}
B \otimes_{A} \operatorname{Hom}_{A}(P, A) \approx \operatorname{Hom}_{A}\left(P, B \otimes_{A} A\right) \approx \operatorname{Hom}_{A}\left(P, B_{A}\right) \\
\approx \operatorname{Hom}_{A}\left(P, \operatorname{Hom}_{B}(B, B)\right) \approx \operatorname{Hom}_{B}\left(P \otimes_{A} B, B\right) .
\end{gathered}
$$


(a) Seja $P_{1} \stackrel{f_{1}}{\longrightarrow} P_{0} \stackrel{f_{0}}{\longrightarrow} M \rightarrow 0$ uma apresentação projetiva minimal de $M_{A}$. Pelo Corolário 4.12,

$$
P_{1} \otimes_{A} B \stackrel{f_{1} \otimes B}{\longrightarrow} P_{0} \otimes_{A} B \stackrel{f_{0} \otimes B}{\longrightarrow} M \otimes_{A} B \rightarrow 0
$$

é uma apresentação projetiva minimal em $\bmod B$. Aplicando o funtor $B$-dual, pelo Lema 4.7 temos que

$$
\left(P_{0} \otimes_{A} B\right)^{t} \longrightarrow\left(P_{1} \otimes_{A} B\right)^{t} \longrightarrow \operatorname{Tr}^{B}\left(M \otimes_{A} B\right) \rightarrow 0
$$

é uma apresentação projetiva minimal em $\bmod B^{o p}$.

Por outro lado, aplicando o funtor $A$-dual na apresentação projetiva minimal de $M_{A}$, temos que $P_{0}^{t} \longrightarrow P_{1}^{t} \longrightarrow \operatorname{Tr}^{A} M \rightarrow 0$ é uma apresentação projetiva minimal em $\bmod A^{o p}$ e portanto

$$
B \otimes_{A} P_{0}^{t} \longrightarrow B \otimes_{A} P_{1}^{t} \longrightarrow B \otimes_{A} \operatorname{Tr}^{A} M \rightarrow 0
$$

também é uma apresentação projetiva minimal em mod $B^{o p}$.

Pelos isomorfismos iniciais, temos que $B \otimes_{A} P_{0}^{t}=B \otimes_{A} \operatorname{Hom}_{A}\left(P_{0}, A\right) \cong \operatorname{Hom}_{B}\left(P_{0} \otimes_{A} B, B\right)=$ $\left(P_{0} \otimes_{A} B\right)^{t}$ e $B \otimes_{A} P_{1}^{t} \cong\left(P_{1} \otimes_{A} B\right)^{t}$ e assim chegamos ao seguinte diagrama comutativo com linhas exatas:

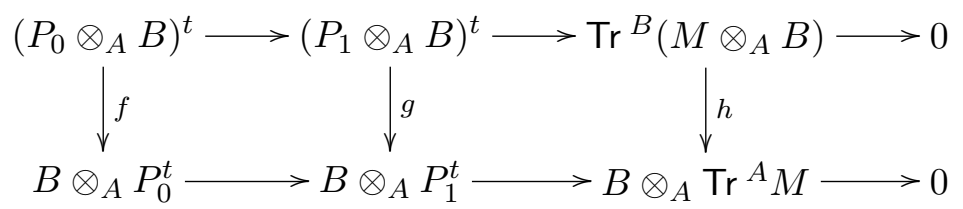

onde $f$ e $g$ são isomorfismos e $h$ é o morfismo induzido, o que implica que $h$ também é isomorfismo.

Finalmente,

$$
\begin{gathered}
\tau_{B}\left(M \otimes_{A} B\right)=D \operatorname{Tr}^{B}\left(M \otimes_{A} B\right) \cong D\left(B \otimes_{A} \operatorname{Tr}^{A} M\right)=H o m_{R}\left(B \otimes_{A} \operatorname{Tr}^{A} M, R\right) \cong \\
H \operatorname{Hom}_{A}\left(B_{A}, H o m_{R}\left(\operatorname{Tr}^{A} M, R\right)\right)=H o m_{A}\left(B, D \operatorname{Tr}^{A} M\right)=H o m_{A}\left(B, \tau_{A} M\right) .
\end{gathered}
$$

(b) Se $M$ é injetivo então $\operatorname{Hom}_{A}\left({ }_{B} B_{A}, M\right)$ também é injetivo e portanto

$$
\tau_{A}^{-1} M=0=\tau_{B}^{-1} \operatorname{Hom}_{A}\left({ }_{B} B_{A}, M\right)
$$


Agora se $M$ é não injetivo, então $M_{A} \cong \tau_{A} \tau_{A}^{-1} M$ e assim utilizando o item (a) para $\tau_{A}^{-1} M$ temos

$\tau_{B}^{-1} \operatorname{Hom}_{A}\left({ }_{B} B_{A}, M\right) \cong \tau_{B}^{-1} \operatorname{Hom}_{A}\left({ }_{B} B_{A}, \tau_{A}\left(\tau_{A}^{-1} M\right)\right) \cong \tau_{B}^{-1} \tau_{B}\left(\left(\tau_{A}^{-1} M\right) \otimes_{A} B_{B}\right) \cong\left(\tau_{A}^{-1} M\right) \otimes_{A} B_{B}$

Lema 4.17 Seja $M$ um módulo em ind A. Então

(a) $\mathrm{dp} M \otimes_{A} B_{B} \leq 1$ se, e somente se, dp $M_{A} \leq 1$ e $\operatorname{Hom}_{A}\left(D_{A} Q, \tau_{A} M\right)=0$.

(b) $\operatorname{di~Hom}_{A}\left({ }_{B} B_{A}, M\right)_{B} \leq 1$ se, e somente se, di $M_{A} \leq 1$ e $\operatorname{Hom}_{A}\left(\tau_{A}^{-1} M,{ }_{A} Q\right)=0$.

Prova.

(a) Pela Proposição 4.9, dp $\left(M \otimes_{A} B_{B}\right) \leq 1$ se, e somente se, $\operatorname{Hom}_{B}\left(D B, \tau_{B}\left(M \otimes_{A} B\right)\right)=0$. Observemos que $D B \otimes_{B} B_{A} \cong(D B)_{A} \cong D_{A} B \cong D_{A}(A \oplus Q) \cong D_{A} A \oplus D_{A} Q$. Daí, utilizando o lema anterior e o Teorema da Adjunção (1.7) chegamos aos seguintes isomorfismos:

$$
\begin{gathered}
\operatorname{Hom}_{B}\left(D B, \tau_{B}\left(M \otimes_{A} B\right) \cong \operatorname{Hom}_{B}\left(D B, \operatorname{Hom}_{A}\left({ }_{B} B_{A}, \tau_{A} M\right)\right) \cong \operatorname{Hom}_{A}\left(D B \otimes_{B} B_{A}, \tau_{A} M\right) \cong\right. \\
\operatorname{Hom}_{A}\left(D_{A} A \oplus D_{A} Q, \tau_{A} M\right) \cong \operatorname{Hom}_{A}\left(D_{A} A, \tau_{A} M\right) \oplus \operatorname{Hom}_{A}\left(D_{A} Q, \tau_{A} M\right) .
\end{gathered}
$$

Portanto dp $\left(M \otimes_{A} B_{B}\right) \leq 1 \Leftrightarrow \operatorname{Hom}_{A}\left(D_{A} A, \tau_{A} M\right)=0$ e $\operatorname{Hom}_{A}\left(D_{A} Q, \tau_{A} M\right)=0$, ou seja,

$$
\mathrm{dp}\left(M \otimes_{A} B_{B}\right) \leq 1 \Leftrightarrow \mathrm{dp} M_{A} \leq 1 \text { e } \operatorname{Hom}_{A}\left(D_{A} Q, \tau_{A} M\right)=0 .
$$

(b) Pela Proposição 4.9, di $\operatorname{Hom}_{A}\left({ }_{B} B_{A}, M\right)_{B} \leq 1 \Longleftrightarrow \operatorname{Hom}_{B}\left(\tau_{B}^{-1} \operatorname{Hom}_{A}\left({ }_{B} B_{A}, M\right), B\right)=0$.

Temos, nesse caso, os seguintes isomorfismos

$$
\begin{aligned}
& \operatorname{Hom}_{B}\left(\tau_{B}^{-1} \operatorname{Hom}_{A}\left({ }_{A} B_{B}, M\right), B\right) \cong \operatorname{Hom}_{B}\left(\left(\tau_{A}^{-1} M\right) \otimes_{A} B_{B}, B\right) \cong \operatorname{Hom}_{A}\left(\tau_{A}^{-1} M, B \otimes_{B} B\right) \cong \\
& \operatorname{Hom}_{A}\left(\tau_{A}^{-1} M,{ }_{A} B\right) \cong \operatorname{Hom}_{A}\left(\tau_{A}^{-1} M,{ }_{A} A \oplus_{A} Q\right) \cong \operatorname{Hom}_{A}\left(\tau_{A}^{-1} M,{ }_{A} A\right) \oplus \operatorname{Hom}_{A}\left(\tau_{A}^{-1} M,{ }_{A} Q\right)
\end{aligned}
$$

e portanto di $\operatorname{Hom}_{A}\left({ }_{B} B_{A}, M\right)_{B} \leq 1 \Leftrightarrow \operatorname{Hom}_{A}\left(\tau_{A}^{-1} M,{ }_{A} A\right)=0$ e $\operatorname{Hom}_{A}\left(\tau_{A}^{-1} M,{ }_{A} Q\right)=0$, ou seja,

$$
\operatorname{di} \operatorname{Hom}_{A}\left({ }_{B} B A, M\right)_{B} \leq 1 \Leftrightarrow \operatorname{di} M_{A} \leq 1 \mathrm{e} \operatorname{Hom}_{A}\left(\tau_{A}^{-1} M,{ }_{A} Q\right)=0 .
$$


Lema 4.18 Sejam A uma R-álgebra e $M$ um A-módulo em $\bmod A$. Então, $D M_{B} \cong D M_{A}$ em $\bmod B^{o p}$.

Prova. Lembremos que $M Q=0$ e portanto $M_{A} \cong M \otimes_{B} A_{A} \cong M \otimes_{B}(B / Q)_{A}$. Definiremos um isomorfismo $\Phi: D M_{B} \rightarrow D\left(M \otimes_{B}(B / Q)_{A}\right)$ :

Seja $f: M_{B} \rightarrow R, R$-linear. Definimos $g: M \times(B / Q) \rightarrow R$ por $g(m, b+Q):=f(m b)$ que é bilinear, logo existe uma $R$-linear $g^{\prime}: M \otimes(B / Q) \rightarrow R$ tal que $g^{\prime}(m \otimes(b+Q))=f(m b)$. Definimos $\Phi(f):=g^{\prime}$, ou seja, $\Phi(f)(m \otimes(b+Q))=f(m b)$. É facil ver que $\Phi$ é $R$-linear, vejamos que é também $B$-linear: dado $f \in D M_{B}$ e $b \in B$, então, $\forall m \in M$ e $\forall b^{\prime} \in B$ temos

$\Phi(b f)\left(m \otimes\left(b^{\prime}+Q\right)=(b f)\left(m b^{\prime}\right)=f\left(\left(m b^{\prime}\right) b\right)=f\left(m\left(b^{\prime} b\right)\right) \mathrm{e}\right.$

$(b \Phi(f))\left(m \otimes\left(b^{\prime}+Q\right)\right)=\Phi(f)\left(\left(m \otimes\left(b^{\prime}+Q\right)\right) b\right)=\Phi(f)\left(m \otimes\left(b^{\prime} b+Q\right)\right)=f\left(m\left(b^{\prime} b\right)\right)$.

Definiremos agora $\Psi: D\left(M \otimes_{B}(B / Q)_{A}\right) \rightarrow D M_{B}$. Seja $g: M \otimes_{B}(B / Q)_{A} \rightarrow R, R$-linear. Definimos $\Psi(g)(m):=g\left(m \otimes\left(1_{B}+Q\right)\right)$. Não é dificil ver que $\Psi$ é $B$-linear. Mostraremos que é a inversa de $\Phi$. Seja $g$ em $D\left(M \otimes_{B}(B / Q)_{A}\right)$, então, $\forall m \in M$ e $\forall r \in B$ temos

$$
\Phi(\Psi(g))(m \otimes(b+Q))=\Psi(g)(m b)=g\left(m b \otimes\left(1_{B}+Q\right)\right)=g(m \otimes(b+Q))
$$

e se $f \in D M_{B}$, então, $\forall m \in M$

$$
\Psi(\Phi(f))(m)=\Phi(f)\left(m \otimes\left(1_{B}+Q\right)=f\left(m 1_{B}\right)=f(m)\right.
$$

Proposição 4.19 Seja $M$ um módulo em ind $A$,

(a) se dp $M_{B} \leq 1$ então dp $M_{A} \leq 1$.

(b) se di $M_{B} \leq 1$ então di $M_{A} \leq 1$.

Prova.

(a) Como dp $M_{B} \leq 1$, pela Proposição 4.15 temos que dp $\left(M \otimes_{A} B_{B}\right) \leq 1$ e pelo lema anterior segue que dp $M_{A} \leq 1$. 
(b) Do isomorfismo do lema anterior, segue que $\mathrm{dp} D M_{B}=\mathrm{dp} D M_{A}$ como $B$-módulos. Pela hipótese, di $M_{B} \leq 1$ e então dp $D M_{B} \leq 1$. Portanto, dp $D M_{A} \leq 1$ como $B$-módulo. Pela parte (a), dp $D M_{A} \leq 1$ como $A$-módulo, isto é, di $M_{A} \leq 1$.

Para um $A$-módulo indecomponível, se dp $M_{B}=0$ então dp $M_{A}=0$ (Corolário 4.14) e se $\operatorname{dp} M_{B} \leq 1$ então dp $M_{A} \leq 1$ (Proposicao 4.19). Vejamos agora o que acontece quando dp $M_{B}=1$.

Exemplo 4.1 Sejam $k$ um corpo algebricamente fechado, B a álgebra dada pela aljava com a relação $\beta \alpha-\delta \gamma=0$ e $Q$ o ideal de $B$ gerado pelas classes de $\beta$ e $\gamma$. Então, pelo Teorema 3.8, a álgebra $B$ é uma extensão cindida por nilpotente de $A=\frac{B}{Q}$. Temos também que $A$ é dada pela aljava ${ }_{1}^{\alpha} \swarrow_{3}^{2} \nvdash_{\delta}^{4}$. O A-módulo simples $S_{3}$ associado ao vértice 3 é A-projetivo mas não é B-projetivo $e \mathrm{dp}\left(S_{3}\right)_{B}=1$, pois $0 \rightarrow P_{1}^{B} \rightarrow P_{3}^{B} \rightarrow S_{3} \rightarrow 0$ é uma resolução projetiva minimal em mod $B$.

\subsection{1 Álgebra hereditária e álgebra shod.}

Agora, a partir desse último resultado, podemos fazer afirmações a respeito das álgebras envolvidas. Vamos, então, lembrar as definições de álgebra hereditária e de álgebra shod.

Definição 4.8 Uma álgebra de Artin $C$ é dita hereditária se dim. gl. $C \leq 1$.

Definição 4.9 Uma álgebra de Artin $C$ é dita shod se para cada módulo indecomponível $M$ tem-se dp $M_{C} \leq 1$ ou di $M_{C} \leq 1$.

A partir das definições acima fica claro que toda álgebra hereditária é shod.

Teorema 4.20 Seja $B$ uma extensão cindida por nilpotente de $A$. Se B é uma álgebra hereditária então A também é uma álgebra hereditária.

Prova. Seja $M$ um $A$-módulo em ind $A$. Como $B$ é hereditária, então dp $M_{B} \leq 1$ e pela Proposição 4.19 temos dp $M_{A} \leq 1$. Logo, $\sup \{\operatorname{dp} M \mid M \in$ ind $A\} \leq 1$, isto é, dim. gl. $A \leq 1$. 
Teorema 4.21 Seja B uma extensão cindida por nilpotente de A. Se B é uma álgebra shod então A também é uma álgebra shod.

Prova. Seja $M$ um $A$-módulo indecomponível em $\bmod A$. Como $B$ é shod então dp $M_{B} \leq 1$ ou di $M_{B} \leq 1$ e pela Proposição 4.19 temos dp $M_{A} \leq 1$ ou di $M_{A} \leq 1$. Logo, $A$ é shod.

A pergunta natural é o que podemos falar sobre a recíproca desses teoremas. Como mostram os exemplos a seguir, não são verdadeiras em geral.

Exemplo 4.2 Sejam $A$ e $B$ as k-álgebras do Exemplo 4.1. Então

$$
0 \rightarrow P_{1} \rightarrow P_{2} \oplus P_{3} \rightarrow P_{4} \rightarrow S_{4} \rightarrow 0
$$

é uma resolução projetiva minimal para o $B$-módulo simples $S_{4}$ e, portanto, $\mathrm{dp} S_{4}=2>1$, ou seja, $B$ não é hereditária. No entanto, em $A$ temos que $\mathrm{dp} S_{1}=\mathrm{dp} S_{3}=0$ e $\mathrm{dp} S_{2}=\mathrm{dp} S_{4}=1$, isto é, dim. gl. $A=1$.

Exemplo 4.3 Seja $k$ um corpo algebricamente fechado. Consideremos B a k-álgebra dada pela

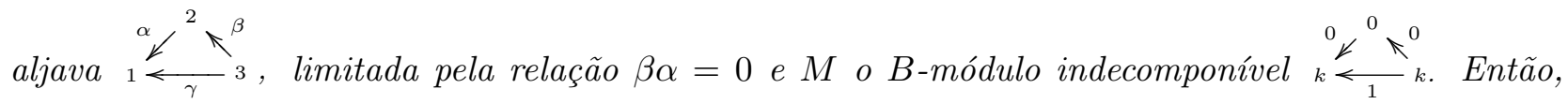
$0 \rightarrow P_{1} \rightarrow P_{2} \rightarrow P_{3} \rightarrow M \rightarrow 0$ é uma resolução projetiva minimal para $M$ e $0 \rightarrow M \rightarrow I_{1} \rightarrow$ $I_{2} \rightarrow I_{3} \rightarrow 0$ é uma resolução injetiva minimal para $M$, ou seja, dp $M=2$ e di $M=2$. Portanto, $B$ não é shod.

Sejam $Q$ o ideal de $B$ gerado por $\beta$ e $A=\frac{B}{Q}$. Então, $B$ é uma extensão cindida de $A$ pelo ideal nilpotente $Q$. A álgebra $A$ é dada por $2 \stackrel{\alpha}{\longrightarrow} 1<{ }^{\gamma} 3$ que é hereditária e portanto shod. 


\section{Capítulo 5}

\section{Parte direita e parte esquerda}

No final do capítulo anterior vimos, quando $B$ é uma extensão cindida por nilpotente de $A$, que $A$ é uma álgebra hereditária (ou shod) sempre que $B$ for hereditária (ou shod). [Teorema 4.20 e Teorema 4.21]

Veremos nesse capítulo que isso também é verdade para outras classes de álgebras. As classes que aqui serão apresentadas podem ser caracterizadas pelas partes direita $\mathcal{R}$ e esquerda $\mathcal{L}$ de sua respectiva categoria de módulos.

Começamos, portanto, definindo as categorias $\mathcal{L}_{A}, \mathcal{L}_{B}, \mathcal{R}_{A}$ e $\mathcal{R}_{B}$ e relacionando-as.

\subsection{Parte direita e parte esquerda da categoria de módulos}

Aqui todas as álgebras são $R$-álgebras de Artin. As álgebra $A$ e $B$ são tais que $B$ é uma extensão cindida de $A$ pelo ideal nilpotente $Q$ e $C$ denotará uma álgebra qualquer.

Definição 5.1 Dados dois $C$-módulos indecomponíveis $M$ e $N$, um caminho de $M$ para $N$ de comprimento $t \geq 0$ em ind $C$ é uma sequência

$$
M=M_{0} \stackrel{f_{1}}{\longrightarrow} M_{1} \longrightarrow \cdots \longrightarrow M_{t-1} \stackrel{f_{t}}{\longrightarrow} M_{t}=N
$$

onde cada $M_{i} \in$ ind $C$ e cada $f_{i}$ é um morfismo não nulo.

Notação: $M \rightsquigarrow N$

Nesse caso dizemos que $M$ é um predecessor de $N$ e que $N$ é um sucessor de $M$. 
Notemos que um $C$-módulo $M$ é sempre sucessor e predecessor dele mesmo, basta considerar um caminho de comprimento $t=0$.

\section{Definição 5.2}

$A$ parte esquerda de $\bmod C$, denotada por $\mathcal{L}_{C}$, é a subcategoria plena de ind $C$ cujos objetos são os módulos para os quais a dimensão projetiva de seus predecessores é menor ou igual a 1. Também escreveremos $\mathcal{L}_{C}=\{X \in$ ind $C \mid$ se $Y \rightsquigarrow X$ então dp $Y \leq 1\}$.

$A$ parte direita de $\bmod C$, denotada por $\mathcal{R}_{C}$, é a subcategoria plena de ind $C$ cujos objetos são os módulos para os quais a dimensão injetiva de seus sucessores é menor ou igual a 1. Também escreveremos $\mathcal{R}_{C}=\{X \in$ ind $C \mid$ se $X \rightsquigarrow Y$ então di $Y \leq 1\}$.

Não é difícil ver que, com as definições acima, a subcategoria $\mathcal{L}_{C}$ é fechada para predecessores e a subcategoria $\mathcal{R}_{C}$ é fechada para sucessores.

Consideremos agora o caso em que $B$ é uma extensão cindida por nilpotente de $A$ e comparemos as partes direita e esquerda de $\bmod A$ e $\bmod B$.

Lema 5.1 Sejam B uma extensão cindida de $A$ pelo ideal nilpotente $Q$ e $M_{A}$ um A-módulo indecomponível.

1. Se $M \otimes_{A} B \in \mathcal{L}_{B}$ então $M \in \mathcal{L}_{A}$.

2. $S e \operatorname{Hom}_{A}(B, M) \in \mathcal{R}_{B}$ então $M \in \mathcal{R}_{A}$.

3. Se $M \otimes_{A} B \in \mathcal{R}_{B}$ então $M \in \mathcal{R}_{A}$.

4. $\operatorname{Se} \operatorname{Hom}_{A}(B, M) \in \mathcal{L}_{B}$ então $M \in \mathcal{L}_{A}$.

Prova. Observemos inicialmente que se $L \in$ ind $A$ então, pelo Lema 2.6, $L \otimes_{A} B \in$ ind $B$ e $\operatorname{Hom}_{A}(B, L) \in$ ind $B$. Além disso, se $f: L \rightarrow \hat{L}$ é não nulo segue que $f \otimes B: L \otimes_{A} B \rightarrow \hat{L} \otimes_{A} B$ e ${ }_{A}(B, f):=\operatorname{Hom}_{A}(B, f): \operatorname{Hom}_{A}(B, L) \rightarrow \operatorname{Hom}_{A}(B, \hat{L})$ são não nulos, pois $f \otimes B \otimes A \cong f \cong$ $\operatorname{Hom}_{B}\left(A, \operatorname{Hom}_{A}(B, f)\right)$. 
1. Seja $L \in$ ind $A$ um predecessor de $M$ e consideremos $L=L_{0} \stackrel{f_{1}}{\rightarrow} L_{1} \rightarrow \cdots \rightarrow L_{n-1} \stackrel{f_{n}}{\rightarrow} L_{n}=M$ um caminho de $L$ para $M$ em ind $A$. Aplicando o funtor $-\otimes_{A} B$, temos que

$$
L \otimes_{A} B=L_{0} \otimes_{A} B \stackrel{f_{1} \otimes B}{\longrightarrow} L_{1} \otimes_{A} B \longrightarrow \cdots \longrightarrow L_{n-1} \otimes_{A} B \stackrel{f_{n} \otimes B}{\longrightarrow} L_{n} \otimes_{A} B=M \otimes_{A} B
$$

é um caminho de $L \otimes_{A} B$ para $M \otimes_{A} B$ em ind $B$. Como $M \otimes_{A} B \in \mathcal{L}_{B}$ então dp $L \otimes_{A} B \leq 1$ e pelo Lema 4.17 segue que dp $L_{A} \leq 1$ e portanto $M \in \mathcal{L}_{A}$.

2. Seja $L \in$ ind $A$ um sucessor de $M$ e consideremos $M=L_{0} \stackrel{f_{1}}{\rightarrow} L_{1} \rightarrow \cdots \rightarrow L_{n-1} \stackrel{f_{n}}{\rightarrow} L_{n}=L$ um caminho de $M$ para $L$ em ind $A$. Aplicando o funtor $\operatorname{Hom}_{A}(B,-)$, temos que

$$
\operatorname{Hom}_{A}(B, M) \stackrel{A\left(B, f_{1}\right)}{\longrightarrow} \operatorname{Hom}_{A}\left(B, L_{1}\right) \longrightarrow \cdots \longrightarrow \operatorname{Hom}_{A}\left(B, L_{n-1}\right) \stackrel{A\left(B, f_{n}\right)}{\longrightarrow} \operatorname{Hom}_{A}(B, L)
$$

é um caminho de $\operatorname{Hom}_{A}(B, M)$ para $\operatorname{Hom}_{A}(B, L)$ em ind $B$. Como $\operatorname{Hom}_{A}(B, M) \in \mathcal{R}_{B}$ então $\operatorname{di~}_{\operatorname{Hom}}(B, L) \leq 1$ e pelo Lema 4.17 segue que di $L_{A} \leq 1$ e portanto $M \in \mathcal{R}_{A}$.

3. Utilizando o Teorema da Adjunção (1.7), o isomorfismo do Exemplo 1.9 e lembrando que $B_{A} \cong A_{A} \oplus Q_{A}$, temos os seguintes isomorfismos de $R$-módulos:

$$
\begin{aligned}
\operatorname{Hom}_{B}\left(M \otimes_{A} B, \operatorname{Hom}_{A}(B B, M)\right) & \cong \operatorname{Hom}_{A}\left(M \otimes_{A} B \otimes_{B} B_{A}, M\right) \\
& \cong \operatorname{Hom}_{A}\left(M \otimes_{A} B, M\right) \\
& \cong \operatorname{Hom}_{A}\left(M \otimes_{A}\left(A_{A} \oplus Q_{A}\right), M\right) \\
& \cong \operatorname{Hom}_{A}\left(\left(M \otimes_{A} A_{A}\right) \oplus\left(M \otimes_{A} Q_{A}\right), M\right) \\
& \cong \operatorname{Hom}_{A}\left(M_{A} \oplus\left(M \otimes_{A} Q\right), M\right) \\
& \cong \operatorname{Hom}_{A}(M, M) \oplus \operatorname{Hom}_{A}\left(M \otimes_{A} Q, M\right)
\end{aligned}
$$

Como $\operatorname{Hom}_{A}(M, M)$ é não nulo, então existe um $B$-homomorfismo não nulo de $M \otimes_{A} B$ para $\operatorname{Hom}_{A}(B, M)$, ou seja $\operatorname{Hom}_{A}(B, M)$ é um sucessor de $M \otimes_{A} B$. Como $M \otimes_{A} B \in \mathcal{R}_{B}$ e $\mathcal{R}_{B}$ é fechado para sucessores então $\operatorname{Hom}_{A}(B, M) \in \mathcal{R}_{B}$. Pelo item 2, temos que $M \in \mathcal{R}_{A}$.

4. Pelo isomorfismo apresentado em 3 temos que $M \otimes_{A} B$ é um predecessor de $\operatorname{Hom}_{A}(B, M)$. Como $\operatorname{Hom}_{A}(B, M) \in \mathcal{L}_{B}$ e $\mathcal{L}_{B}$ é fechado para predecessores então $M \otimes_{A} B \in \mathcal{L}_{B}$. Pelo item 1 temos $M \in \mathcal{L}_{A}$. 


\section{$5.2 \quad$ Álgebras determinadas por $\mathcal{L}$ e $\mathcal{R}$}

As classes de álgebra aqui tratadas serão: laura, colada à direita, colada à esquerda, fracamente shod e quase inclinada. As definições apresentadas não são necessariamente as originais, mas obviamente são equivalentes.

Precisamos primeiramente da definição de subcategorias finita e cofinita:

Definição 5.3 Uma subcategoria $\mathcal{D}$ de ind $C$ é dita finita em ind $C$ se contém apenas um número finito de $C$-módulos. E é dita cofinita em ind $C$ se a subcategoria ind $C \backslash \mathcal{D}$ é finita em ind $C$.

Definição 5.4 Uma álgebra de Artin $C$ é dita de tipo de representação finito se a categoria ind $C$ for finita. Caso contrário dizemos que $C$ é de tipo de representação infinito.

Como trataremos da finitude de subcategorias de ind $A$ e ind $B$ usaremos o seguinte lema:

Lema 5.2 Seja $M_{A}$ uma A-módulo. Então, $M_{A} \cong N_{A}$ se, e somente se, $M \otimes_{A} B \cong N \otimes_{A} B$.

Prova. Seja $f: M_{A} \rightarrow N_{A}$ um isomorfismo. Então $f \otimes B: M \otimes_{A} B \rightarrow N \otimes_{A} B$ também será um isomorfismo cuja inversa é $f^{-1} \otimes B: N \otimes_{A} B \rightarrow M \otimes_{A} B$, pois $(f \otimes B)\left(f^{-1} \otimes B\right)=f f^{-1} \otimes B=$ $i d_{N} \otimes B=i d_{N \otimes_{A} B}$ e $\left(f^{-1} \otimes B\right)(f \otimes B)=f^{-1} f \otimes B=i d_{M} \otimes B=i d_{M \otimes_{A} B}$.

Reciprocamente, se $g: M \otimes_{A} B \rightarrow N \otimes_{A} B$ então $g \otimes A: M \otimes_{A} B \otimes_{B} A \rightarrow N \otimes_{A} B \otimes_{B} A$ também será um isomorfismo e portanto $M_{A} \cong M \otimes_{A} B \otimes_{B} A \cong N \otimes_{A} B \otimes_{B} A \cong N_{A}$.

Corolário 5.3 Se B é de tipo de representação finito, então A também é de tipo de representação finito.

Prova. Suponhamos que ind $A$ é infinita, pelo lema anterior o conjunto $\left\{M \otimes_{A} B \mid M \in\right.$ ind $\left.A\right\} \subseteq$ ind $B$ é infinito. Contradição, pois ind $B$ é finita.

\subsection{1 Álgebra laura.}

Essa classe de álgebras foi introduzida em [3] e contém as demais classes já citadas: colada (à direita e à esquerda), fracamente shod, shod, quase inclinada e hereditária. (Ver também [5]) 
Definição 5.5 Uma álgebra de Artin $C$ é dita uma álgebra laura se a união $\mathcal{L}_{C} \cup \mathcal{R}_{C}$ for cofinita $e m$ ind $C$.

Teorema 5.4 Seja B uma extensão cindida por nilpotente de A. Se B é uma álgebra laura então A também é laura.

Prova. Seja $M \in$ ind $A$ tal que $M \notin \mathcal{L}_{A} \cup \mathcal{R}_{A}$. Se $M \otimes_{A} B \in \mathcal{L}_{B}$ então pelo item 1 do Lema 5.1 teríamos $M \in \mathcal{L}_{A}$. Da mesma forma, pelo item 3 do mesmo lema, temos que $M \otimes_{A} B \notin \mathcal{R}_{B}$ e portanto $M \otimes_{A} B \in$ ind $B$ mas $M \otimes_{A} B \notin \mathcal{L}_{B} \cup \mathcal{R}_{B}$.

Como $B$ é laura, então $\mathcal{L}_{B} \cup \mathcal{R}_{\mathcal{B}}$ é cofinita em ind $B$ e consequentemente $\mathcal{L}_{A} \cup \mathcal{R}_{A}$ é cofinita em ind $A$. Caso contrário, pelo Lema 5.2 teríamos que ind $B \backslash\left(\mathcal{L}_{B} \cup \mathcal{R}_{B}\right)$ seria infinita. Portanto, $A$ é laura.

\subsection{2 Álgebras coladas à direita e à esquerda.}

As álgebras coladas foram introduzidas em [2].

Definição 5.6 ( [2], 3.2) Seja C uma álgebra de Artin.

(a) $C$ é dita colada à esquerda $s e\{M \in$ ind $C \mid$ di $M \leq 1\}$ é cofinita em ind $C$.

(b) $C$ é dita colada à direita $s e\{M \in$ ind $C \mid \mathrm{dp} M \leq 1\}$ é cofinita em ind $C$.

Em [3] mostra-se a seguinte equivalência:

Lema 5.5 ( [3], 2.2) Seja $C$ uma álgebra de Artin.

(a) $C$ é colada à esquerda se, e somente se, $\mathcal{R}_{C}$ é cofinita em ind $C$.

(b) $C$ é colada à direita se, e somente se, $\mathcal{L}_{C}$ é cofinita em ind $C$.

Teorema 5.6 Seja B uma extensão cindida por nilpotente de A.

(a) Se B é uma álgebra colada à direita então A também é colada à direita.

(b) Se B é uma álgebra colada à esquerda então A também é colada à esquerda. 
Prova.

(a) Suponhamos que $B$ seja colada à direita. Seja $M \in$ ind $A$ tal que $M \notin \mathcal{R}_{A}$. Pelo item 3 do Lema 5.1 temos que $M \otimes_{A} B \notin \mathcal{R}_{B}$. Pelo lema anterior $\mathcal{R}_{B}$ é cofinita em ind $B$, daí segue do Lema 5.2 que $\mathcal{R}_{A}$ é cofinita em ind $A$. Portanto, $A$ é colada à direita.

(b) Analogamente, se $M \in$ ind $A$ é tal que $M \notin \mathcal{L}_{A}$, pelo item 1 do Lema $5.1 M \otimes_{A} B \notin \mathcal{L}_{B}$. Daí se $B$ é colada à esquerda temos que $\mathcal{L}_{B}$ é cofinita em ind $B$ e o Lema 5.2 implica que $\mathcal{L}_{A}$ é cofinita em ind $A$, isto é, $A$ é colada à esquerda.

\subsection{3 Álgebra fracamente shod.}

Está é uma subclasse da classe de álgebras laura e como o nome sugere contém a classe das álgebras shod.

Definição 5.7 ( [15], 2.3) Uma álgebra de Artin $C$ é chamada fracamente shod se existe um inteiro positivo $n$ tal que todo caminho em ind $C$ de um injetivo para um projetivo tem comprimento no máximo $n$.

Mostra-se em [4] que basta olhar os caminhos de módulos em ind $C \backslash \mathcal{L}_{C}$ para módulos em ind $C \backslash \mathcal{R}_{C}$ :

Lema 5.7 ( $[4], 1.1)$ Uma álgebra de Artin $C$ é fracamente shod se existe um inteiro positivo $n$ tal que todo caminho em ind $C$ de um módulo que não está em $\mathcal{L}_{C}$ para um módulo que não está em $\mathcal{R}_{C}$ tem comprimento no máximo n.

Teorema 5.8 Seja B uma extensão cindida por nilpotente de A. Se B é uma álgebra fracamente shod então A também é fracamente shod.

Prova. Sejam $M, N \in$ ind $A$ com $M \notin \mathcal{L}_{A}$ e $N \notin \mathcal{R}_{A}$ tais que $M$ é predecessor de $N$. Consideremos $M=L_{0} \stackrel{f_{1}}{\rightarrow} L_{1} \rightarrow \cdots \rightarrow L_{n-1} \stackrel{f_{n}}{\rightarrow} L_{n}=N$ um caminho de $M$ para $N$ em ind $A$. Aplicando o funtor $-\otimes_{A} B$, temos que

$$
M \otimes_{A} B=L_{0} \otimes_{A} B \stackrel{f_{1} \otimes B}{\longrightarrow} L_{1} \otimes_{A} B \longrightarrow \cdots \longrightarrow L_{n-1} \otimes_{A} B \stackrel{f_{n} \otimes B}{\longrightarrow} L_{n} \otimes_{A} B=N \otimes_{A} B
$$


é um caminho de $M \otimes_{A} B$ para $N \otimes_{A} B$ de comprimento $n$ em ind $B$. Pelo Lema 5.1, $M \otimes_{A} B \notin \mathcal{L}_{B}$ e $N \otimes_{A} B \notin \mathcal{R}_{B}$. Como $B$ é fracamente shod, pelo lema anterior, existe $n_{0}$ tal que $n \leq n_{0}$. Portanto, $A$ é fracamente shod.

\subsection{4 Álgebra shod}

O resultado para álgebras shod já foi enunciado em 4.21. Incluímos aqui uma definição equivalente à dada anteriormente, envolvendo as partes direita e esquerda da categoria de módulos, que será utilizada nos exemplos. Há também uma nova versão para a demonstração do Teorema 4.21.

Essa classe de álgebras foi introduzida em [14] onde também encontramos a equivalência:

Proposição 5.9 ( [14], 2.1) Seja C uma álgebra de Artin.

Então $C$ é uma álgebra shod se, e somente se, ind $C=\mathcal{L}_{C} \cup \mathcal{R}_{C}$.

Prova 2.[ para o Teorema 4.21] Suponhamos que $B$ é shod e seja $M_{A} \in$ ind $A$. Como $M \otimes_{A} B \in$ ind $B$ então, pela proposição anterior, $M \otimes_{A} B \in \mathcal{L}_{B}$ ou $\mathcal{R}_{B}$. Pelos itens 1 e 3 do Lema 5.1 teremos que $M \in \mathcal{L}_{A}$ ou $M \in \mathcal{R}_{A}$, ou seja ind $A \subseteq \mathcal{L}_{A} \cup \mathcal{R}_{A}$. Como a outra inclusão é óbvia, segue que $A$ é shod.

\subsection{5 Álgebra quase inclinada.}

Pela definição abaixo, é fácil ver que a classe das álgebras inclinadas é uma subclasse das álgebras shod.

Definição 5.8 ( [16], II) Uma álgebra de Artin $C$ é dita quase inclinada se for shod e dim. gl. $C \leq 2$.

Mostra-se em [16] que vale:

Lema 5.10 ( [16], II 1.14) Seja C uma álgebra de Artin. Então $C$ é quase inclinada se, e somente se, $\mathcal{L}_{C}$ contém todos os módulos projetivos de ind $C$.

Teorema 5.11 Seja B uma extensão cindida por nilpotente de A. Se B é uma álgebra quase inclinada então A também é quase inclinada. 
Prova. Seja $P \in$ ind $A$ um módulo projetivo, mostraremos que $P \in \mathcal{L}_{A}$. Pelo Lema 2.6 temos que $P \otimes_{A} B$ é projetivo e indecomponível e, como $B$ é quase inclinada, então $P \otimes_{A} B \in \mathcal{L}_{B}$. Pelo item 1 do Lema 5.1 segue que $P \in \mathcal{L}_{A}$.

\subsection{6 Álgebra disfarçada.}

Esta é uma classe de álgebras de tipo de representação infinito, que pertence às demais classes já citadas nesse capítulo. Sua definição pode ser encontrada, por exemplo em [8]. Sua caracterização pode ser feita da seguinte forma:

Lema 5.12 ( [2], 3.4) Seja $C$ uma álgebra de Artin de tipo de representação infinito. Então, $C$ é uma álgebra disfarçada se, e somente se, $C$ é colada à esquerda e $C$ é colada à direita.

Gostaríamos de enunciar um resultado para essa classe, análogo aos aqui apresentados. Porém, se uma álgebra $B$, de tipo de representação infinito, é extensão cindida de uma álgebra $A$ por um ideal nilpotente, não podemos garantir que $A$ também seja de tipo infinito.

Exemplo 5.1 Sejam $k$ um corpo algebricamente fechado, $\Delta_{B}$ a aljava $1 \longleftarrow_{\beta}^{\alpha} 2$ e $\Delta_{A}$ a aljava $1 \stackrel{\alpha}{\longleftarrow} 2$. Então, $k \Delta_{B}$ é uma extensão cindida de $k \Delta_{A}$ pelo ideal gerado pela flecha $\beta$. A álgebra $B$ é de tipo de representação infinito, mas $k \Delta_{A}$ é de tipo finito.

Acrescentaremos, portanto, a hipótese de que $A$ seja de tipo de representação infinito, pois o Corolário 5.3 garante que $B$ também será de tipo infinito.

Teorema 5.13 Sejam A uma álgebra de tipo de representação infinito e $B$ uma extensão cindida por nilpotente de A. Se B é uma álgebra disfarçada, então A também é uma álgebra disfarçada.

Prova. Como $B$ é disfarçada, pelo Lema 5.12, $B$ é colada à esquerda e à direita. Pelo Teorema 5.6, segue que $A$ também é colada à esquerda e à direita e como $A$ é de tipo infinito, segue que $A$ é também disfarçada. 


\subsection{Exemplos}

Veremos que as recíprocas dos teoremas acima não são verdadeiras. Os exemplos serão dados no contexto de álgebras dadas por aljavas com relações. Por isso, consideremos um corpo $k$ algebricamente fechado.

Para decidir se a álgebra que estivermos estudando é de uma determinada classe olharemos a chamada Aljava de Auslander-Reiten da álgebra. O estudo de tal aljava é suficiente para obter as informações da categoria dos módulos indecomponíveis que precisamos. Esse fato não será detalhado neste trabalho, apenas faremos algumas considerações sobre a aljava de Auslander-Reiten de uma álgebra no Apêndice A.

Para decidir se uma álgebra é extensão cindida de outra usaremos a técnica do Teorema 3.8.

\section{$1^{\mathrm{o}}$ Exemplo.}

Seja $B$ a $k$-álgebra dada pela aljava todo $i, j$, e $\beta_{1} \gamma_{1}=0$, ou seja, $B=\frac{k \Delta}{\mathcal{I}}$, onde $\Delta$ é a aljava acima e $\mathcal{I}$ é o ideal gerado por $\alpha_{i} \beta_{j}, \gamma_{i} \delta_{j}$ e $\beta_{1} \gamma_{1}$.

Segue um esquema da aljava de Auslander-Reiten para $B$, onde destacamos $\mathcal{L}_{B}$ e $\mathcal{R}_{B}$ :

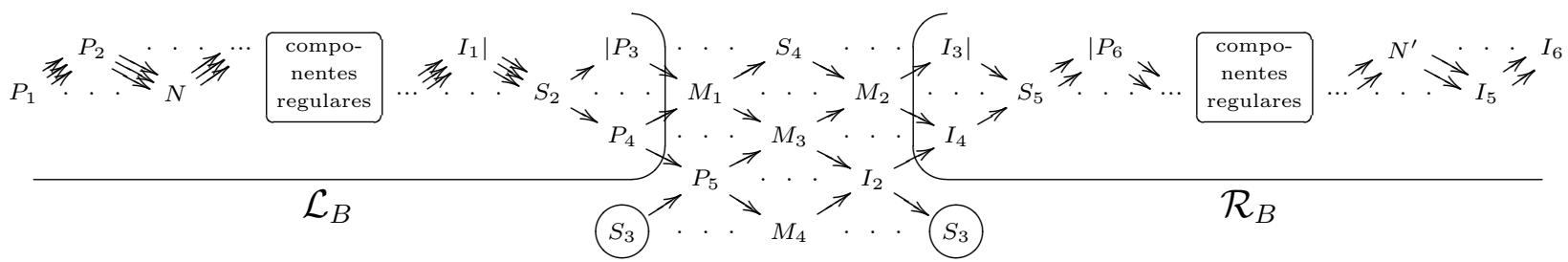

onde $S_{i}, P_{i}$ e $I_{i}$ são os módulos indecomponíveis simples, projetivo e injetivo referentes ao vértice $i$, respectivamente. Além disso, $N=83{ }_{0}^{0} 00, N^{\prime}=00{ }_{0}^{0}{ }^{23}, M_{1}=01{ }_{1}^{1} 00, M_{2}=00{ }_{1}^{1}{ }_{10}, M_{3}=01{ }_{1}^{2} 0$ e $M_{4}=01{ }_{1}^{0} 10$.

Vejamos a que classes de álgebras $B$ pertence:

- $B$ é laura, pois (ind $B) \backslash\left(\mathcal{L}_{B} \cup \mathcal{R}_{B}\right)=\left\{S_{3}, M_{1}, P_{5} . S_{4}, M_{3}, M_{4}, M_{2}, I_{2}\right\}$ é finita. 
- $B$ não é colada à esquerda, pois ind $B \backslash \mathcal{R}_{B}$ não é finita.

- $B$ não é colada à direita, pois ind $B \backslash \mathcal{L}_{B}$ não é finita.

- $B$ não é shod, pois $S_{3} \notin \mathcal{L}_{B} \cup \mathcal{R}_{B}$ por exemplo.

- $B$ não é quase inclinada pois não é shod.

- $B$ não é fracamente shod, pois não existe um inteiro que limite os caminhos de $I_{2}$ para $P_{5}$ da forma:

$$
I_{2} \rightarrow S_{3} \rightarrow P_{5} \rightarrow M_{3} \rightarrow I_{2} \rightarrow S_{3} \rightarrow P_{5} \rightarrow M_{3} \rightarrow I_{2} \rightarrow \ldots \rightarrow P_{5}
$$

Vejamos agora alguns exemplos de $k$-álgebras $A_{i}$ tais que $B$ seja uma extensão cindida por um ideal nilpotente $Q_{i}$ :

$A_{1}$ :

Consideremos $Q_{1}$ o ideal de $B$ gerado por $\delta_{3}$ e $A_{1}=B / Q_{1}$. Então, $B$ é uma extensão cindida de $A_{1}$ pelo nilpotente $Q_{1}$. Temos que $A_{1}$ é a álgebra dada pela aljava relações herdadas de $B$. Um esquema para a aljava de Auslander-Reiten dessa álgebra é:

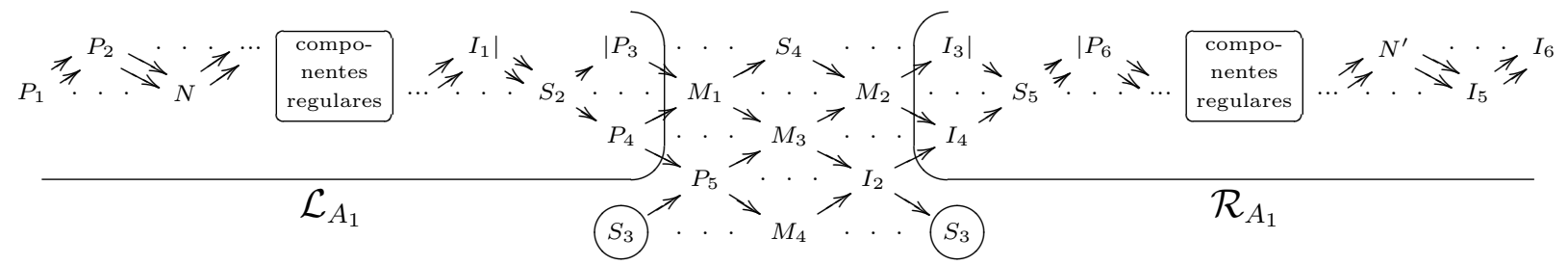

Temos então que:

- $A_{1}$ é laura, pois (ind $\left.A_{1}\right) \backslash\left(\mathcal{L}_{A_{1}} \cup \mathcal{R}_{A_{1}}\right)=\left\{S_{3}, M_{1}, P_{5} . S_{4}, M_{3}, M_{4}, M_{2}, I_{2}\right\}$ é finita.

- $A_{1}$ não é colada à esquerda, pois ind $A_{1} \backslash \mathcal{R}_{A_{1}}$ não é finita.

- $A_{1}$ não é colada à direita, pois ind $A_{1} \backslash \mathcal{L}_{A_{1}}$ não é finita. 
- $A_{1}$ não é shod, pois $S_{3} \notin \mathcal{L}_{A_{1}} \cup \mathcal{R}_{A_{1}}$ por exemplo.

- $A_{1}$ não é quase inclinada, pois não é shod.

- $A_{1}$ não é fracamente shod, pois não existe um inteiro que limite os caminhos de $I_{2}$ para $P_{5}$ da forma:

$$
I_{2} \rightarrow S_{3} \rightarrow P_{5} \rightarrow M_{3} \rightarrow I_{2} \rightarrow S_{3} \rightarrow P_{5} \rightarrow M_{4} \rightarrow I_{2} \rightarrow \ldots \rightarrow P_{5}
$$

$A_{2}$ :

Consideremos $Q_{2}$ o ideal de $B$ gerado por $\alpha_{1}$ e por $\alpha_{2}$. Então $B$ é uma extensão cindida de $A_{2}=B / Q_{2}$ pelo nilpotente $Q_{2}$. Além disso, $A_{2}$ é dada pela aljava relações herdadas de $B$. Um esquema para a aljava de Auslander-Reiten dessa álgebra é:

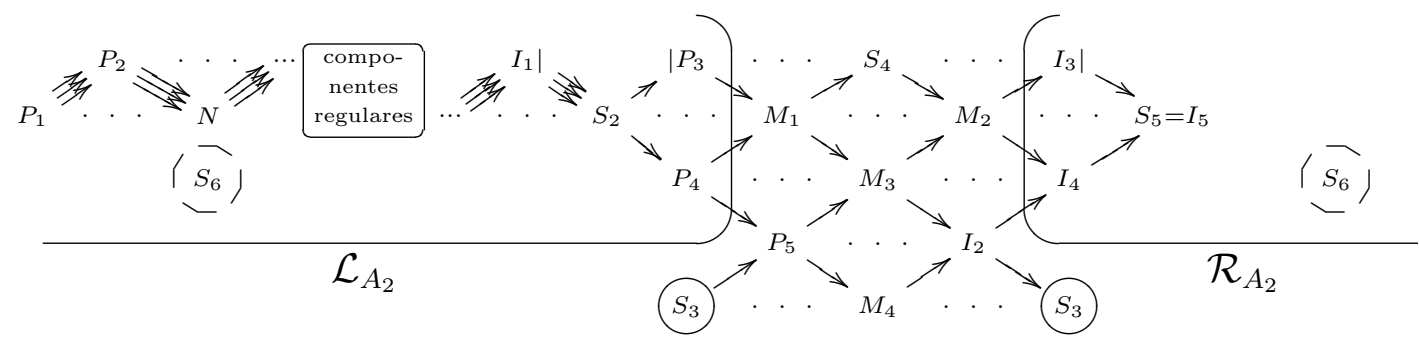

Então,

- $A_{2}$ é laura, pois (ind $\left.A_{2}\right) \backslash\left(\mathcal{L}_{A_{2}} \cup \mathcal{R}_{A_{2}}\right)=\left\{S_{3}, M_{1}, P_{5} . S_{4}, M_{3}, M_{4}, M_{2}, I_{2}\right\}$ é finita.

- $A_{2}$ não é colada à esquerda, pois ind $A_{2} \backslash \mathcal{R}_{A_{2}}$ não é finita.

- $A_{2}$ é colada à direita, pois ind $A_{2} \backslash \mathcal{L}_{A_{2}}=\left\{S_{3}, M_{1}, P_{5} . S_{4}, M_{3}, M_{4}, M_{2}, I_{2}, I_{3}, I_{4}, S_{5}\right\}$ é finita.

- $A_{2}$ não é shod, pois $S_{3} \notin \mathcal{L}_{A_{2}} \cup \mathcal{R}_{A_{2}}$ por exemplo.

- $A_{2}$ não é quase inclinada pois não é shod.

- $A_{2}$ não é fracamente shod, pois não existe um inteiro que limite os caminhos de $I_{2}$ para $P_{5}$ da 
forma:

$$
I_{2} \rightarrow S_{3} \rightarrow P_{5} \rightarrow M_{3} \rightarrow I_{2} \rightarrow S_{3} \rightarrow P_{5} \rightarrow M_{3} \rightarrow I_{2} \rightarrow \ldots \rightarrow P_{5}
$$

$A_{3}$ :

Consideremos $Q_{3}$ o ideal de $B$ gerado por $\beta_{2}$ e por $\gamma_{2}$. Temos que $B$ uma extensão cindida de $A_{3}=B / Q_{3}$ pelo nilpotente $Q_{3}$. Além disso, $A_{3}$ é dada pela aljava relações herdadas de $B$. Um esquema para a aljava de Auslander-Reiten dessa álgebra é:

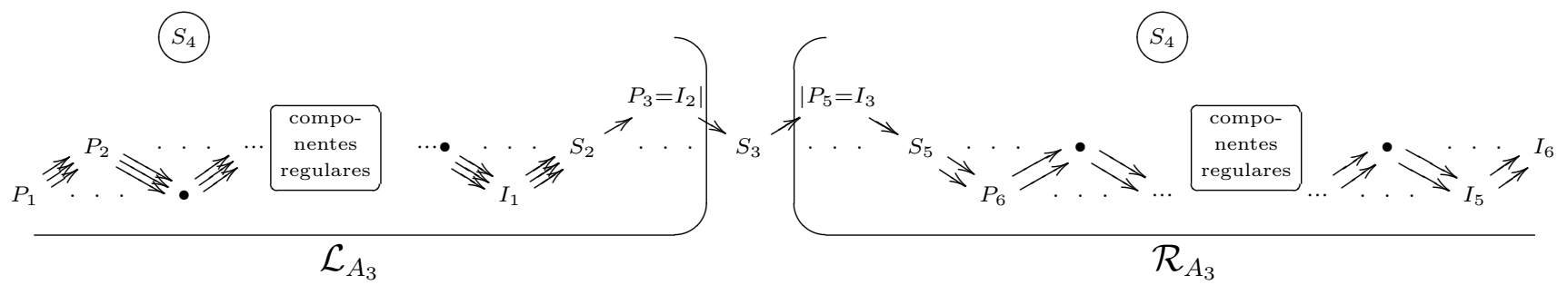

Então,

- $A_{3}$ é laura, pois (ind $\left.A_{2}\right) \backslash\left(\mathcal{L}_{A_{2}} \cup \mathcal{R}_{A_{2}}\right)=\left\{S_{3}\right\}$ é finita.

- $A_{3}$ não é colada à esquerda, pois ind $A_{3} \backslash \mathcal{R}_{A_{3}}$ não é finita.

- $A_{3}$ não é colada à direita, pois ind $A_{3} \backslash \mathcal{L}_{A_{3}}$ não é finita.

- $A_{3}$ não é shod, pois pois $S_{3} \notin \mathcal{L}_{A_{3}} \cup \mathcal{R}_{A_{3}}$.

- $A_{3}$ não é quase inclinada, pois não é shod.

- $A_{3}$ é fracamente shod, pois se $M \notin \mathcal{L}_{A_{3}}$ e $N \notin \mathcal{R}_{A_{3}}$ com $M \rightsquigarrow N$, então $M=N=S_{3}$. 
$A_{4}$ :

Consideremos $Q_{4}$ o ideal de $B$ gerado por $\alpha_{1}, \alpha_{2}, \beta_{1}$ e $\beta_{2}$. Temos que $B$ uma extensão cindida de $A_{4}=B / Q_{4}$ pelo nilpotente $Q_{4}$. Além disso, $A_{4}$ é dada pela aljava relações herdadas de $B$. Um esquema para a aljava de Auslander-Reiten dessa álgebra é:

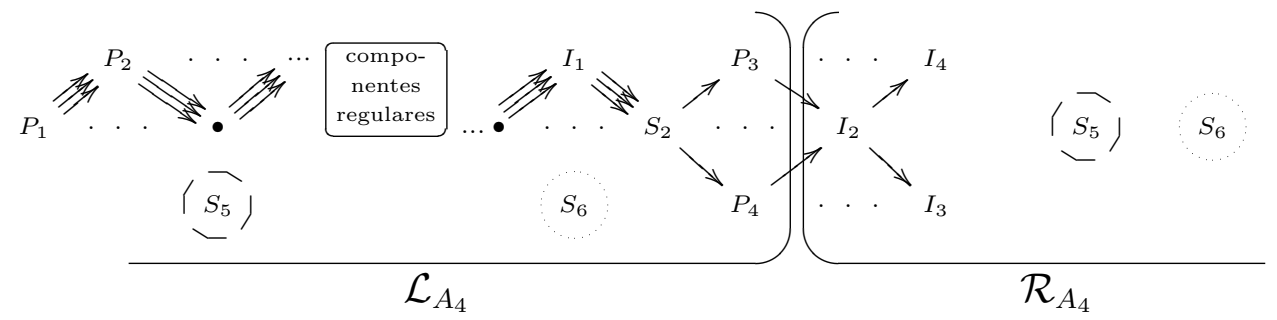

Então,

- $A_{4}$ é laura, pois (ind $\left.A_{4}\right) \backslash\left(\mathcal{L}_{A_{4}} \cup \mathcal{R}_{A_{4}}\right)=\emptyset$

- $A_{4}$ não é colada à esquerda, pois ind $A_{4} \backslash \mathcal{R}_{A_{4}}$ não é finita.

- $A_{4}$ é colada à direita, pois (ind $\left.A_{4}\right) \backslash \mathcal{L}_{A_{4}}=\left\{I_{2}, I_{3}, I_{4}\right\}$ é finita.

- $A_{4}$ é shod, pois ind $A_{4}=\mathcal{L}_{A_{4}} \cup \mathcal{R}_{A_{4}}$.

- $A_{4}$ é fracamente shod pois é shod.

- $A_{4}$ é quase inclinada, pois $P_{1}, P_{2}, P_{3}, P_{4}, P_{5}=S_{5}, P_{6}=S_{6} \in \mathcal{L}_{A_{4}}$. 


\section{$2^{\circ}$ Exemplo.}

Veremos agora que a recíproca do Teorema 5.4 não é verdadeira.

Seja $B=\frac{k \Delta}{\mathcal{I}}$ onde $\Delta$ é a aljava $1 \underset{\beta_{1}}{\stackrel{\alpha_{1}}{\leftarrow}} 2 \underset{\beta_{2}}{\stackrel{\alpha_{2}}{\leftarrow}} 3 \underset{\beta_{3}}{\stackrel{\alpha_{3}}{\leftarrow}} 4$ e $\mathcal{I}$ é o ideal gerado por $\alpha_{i} \alpha_{i-1}, \beta_{i} \beta_{i-1}, \alpha_{i} \beta_{i-1}$ e $\beta_{i} \alpha_{i-1}$ para $i=2,3$. Um esquema para a aljava de Auslander-Reiten de $B$ é

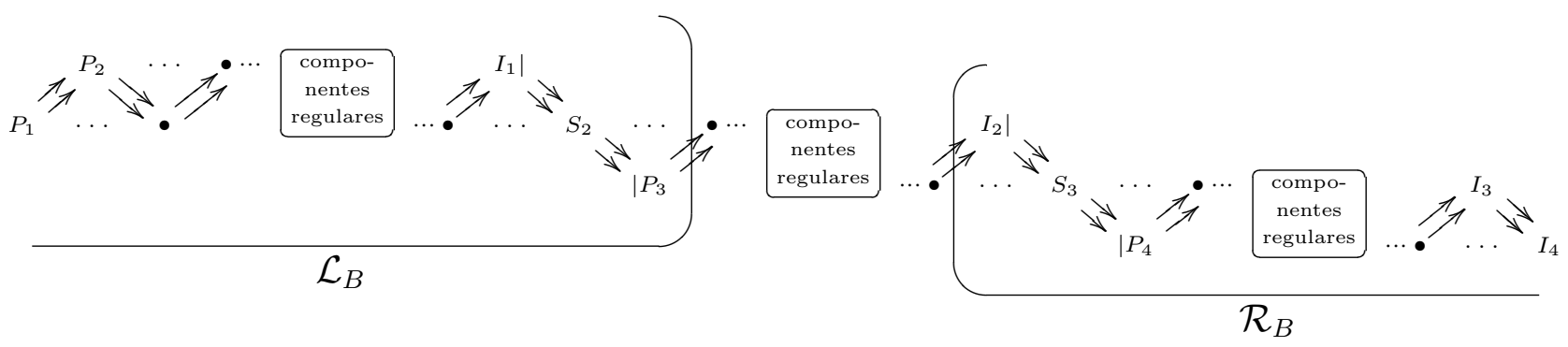

Nesse caso, $\mathcal{L}_{B} \cup \mathcal{R}_{B}$ não é cofinita em ind $B$, portanto $B$ não é uma álgebra laura.

Consideremos agora o ideal $Q$ de $B$ gerado pelas classes das flechas $\alpha_{3}$ e $\beta_{3}$. Então, $B$ é uma extensão cindida de $A=\frac{B}{Q}$ pelo ideal $Q$. A álgebra $A$ é dada pela aljava $1 \underset{\beta_{1}}{\stackrel{\alpha_{1}}{\leftarrow}} 2 \underset{\beta_{2}}{\stackrel{\alpha_{2}}{\leftarrow}} 3 \quad 4$ com as relações herdadas de $B$. Um esquema para a aljava de Auslander-Reiten de $A$ é

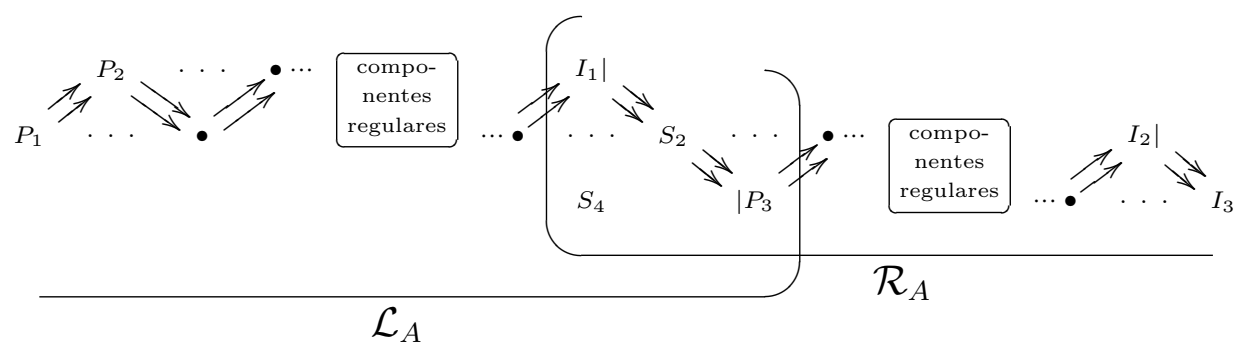

A álgebra $A$ é laura, pois ind $A \backslash\left(\mathcal{L}_{A} \cup \mathcal{R}_{A}\right)=\emptyset$. 


\section{$3^{\circ}$ Exemplo.}

Veremos agora que a recíproca do Teorema 5.13 não é verdadeira.

Seja $B=\frac{k \Delta}{\mathcal{I}}$ onde $\Delta$ é a aljava ${\underset{\beta}{\beta_{1}}}_{2}^{\alpha_{1}} \underset{\beta_{2}}{\stackrel{\alpha_{2}}{\leftarrow}} 3$ e $\mathcal{I}$ é o ideal gerado por $\alpha_{2} \alpha_{1}, \beta_{2} \beta_{1}, \alpha_{2} \beta_{1}$ e $\beta_{2} \alpha_{1}$. A aljava de Auslander-Reiten de $B$ é a mesma da álgebra $A$ do exemplo anterior, exceto pelo $S_{4}$ que nesse caso não existe. Portanto, $B$ não é uma álgebra disfarçada, pois não é colada à esquerda por exemplo.

Consideremos o ideal $Q$ de $B$ gerado pelas flechas $\alpha_{2}$ e $\beta_{2}$. Então, $B$ é uma extensão cindida de $A=\frac{B}{Q}$ pelo ideal $Q$. A álgebra $A$ é dada pela aljava $1 \underset{\beta_{1}}{\stackrel{\alpha_{1}}{\leftarrow}} 2 \quad 3$ e portanto um esquema da aljava de Auslander-Reiten para $A$ é

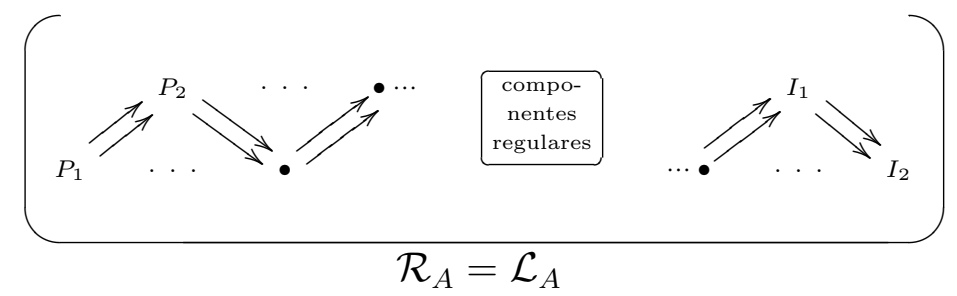

Então $A$ é disfarçada, uma vez que ind $A \backslash \mathcal{R}_{A}=\emptyset=$ ind $A \backslash \mathcal{L}_{A}$. 


\section{Apêndice A}

\section{Aljava de Auslander-Reiten}

Sejam $k$ um corpo algebricamente fechado e $C$ uma $k$-álgebra de dimensão finita. A essa álgebra associamos uma aljava, cujos vértices correspondem aos objetos da categoria ind $C$ e as flechas correspondem aos chamados morfismos irredutíveis. Por isso, começaremos definindo esses morfismos.

A construção da aljava de Auslander-Reiten não será discutida. Serão salientadas apenas algumas características dessa aljava.

As demonstrações e mais detalhes sobre esse assunto podem ser encontradas em, por exemplo, [8], [11] e [13].

Definição A.1 Sejam $M$ e $N$ dois $C$-módulos em mod $C$. Um morfismo $f \in \operatorname{Hom}_{C}(M, N)$ é dito irredutível se:

(i) f não é monomorfismo que cinde e nem epimorfismo que cinde;

(ii) se $f=g h$ então, ou $g$ é um epimorfismo que cinde, ou $f$ é um monomorfismo que cinde.

Exemplo A.1 Se P é um C-módulo projetivo indecomponível não simples, então a inclusão $\iota: \operatorname{rad} P \hookrightarrow P$ é um morfismo irredutivel.

Sejam $M$ e $N$ dois $C$-módulos em ind $C$. Denotamos por $\operatorname{rad}_{C}(M, N)$ o $k$-espaço vetorial dos morfismos de $M$ para $N$ que não são isomorfismos. Um morfismo $f: M \rightarrow N$ é irredutível se, e somente se, $f \in \operatorname{rad}_{C}(M, N) \backslash \operatorname{rad}_{C}^{2}(M, N)$. O $k$-espaço vetorial $\operatorname{Irr}(M, N)=\frac{\operatorname{rad}_{C}(M, N)}{\operatorname{rad}_{C}^{2}(M, N)}$ é chamado de espaço dos morfismos irredutíveis. 
Definição A.2 Seja $C$ uma k-álgebra de dimensão finita. A aljava de Auslander-Reiten de $C$, denotada por $\Gamma_{C}$ é a aljava definida por:

(i) os vértices estão em correspondência biunívoca com as classes de isomorfismos dos C-módulos indecomponíveis;

(ii) o número de flechas de $[M]$ para $[N]$ é igual a $\operatorname{dim}_{k} \operatorname{Irr}(M, N)$.

Decorre, diretamente da definição, que se $C$ é de tipo de representação infinito então $\Gamma_{C}$ tem infinitos vértices. Nesse caso, $\Gamma_{C}$ pode ter infinitas componentes conexas.

\section{Sequências de Auslander-Reiten}

Uma sequência exata curta de $C$-módulos que não cinde, $0 \rightarrow N \rightarrow E \rightarrow M \rightarrow 0$, é uma sequência de Auslander-Reiten (A-R) se:

(i) $M$ e $N$ são indecomponíveis;

(ii) dados $X \in \bmod C$ e $h: X \rightarrow M$ um morfismo que não seja um epimorfismo que cinde, existe $\bar{h}: X \rightarrow E$ tal que $g \bar{h}=h$.

Podemos trocar (ii) pela condição equivalente:

(iii) dados $X \in \bmod C$ e $h: N \rightarrow X$ um morfismo que não seja um monomorfismo que cinde, existe $\bar{h}: E \rightarrow X$ tal que $\bar{h} f=h$.

Proposição A.1 (Existência e unicidade) Sejam $C$ uma R-álgebra de Artin e $M$ um $C$-módulo indecomponivel.

1. Se $M$ não for projetivo, então existe e é única a sequência de A-R terminando em $M$ :

$$
0 \rightarrow N \rightarrow E \rightarrow M \rightarrow 0
$$

Além disso, $N \cong \tau M$, onde $\tau=D \operatorname{Tr}$ como definido no Capitulo 4 . 
2. Se $M$ não for injetivo, então existe e é única a sequência de $A-R$ começando em $N$ :

$$
0 \rightarrow N \rightarrow E \rightarrow M \rightarrow 0
$$

Além disso, $M \cong \tau^{-1} N$, onde $\tau^{-1}=\operatorname{Tr} D$ como definido no Capítulo 4 .

Podemos escrever uma sequência de A-R da seguinte forma

$$
0 \rightarrow N \stackrel{f}{\rightarrow} \bigoplus_{i=1}^{n} E_{i}^{t_{i}} \stackrel{g}{\rightarrow} M \rightarrow 0
$$

com cada $E_{i}$ indecomponível, $f=\left[\begin{array}{c}f_{1} \\ \vdots \\ f_{n}\end{array}\right]$ onde $f_{i}=\left[\begin{array}{c}f_{i 1} \\ \vdots \\ f_{i t_{i}}\end{array}\right]: N \rightarrow E_{i}^{t_{i}}$ e $g=\left[\begin{array}{lll}g_{1} & \cdots & g_{n}\end{array}\right]$ onde $g_{i}:\left[\begin{array}{lll}g_{i 1} & \cdots & g_{i t_{i}}\end{array}\right]: E_{i}^{t_{i}} \rightarrow M$. Nesse caso, cada $f_{i j}$ e cada $g_{i j}$ é um morfismo irredutível.

Se $M$ é um $C$-módulo indecomponível e não projetivo, então o número de flechas que saem de $[\tau M]$ é igual ao número de flechas que chegam em $[M]$. Por outro lado, se $M$ é um $C$-módulo indecomponível e não injetivo então o número de flechas que saem de $[M]$ é igual ao número de flechas que chegam em $\left[\tau^{-1} M\right]$.

$\mathrm{Na}$ aljava de A-R tal sequência corresponderia a um diagrama da forma:

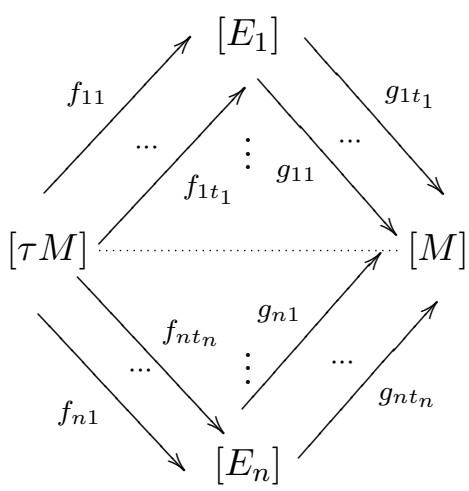

Exemplo A.2 Seja $k$ um corpo algebricamente fechado, $\Delta$ a aljava $1 \stackrel{\alpha}{\leftarrow} 2 \stackrel{\beta}{\leftarrow} 3, \mathcal{I}$ o ideal de $k \Delta$ 
gerado por $\beta \alpha$. A aljava de $A-R$ de $\frac{k \Delta}{\mathcal{I}}$ tem a seguinte forma:

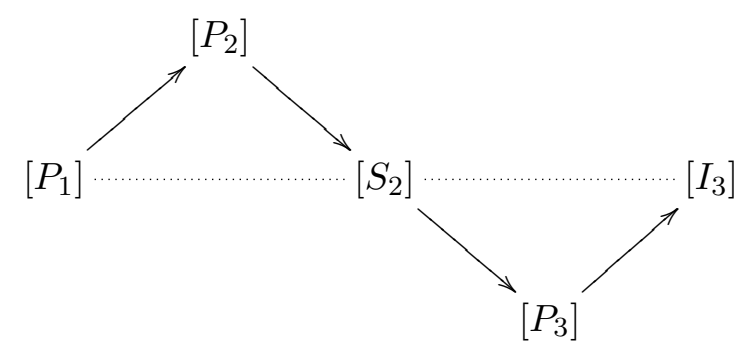

Em geral, escreveremos apenas $M$ para representar $[M]$. Por convenção as flechas sempre estão da esquerda para direita.

Vejamos qual é a relação de um morfismo qualquer entre $C$-módulos indecomponíveis e os morfismos irredutíveis.

Denotemos por $\operatorname{rad}^{\infty}(M, N):=\bigcap_{n \in \mathbb{N}} \operatorname{rad}^{n}(M, N)$. Uma componente conexa $\hat{\Gamma}$ de $\Gamma_{C}$ é dita standard generalizada se $\operatorname{rad}^{\infty}(M, N)=0$ para todos $M, N \in \hat{\Gamma}$.

Uma propriedade importante da aljava de A-R é que se $M$ e $N$ são $C$-módulos indecomponíveis tais que $M$ "aparece" à direita de $N$ na aljava, então $\operatorname{Hom}_{C}(M, N)=0$. Por isso para encontrar os predecessores (em ind $C$ ) de um $C$-módulo indecomponível, basta olhar os $C$-módulos que ficam à sua esquerda na aljava.

Proposição A.2 Seja $\hat{\Gamma}$ uma componente standard generalizada de $\Gamma_{C}$ e $M, N \in \hat{\Gamma}$. Se $f \in$ $\operatorname{Hom}_{C}(M, N)$ é não nulo e não isomorfismo então f é soma de compostas de morfismos irredutiveis.

Em particular, para uma álgebra de tipo de representação finito vale:

Corolário A.3 Seja $C$ uma álgebra de tipo de representação finito e $M$ e $N$ dois $C$-módulos indecomponíveis. Então, se $f \in \operatorname{Hom}_{C}(M, N)$ é não nulo e não isomorfismo então $f$ é soma de compostas de morfismos irredutiveis. 


\section{Referências Bibliográficas}

[1] I. Assem, Algèbres et modules, Presses de l'Université d'Ottawa, Canada, 1997.

[2] I. Assem and F. U. Coelho, Glueings of tilted algebras, Journal of Pure and Applied Algebra 96 (1994), 225-243.

[3] _ _ Two-sided gluings of tilted algebras, Journal of Algebra 269 (2003), 456-479.

[4] _ Endomorphism algebras of projective modules over laura algebras, Journal of Algebra and Its Applications 3 (2004), no. 1, 49-60.

[5] I. Assem, F. U. Coelho, M. A. Lanzilotta, D. Smith, and S. Trepode, Algebras determined by their left and right parts, Algebraic Structures and Their Representations, Cont. Math. 376 (2005), 13-47.

[6] I. Assem, F. U. Coelho, and S. Trepode, The bound quiver of a split extension, aceito para publicação no Journal of Algebra and its Applications.

[7] I. Assem and N. Marmaridis, Tilting modules over split-by-nilpotent extensions, Comm. Algebra 26 (1998), 1547-1555.

[8] I. Assem, D. Simson, and A. Skowronski, Elements of the representation theory of associative algebras, vol. 1.

[9] I. Assem and D. Zacharia, Full embeddings of almost split sequences over split-by-nilpotent extensions, Colloquium Mathematicum 81 (1999), 21-31.

[10] _ On split-by-nilpotent extensions, Colloquium Mathematicum 98 (2003), 259-275.

[11] M. Auslander, I. Reiten, and S. O. Smal $\phi$, Representation theory of artin algebras, Cambridge Stud. Adv. Math. 36, Cambridge University Press, Cambridge, 1995.

[12] H. Cartan and S. Eilenberg, Homological algebra, Princeton University Press, New Jersey, 1956.

[13] F. U. Coelho, Uma introdução à teoria de representações de álgebras (minicurso), Atas da XII Escola de Álgebra, Diamantina (1992), 60p. 
[14] F. U. Coelho and M. A. Lanzilotta, Algebras with small homological dimension, Manuscripta Math 100 (1999), 1-11.

[15] _ Weakly shod algebras, Journal of Algebra 265 (2003), 379-403.

[16] D. Happel, I. Reiten, and S. O. Smal $\phi$, Tilting in abelian categories and quasitilted algebras, Mem. Amer. Math. Soc. 575 (1996). 


\title{
Índice Remissivo
}

\author{
álgebra \\ colada \\ à direita, 73 \\ à esquerda, 73 \\ de caminhos, 21 \\ disfarçada, 76 \\ fracamente shod, 74 \\ hereditária, 67 \\ laura, 73 \\ oposta, 9 \\ quase inclinada, 75 \\ shod, 67,75 \\ parte esquerda, 70 \\ predecessor, 69 \\ retração, 6 \\ secção, 6 \\ subcategoria \\ cofinita, 72 \\ finita, 72 \\ sucessor, 69 \\ teorema \\ da adjunção, 20 \\ aljava de Auslander-Reiten, 86 \\ apresentação de uma álgebra, 24, 40 \\ conjunto minimal de geradores, 42 \\ epimorfismo cindido, 11 \\ extensão cindida por nilpotente, 27 \\ funtor \\ $C$-dual, 55 \\ de mudança de anéis, 30 \\ de Nakayama, 56 \\ dual, 15 \\ transladado de Auslander-Reiten, 56 \\ transladado de Auslander-Reiten inverso, 56 \\ transposição, 56 \\ monomorfismo cindido, 11 \\ parte direita, 70
}

